\title{
Compendium of Benchmark Neutron Fields for Reactor Dosimetry
}

\section{Standard Neutron Field Entries}

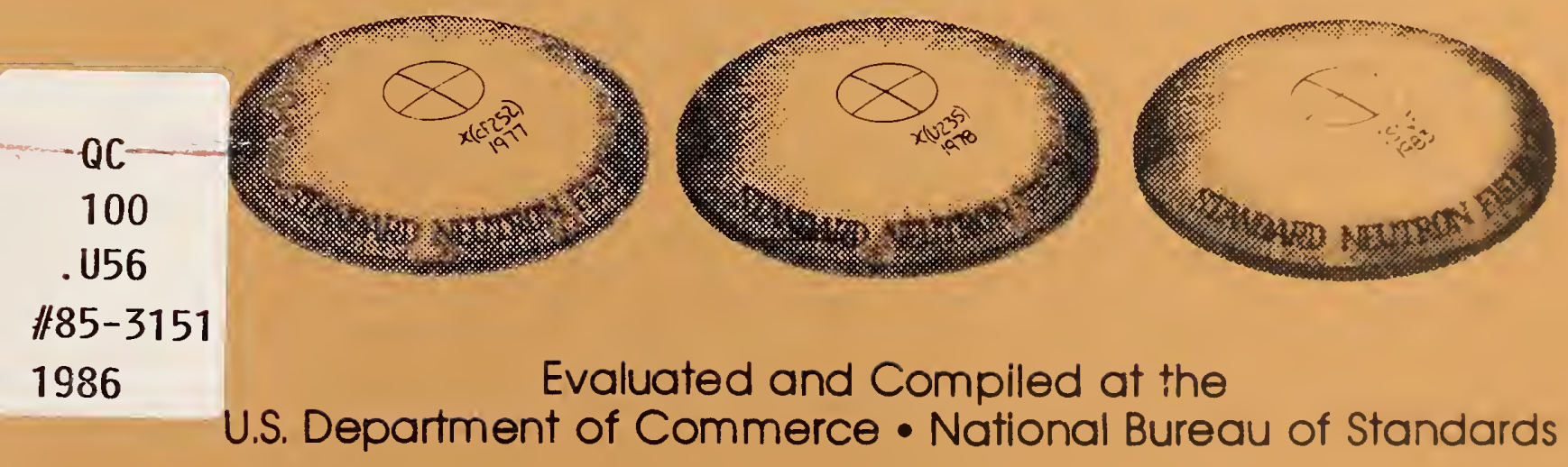


NBSIR 85-3151

COMPENDIUM OF

BENCHMARK NEUTRON FIELDS

FOR REACTOR DOSIMETRY

Standard Neutron Field Entries

James A. Grundl

Charles M. Eisenhauer

U.S. DEPARTMENT OF COMMERCE

National Bureau of Standards

Center for Radiation Research

Gaithersburg, MD 20899

January 1986

Prepared in cooperation with

U.S. Nuclear Regulatory Commission

Washington, DC 20555

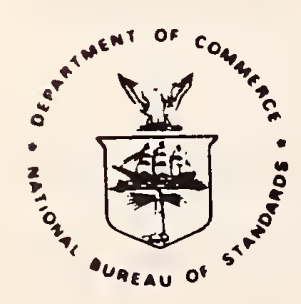

U.S. DEPARTMENT OF COMMERCE, Malcolm Baldrige, Secretary NATIONAL BUREAU OF STANDARDS, Ernest Ambler, Director 



\section{PREFACE}

Neutron dosimetry for nuclear technol ogy is a measurement discipl ine long on experience and short on major advances. Called upon to establ ish neutron exposure parameters in the most diverse kinds of radiation environments and over a wide range of neutron fluences, the basic techniques employed have changed little since early investigations were undertaken to monitor property changes in materials exposed to neutrons from fission chain reacting systems.

Small passive integral detectors, for the most part activation foils, are the backbone of measurement. They respond to neutrons over a wide energy range, often limited by a characteristic reaction threshold. The distinctive features of energy dependence among the various types of detectors form an unavoidably complex measurement base for neutron exposure estimates. An assortment of calculational methods, some of trivial sophistication and others of great ingenuity, has been used to extract rel evant neutron exposure parameters from these specialized integral measurements. Despite the many 1 imitations in this measurement method, passive integral detectors remain the technique of choice for neutron dosimetry because of their unmatched flexibility in application and their ability to distinguish neutrons from other types of radiation.

The inherent ambiguity of integral detector data, as well as the complexity of the underlying detector response measurements, places a special premium on measurement experience. Specifically, results from a particular set of passive neutron detectors often benefit from supporting measurements in one or 
more well understood neutron fields, called benchmarks. Neutron fields under study, which are less well understood and for which neutron exposure parameters are sought, will always profit from measurements in these benchmarks even when the benchmark and study fields are not well matched. Nevertheless, similarity of the fields is a benefit and for this purpose a variety of neutron fields have been developed, or pressed into service, in order to improve the accuracy of neutron dosimetry measurement techniques.

The present version of the Compendium of Benchmark Neutron Fields for Reactor Dosimetry is a revision of the original which was distributed in 1978 . It is devoted to three benchmarks called Standard Neutron Fields because they are simple, well characterized, and remain available at relatively permanent installations. The Compendium includes a detailed description of each field, its availability for calibration, calculated detector response parameters (cross sections and energy response ranges), and a set of observed reaction cross sections based on an evaluation of experimental data published before July, 1985. Neutron spectrum and integral cross section errors are included for each standard field and, at the expense of some unwelcome complexity, explained.

For purposes of clarity, space is al so devoted to neutron reaction rate formulations, error propagation, and necessary distinctions between measured and calculated detector response parameters. These formulations are included because there is no consensus in these matters among neutron dosimetrists. No attempt is intended to replace or synthesize the well worn and sometimes isolated methods by which dosimetry data is interpreted. 


\section{TABLE OF CONTENTS}

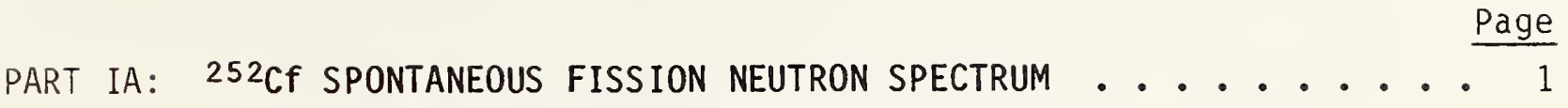

1. IDENTIF ICATIONS . . . . . . . . . . . . . . . . . 3

2. SUMMARY INFORMATION . . . . . . . . . . . . 4

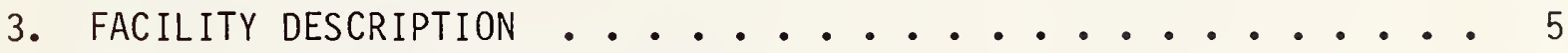

4. NEUTRON FIELD ChARACTERIZATION . . . . . . . . . . . . 8

5. INTEGRAL DETECTOR RESPONSE . . . . . . . . . . . . . . 12

6. LIST OF TABLES . . . . . . . . . . . . . . . 24

7. FIGURES . . . . . . . . . . . . . 39

PART IB: $235 U$ THERMAL-NEUTRON-INDUCED FISSION NEUTRON SPECTRUM — • • • 41

1. IDENTIFICATIONS . . . . . . . . . . . . . . 43

2. SUMMARY INFORMATION . . . . . . . . . . . . 44

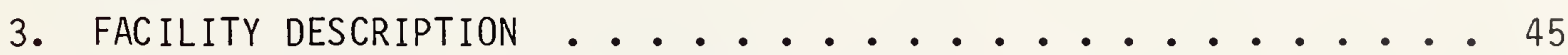

4. NEUTRON FIELD CHARACTERIZATION . . . . . . . . . . . . 49

5. INTEGRAL DETECTOR RESPONSE . . . . . . . . . . . . . 53

6. LIST OF TABLES • • • • • • • • • • • • • • • • 58

7. FIGURES . . . . . . . . . . . . . . . . . 70

PART IIA: INTERMEDIATE-ENERGY STANDARD NEUTRON FIELDS (ISNF)

WITH THICK ${ }^{10}{ }_{B}$ SHELL . . . . . . . . . . . . . 73

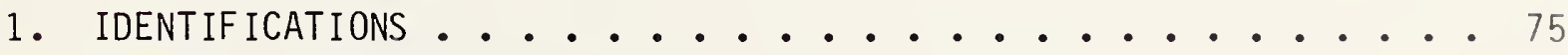

2. SUMMARY INFORMATION . . . . . . . . . . . . . . . . 77

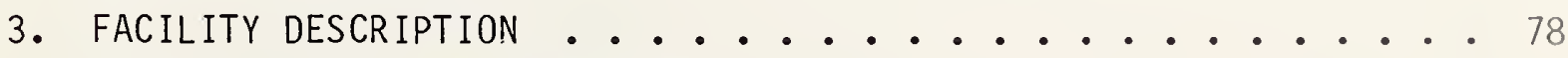

4. NEUTRON FIELD CHARACTERIZATION . • . . . . . . . . . . . 81

5. INTEGRAL DETECTOR RESPONSE • • • • • • • • • . • . . 96

6. LIST OF TABLES •. • . . . . . . . . . . . . . . 101

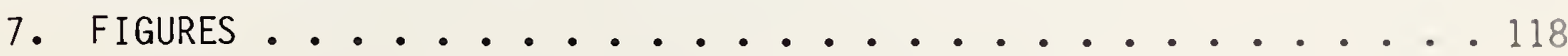

REFERENCES . . . . . . . . . . . . . . . . 125 



Part IA: ${ }^{252} \mathrm{Cf}$ Spontaneous Fission Neutron Spectrum 


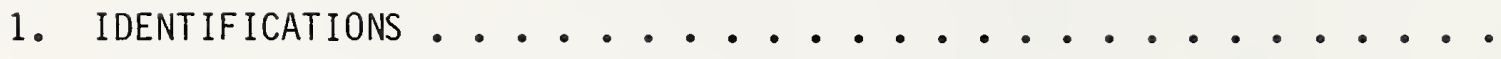

1.a. Title, Classification

1.b. Designation

1.c. Entry Dates and Revisions

1.d. Generic Description

1.e. Facility Locations

1.f. Contacts for Information

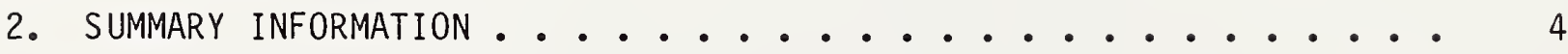

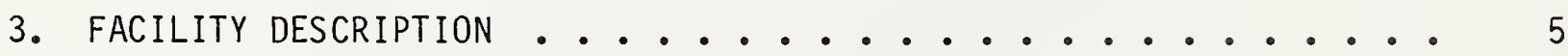

3.a. Configuration and Characteristics

(location, source, scattering corrections)

3.b. Irradiation Procedures

(experimental arrangements, neutron fluence)

3.c. Specification For Transport Calculations

4. NEUTRON FIELD CHARACTERIZATION .............

4.a. Neutron Fluence

4.b. Neutron Spectrum

( $f$ luence rate, compensated beam geometry)

4.b.1. Calculation

4.b.2. Measurement

4.b.3. Evaluated Spectrum

(multigroup format, spectrum errors)

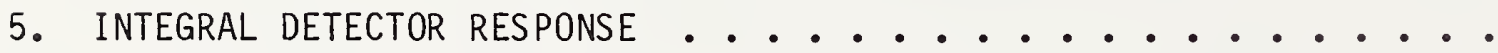

5.a. Calculated Reaction Probabilities

(formulation, truncation, spectrum fraction)

5.a.1. Spectrum Response Table

(cross sections, spectrum fraction, response range)

5.a.2. Spectral Indexes

5.a.3. Error Propagation

5.b. Measured Reaction Probabilities

(formulation, spectral indexes, neutron fluence transfer)

5.c. Measured Cross Sections and Calculated-to-Observed Ratios

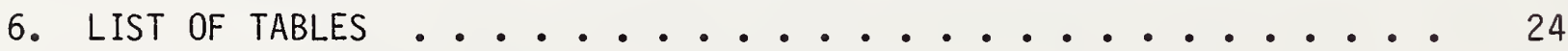

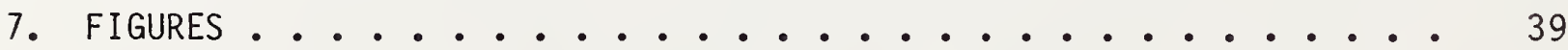




\section{IDENTIFICATIONS}

1.a. NEUTRON FIELD: ${ }^{252} \mathrm{CF}$ SPONTANEOUS FISSION SPECTRUM CLASS IFICATION: STANDARD NEUTRON FIELD

1.b. DESIGNATION: XCF-5-NT

NBS 1975 evaluation of documented differential spectrum measurements $[\mathrm{Gr} 75 \mathrm{~b}]$ and $[\mathrm{Gr} 75 \mathrm{c}]$

1.c. ENTRY DATE: May, 1978

REVISIONS: July, 1985

July, 1983

\section{1.d. GENERIC DESCRIPTION}

A standard ${ }^{252} \mathrm{Cf}$ neutron field consists of neutrons from the spontaneous fission of ${ }^{252} \mathrm{Cf}$ with little or no energy degradation from collided fission or other background neutrons. The median energy of the spectrum is $1.68 \mathrm{MeV}$, with $98 \%$ of the neutrons between $0.1 \mathrm{MeV}$ and $8 \mathrm{MeV}$. Neutron fluence rates in the range of $10^{7} \mathrm{n} /\left(\mathrm{cm}^{2} \mathrm{~s}\right)$ are obtained in isolated environments near small, intense ${ }^{252} \mathrm{Cf}$ fission sources. Neutron fluences are established in terms of neutron source strength, irradiation time, and source-detector distance; no microscopic nuclear data or irradiation monitor are required. Certified freefield fluences of up to $10^{13} \mathrm{n} / \mathrm{cm}^{2}$ may be obtained with uncertainties as low as $\pm 1.3 \%(1 \sigma)$.

Measurements of the ${ }^{252} \mathrm{Cf}$ fission neutron spectrum and its close relative the $235 \mathrm{U}$ fission spectrum are extensive and well-documented. These two standard neutron fields, therefore, are much better known than any other benchmark employed for reactor dosimetry calibration. Moreover, in the energy range above 2 MeV many neutron fields in and around test and power reactors have fission-spectrum-like components. 
1.e. FACILITY LOCATIONS:

National Bureau of Standards (NBS)

Center for Radiation Research

Gaithersburg, Maryl and 20899

U.S.A.

Contact: Dale McGarry or James Grundl

Phone: 301-921-2767

Physikalisch-Technische

Bundesanstalt (PTB)

33 Braunschweig

Federal Republic of Germany

Contact: Wolfgang G. Alberts

SEFOR Calibration Facility

University of Arkansas

Fayetteville, AR 72701

U.S.A.

Contact: Leon West

Phone: 501-575-3449

1.f. CONTACT FOR INFORMATION:

Dale McGarry or George Lamaze

Center for Radiation Research

National Bureau of Standards

Gaithersburg, Maryland 20899

U.S.A.

Phone: 301-921-2767

2. SUMMARY INFORMATION

2.a. AVAILABLE FISSION NEUTRON EXPOSURES (NBS ONLY)

- fluence rate $\left(5 \mathrm{~cm}\right.$ from source) $\sim 2 \times 10^{7} \mathrm{n} /\left(\mathrm{cm}^{2} \mathrm{~s}\right)$

- nominal maximum fluence $\quad 1 \times 10^{13} \mathrm{n} / \mathrm{cm}^{2}$

- accuracy of free-field fluence $(10) \pm 1.3 \%$

2.b. CORRECTIONS FOR NEUTRON SCATTERING AT 5 CM POSITION (NBS ONLY)

- Net perturbation for threshold detector reaction rates: < $(1.0 \pm 0.8) \%$

- Net perturbation for 235 fission reaction rate: $(1.2 \pm 0.4) \%$ 


\section{FACILITY DESCRIPTION}

Fission neutron irradiation facilities employing intense ${ }^{252} \mathrm{Cf}$ spontaneous fission sources exist at the National Bureau of Standards, the SEFOR Cal ibration Center in Arkansas, and the PTB Standards Laboratory in the Federal Republic of Germany. Only the NBS facility which is in general use for neutron detector calibrations and cross section measurements will be described in detail. Distinguishing characteristics of other irradiation facilities may be ascertained from the 1 iterature ([Ma79a], [A1 75a], [A1 75b], [Gr75a], $[\mathrm{Br} 81 \mathrm{a}],[\mathrm{Br} 81 \mathrm{~b}])$.

\section{3.a. CONFIGURATION AND CHARACTERISTICS [Gr77a], [La82a]}

The two irradiation locations at NBS are distinguished by the degree to which the neutron source is isolated from environmental neutron return: Location $A$ : Large room with thick walls and open ceiling. Source 2.2 meters above floor, nearest wall 4.1 meters.

Location B: Corner area of room with thin walls and ceiling. Source 2.8 meters above floor.

At both locations a light-weight source-detector assembly is available for irradiation of passive and active neutron detectors. The source-detector assembly with two active fission chambers mounted on a single ax is is shown in Fig. X-1. Alternatively, simultaneous exposure in pairs may be performed along three separate axes.

The cal ifornium source capsule shown in Fig. $X-2$, is made up of a disk-shaped ${ }^{252} \mathrm{Cf}$ deposit in an aluminum pellet encapsulated in a thin-walled stainless steel cylinder. The position of the cal ifornium deposit relative to the 
capsule surface is known to $\pm 0.5 \mathrm{~mm}$ based on constraints of fabrication and $x$-ray photographs. Newer capsule designs feature a short attachment stem in place of the first few $\mathrm{cm}$ of the source guide tube; the ${ }^{252} \mathrm{Cf}$ deposit enclosure is unchanged.

Neutron field parameters and error estimates for a nominal $5 \mathrm{~cm}$ source-todetector distance are given in Table $X-1$. The source strength uncertainty of $\pm 1.1 \%$ dominates the composite error of $\pm 1.3 \%$ for the free-field fission neutron fluence. The irradiation geometry shown in Fig. X-1 is termed compensated-beam geometry and refers to the experimental practice of placing detectors of similar sensitivity in pairs on opposite sides of the source, and nearly equidistant from it. The geometric mean of the responses of the two detectors in this case can be expressed in terms of a mean neutron fluence which is a function of detector separation with very little dependence on source position. The source and the detectors may be rotated during the irradiation to further ensure proper spatial averaging of the neutron fluence and scattering at the detectors.

Scattering Corrections. Neutron fluence perturbations in location B attributable to room return, scattering in the source capsule and in support structures are given in Table $X-2$. Corresponding detector perturbations are listed for a threshold, and a low-energy response neutron detector. The roomreturn component is estimated from calculation matched to response-versusdistance measurements in which the source is moved while the detectors are kept at their normal $5 \mathrm{~cm}$ position [Li84a]. For detectors with low-energy response, a large cadmium basket is placed around the source-detector assembly to absorb room-return thermal neutrons. The fission neutron return from this basket is less than $0.1 \%$. 
Three types of calculations are employed for estimating free-field neutron fluences: (1) discrete ordinates calculation of a spherical cavity in concrete to obtain the albedo from boundaries; (2) geometry and simple energy degradation factors calculated for single-scatter events in individual pieces to obtain corrections for scattering in source capsule and support structures; and (3) calculations based on published analytical formulations involving a simple scattering kernel to obtain estimates of air scattering. Air scatter contributions are less than $0.1 \%$ for source-detector distances of $5 \mathrm{~cm}$ and $<0.5 \%$ for distances up to $15 \mathrm{~cm}$.

The degree of difficulty in correcting a free-field neutron fluence for scattering in a detector depends upon the mass, arrangement, and material of the detector. The NBS has codes available which provide correction factors for isotropic scattering of neutrons in lightly constructed detectors with cylindrical symmetry. More extensive Monte-Carlo calculations have been carried out for special detectors, specifically the NBS double fission chamber. Multiple scattering in more massive detectors are difficult to estimate and often require auxiliary experiments. Generally, isolated ${ }^{252} \mathrm{Cf}$ fission neutron fields are most appropriate for accurate, uncluttered exposures with light weight detectors.

\section{3.b. IRRADIATION PROCEDURES}

Detector pairs of similar sensitivity are hung from the mounting ring on opposite sides and equidistant from the source as shown in Fig. X-1. Alignment and the exact distance between front faces of detectors are determined with a computer controlled digital cathetometer fitted with a piezoelectric sensor. After the separation distance is established, the mounting ring is 
placed in the irradiation facility and the ${ }^{252} \mathrm{Cf}$ source is raised from the storage hole for a timed exposure. Exposure times in the range of a few minutes to a maximum of one week are available. Packages of detector pairs may be irradiated simultaneously along three separate axes. No irradiation monitor is required since californium is a natural neutron source with an emission rate governed by its nuclear decay constant (2.2\% decrease per month).

A mean free-field fission neutron fluence for detector pairs (i.e., the fluence in the absence of all neutron scattering effects) is obtained from the known ${ }^{252} \mathrm{Cf}$ neutron source strength, the detector separation distance, and the irradiation time - see Section 4a. This fluence can be specified to a maximum accuracy of $\pm 1.3 \%(1 \sigma)$.

\section{3.c. SPECIFICATION FOR NEUTRON TRANSPORT CALCULATION}

Neutron transport calculations are employed for scattering corrections. Material specifications for the source are indicated in Section 3a and in Fig. $X-2$.

\section{NEUTRON FIELD CHARACTERIZATION}

The energy spectrum of ${ }^{252} \mathrm{Cf}$ spontaneous fission neutrons is similar to that of $235 \mathrm{U}$ and other fissionable materials. As such it provides a spectrum of neutrons characteristic of the driving source for most of nuclear energy. Because fission neutron spectra are similar and have been evaluated at the same time, the ${ }^{252} \mathrm{Cf}$ spectrum is specified here together with the $235 \mathrm{U}$ spectrum. A concise description of fission neutron spectra may be given in terms of a broad energy range, 


$$
\begin{array}{lcl}
\text { lower bound } & \text { median } & \text { upper bound } \\
E_{p}(p=0.99) & E_{p}(p=0.5) & E_{p}(p=0.01)
\end{array}
$$

$\begin{array}{llll}{ }^{252} \mathrm{Cf} & 0.09 \mathrm{MeV} & 1.68 \mathrm{MeV} & 7.8 \mathrm{MeV} \\ 235 \mathrm{U} & 0.08 & 1.57 & 7.2\end{array}$

\begin{tabular}{|c|c|c|c|c|c|c|}
\hline 0 & & .8 & 1.5 & 2.3 & 3.7 & 8 \\
\hline$(0.047)$ & 0.184 & 0.220 & 0.194 & 0.200 & 0.146 & 0.009 \\
\hline$(0.054)$ & 0.197 & 0.229 & 0.195 & 0.192 & 0.127 & 0.006 \\
\hline
\end{tabular}

and in a coarse seven-group display, $\phi(E) \Delta E$, as follows:

A more detailed description of the two spectra is given in Section 4.b.

Prompt fission gamma-rays and gammas from fission products contribute about equally to the total gamma-ray emission throughout the useful lifetime of the source. As indicated in Table $X-1$, the neutron-to-gamma ratio is about $1.1 \times 10^{5}\left(\mathrm{n} / \mathrm{cm}^{2} \mathrm{~s}\right)$ per $\mathrm{R} / \mathrm{h}$ at $5 \mathrm{~cm}$.

4.a. NEUTRON FLUENCE (see also Section 3.a.)

The neutron emission rate for the singly-encapsulated sources employed at NBS are $\sim 6 \times 10^{9} \mathrm{n} / \mathrm{s}$ when fabricated and decays at a rate of $2.2 \%$ per month. For a neutron fluence rate of $\sim 2 \times 10^{7} \mathrm{n} /\left(\mathrm{cm}^{2} \mathrm{~s}\right)$ at the 5 - $\mathrm{cm}$ distance, the fluence for a one week exposure is $\sim 1 \times 10^{13} \mathrm{n} / \mathrm{cm}^{2}$. Spatial gradients for uncollided fission neutrons are just those of a point source in free space: $\frac{\Delta \phi}{\phi}=\frac{2 \Delta r}{r}$. Free-field fluence accuracy and detector response perturbations for typical irradiation arrangements are discussed in Section 3.a. 
In the case of compensated-beam geometry where nearly identical detectors are placed on opposite sides of the source and nearly equidistant from it, a mean neutron fluence rate may be defined,

$$
\begin{aligned}
\langle\Phi\rangle & =\frac{S}{4 \pi(r / 2)^{2}} \\
S & =\text { source strength }(\mathrm{n} / \mathrm{s}) \\
r & =\text { distance between detectors }(\mathrm{cm}),
\end{aligned}
$$

which is proportional to the geometric mean of the detector responses per atom:

$$
\begin{aligned}
\sqrt{\frac{D_{1}}{N_{1}} \frac{D_{2}}{N_{2}}} & =a \sigma\langle\Phi\rangle \\
\text { where } D / N & =\text { detector response per atom } \\
\sigma & =\text { reaction cross section } \\
a & =\text { efficiency factor (same for both detectors). }
\end{aligned}
$$

[The exact form of the above expression departs by $<0.2 \%$ for $D_{1} / N_{1}$ within $20 \%$ of $\left.\mathrm{D}_{2} / \mathrm{N}_{2} \cdot\right]$

A small anisotropy results from the cylindrical shape of the fission source capsule. The ratio of the fluence rate averaged over all directions to the fluence rate along a direction perpendicular to the capsule ax is is 0.992 when the detectors do not subtend an angle of more than about $20^{\circ}$ at the source (see Fig. $X-1)$.

\section{4.b. NEUTRON SPECTRUM}

4.b.1. Calculation. Fission neutron spectra are most accurately determined by 1 aboratory measurement. (See, however, Refs. [Ma82b], [Ma84c] and earlier work cited therein.) 
4.b.2. Measurement $[\operatorname{Gr} 75 b],[G r 75 c]$. From 1952 to the present, welldocumented measurements of ${ }^{252} \mathrm{Cf}$ and ${ }^{235} \mathrm{U}$ fission neutron spectra have employed every perfected technique of neutron spectrometry [Wi70a]. Accordingly, fission spectra are the most widely studied fast-neutron energy distribution in existence. The large body of documented fission spectrum data was evaluated at NBS in 1975 [Gr75c]. The evaluation included an estimate of the spectrum uncertainties in multigroup format based on the spread of the various data sets. The ${ }^{252} \mathrm{Cf}$ and ${ }^{235} \mathrm{U}$ fission spectrum shapes recommended in the evaluation are based on the sixteen selected measurements listed in Table $x-3$.

4.b.3. Evaluated Spectrum. The NBS fission spectrum evaluation is chosen for this compendium. The ${ }^{252} \mathrm{Cf}$ spectrum is defined up to $20 \mathrm{MeV}$ by means of a reference Maxwelliam, $M(E)$, modified by four piecewise continuous linear segments below $6 \mathrm{MeV}$ plus one exponential segment above $6 \mathrm{MeV}$. The reference Maxwellian is for the ${ }^{252} \mathrm{Cf}$ fission spectrum is

$$
M(E)=0.667 \sqrt{E} \cdot \exp (-1.5 E / 2.13), \quad E \text { in } M e V \text {, }
$$

with adjustment functions $\mu(E)$ as follows:

Energy Interval (MeV)

${ }_{C f}(E)$

$\begin{array}{lll}0.0-0.25 & 1+1.20 E-0.237 \\ 0.25-0.8 & 1-0.14 E+0.098 \\ 0.8-1.5 & 1+0.024 E-0.0332 \\ 1.5-6.0 & 1-0.0006 E+0.0037 \\ 6.0-20 & 1.0 \exp [-0.03(E-6.0) / 1.0]\end{array}$


The evaluated spectrum then is given by $x(E)=\mu(E) \cdot M(E)$. A 45-group tabulation of the evaluated ${ }^{252} \mathrm{Cf}$ fission spectrum is given in Table $X-4$ along with the corresponding $235 \mathrm{U}$ evaluated spectrum (see Part I-B, Section 4.b). For other energy group structures this tabulation may be interpolated as recommended in the footnote of Table $X-4$.

Spectrum uncertainties in the evaluation are based on departures of subsets of measured data from the final adjusted Maxwellian. This estimate, carried out in a seven group structure, includes both $1 \sigma$ and $2 \sigma$ errors. Results are given in Table $\mathrm{X}-5$.

\section{INTEGRAL DETECTOR RESPONSE}

Neutron dosimetry measurements with integral detectors generally benefit from the use of benchmark neutron fields to calibrate measurement techniques and to reference data interpretation methods. In order to carry out such measurement assurance procedures, and in particular for estimating uncertainties, it is necessary to distinguish between calculated and measured reaction probabilities and to identify parameters which characterize the energy response of each detector in the various spectra to which it is exposed. A formulation which meets these requirements including expressions for first-order error propagation is presented briefly in Sections 5.a. and 5.b. Tables of calculated response parameters list cross sections, energy response ranges, and spectral indexes for a number of integral detectors. An evaluation of measured reaction cross sections, discussed in Section 5.c., ends with a comparison of measured and calculated values including an error propagation based on fission spectrum uncertainties given in Table $X-5$. 


\section{5.a. CALCULATED REACTION PROBABILITIES}

The calculated reaction probability, $R_{C}$, for an integral detector is equal to the product of the neutron fluence to which it has been exposed and the spectrum-averaged reaction cross section:

$$
\begin{gathered}
\mathrm{R}_{\mathrm{C}}=\bar{\sigma} \cdot \Phi \\
\Phi=\text { total spectrum integrated neutron fluence, }\left(\mathrm{n} / \mathrm{cm}^{2}\right) \cdot \\
\bar{\sigma}=\text { spectrum-averaged reaction cross section: } \int_{0}^{\infty} \sigma(E) \psi(E) d E,
\end{gathered}
$$

where $\psi(E)$ is the neutron spectrum normalized to unity. (In many applications the lower limit of integration is $0.4 \mathrm{eV}$, the cadmium cut-off.)

The reaction probability is sometimes referred to as the total reactions per target nucleus or the time-integrated reaction rate. In this formulation all experimental quantities such as detection efficiencies, time history corrections, and neutron field perturbations are relegated to the expression for the measured reaction probability given in Eq. $(X-10)$ of Section 5.b.

Integral detector response al so may be expressed in terms of an average neutron fluence rate, $\langle\phi\rangle=\Phi / T$, where $T$ is the effective irradiation interval. In this case, the calculated quantity of interest (experimentally, the saturated specific activity for activation detectors) is the average reaction rate: $\bar{\sigma} \phi\rangle$. 
When neutron spectra extend over a large energy range, a truncated neutron fluence, $\left[\psi\left(>E_{0}\right) \cdot \Phi\right]$, may be defined where $\psi\left(>E_{0}\right)$ is the spectrum fraction above $E_{0}$. An example from materials damage dosimetry is $[\psi(>1 \mathrm{MeV}) \cdot \Phi]$, the fluence greater than 1 MeV. Similarly, for integral detectors with a restricted energy response range (e.g., threshold detectors), a cross section truncated near the lower bound of the detector response range is a measure of detector response invariance to spectrum shape:

$$
\left.\bar{\sigma}>E_{p}\right)=\frac{\int_{E_{p}}^{\infty} \sigma(E) \psi(E) d E}{\int_{E_{p}}^{\infty} \psi(E) d E}, E_{p}=\text { truncation energy. }
$$

For a fractional response $\mathrm{p}$, the truncation energy is defined by

$$
p \cdot \bar{\sigma}=\int_{E_{p}}^{\infty} \sigma(E) \psi(E) d E=\bar{\sigma}\left(>E_{p}\right) \cdot \psi\left(>E_{p}\right)
$$

where $\psi\left(>E_{p}\right)=$ the spectrum fraction above $E_{p}$

$$
\begin{aligned}
p & =\text { fraction of detector response above } E_{p} ; \\
E_{p}(p=1) & =0.4 \mathrm{eV} ; \quad E_{p}(p=0)=20 \mathrm{MeV} ; E_{p}(p=0.5)=\text { median energy. }
\end{aligned}
$$

The advantage of a truncated cross section $\bar{\sigma}\left(>E_{p}\right)$ is its independence of spectrum in the energy region where the detector does not respond.

In terms of these energy-truncated quantities the calculated reaction probability of Eq. $(X-1)$ may be written as

$$
R_{c}=\frac{\psi\left(>E_{p}\right)}{p \cdot \psi\left(>E_{0}\right)} \cdot \bar{\sigma}\left(>E_{p}\right) \cdot\left[\psi\left(>E_{0}\right) \cdot \Phi\right] .
$$


An alternative formulation of truncated fluence which deals with $\bar{\sigma}\left(>E_{0}\right)$ in place of $\bar{\sigma}\left(>E_{p}\right)$ in Eq. $(X-4)$ has been in some use in the past. The relationship between the two formulations follows directly from the definition and Eq. $(x-4)$,

$$
\begin{aligned}
\bar{\sigma}\left(>E_{0}\right) & \equiv \frac{[\text { reaction probability] }}{\left[\text { fluence above } E_{0}\right]} \\
& =\frac{\psi\left(>E_{p}\right)}{p \cdot \psi\left(>E_{0}\right)} \cdot \bar{\sigma}\left(>E_{p}\right) .
\end{aligned}
$$

5.a.1. Spectrum Response Table. Basic integral detector response parameters for ${ }^{252} \mathrm{Cf}$ fission neutrons are given in Table $\mathrm{X}-6$ (B5). Spectrum averaged cross sections (above a cadmium cut-off energy of $0.4 \mathrm{eV}$ ), listed in column 2, are followed in column 3 by cross sections truncated at $p=0.95$. The energy dependent cross sections employed are those of the ENDF/B-V dosimetry file reduced to 620 energy groups; spectrum averaging is carried out with the NBS DETAN code. Spectrum fractions for each detector, $\left[\psi\left(>E_{p}\right)\right.$, $p=0.95]$, are 1isted in column 4. Energy response characteristics given in the last three columns, are the median response energy and lower- and upperenergy bound s that include $90 \%$ of the detector response. The lower-energy bound, $E_{p}(p=0.95)$, is the truncation energy corresponding to the truncated cross section given in column 3 .

5.a.2. Spectral Indexes. Elementary spectrum indicators associated with integral detector responses are the ratios of spectrum-averaged cross sections among detector pairs with distinguishable energy response ranges. This ratio is generally called a spectral index:

$$
S_{\alpha / \beta}=\bar{\sigma}_{\alpha} / \bar{\sigma}_{\beta}
$$

where $\alpha$ and $\beta$ refer to the two detector reactions involved. 
A selected set of spectral indexes calculated for full-spectrum-averaged cross sections is given in Table $X-7(B 5)$. The corresponding set for truncated cross sections is in Table $X-8(B 5)$.

The ratio of the calculated spectral index to the observed value, $C_{\alpha / \beta}$, is the basic spectrum information available from an integral detector measurement.

$$
C_{\alpha / \beta}=\left[S_{\alpha / \beta}\right]_{C a l} /\left[S_{\alpha / \beta}\right]_{\text {obs }} \text {. }
$$

These double ratios should be established consistently both for benchmark neutron fields and for neutron fields under study. Then, by a variety of analytic means, the double ratios may be employed for (1) directly adjusting the calculated spectrum of the neutron field under study; (2) normalizing neutron transport calculations; (3) establishing spectrum consistency among the benchmark fields themselves; or (4) validating or adjusting reaction cross sections. In particular, a benchmark neutron field calibration may be established by normalizing the double ratio for a study spectrum to the corresponding double ratio for a relevant benchmark:

$$
\left[T_{\alpha / \beta}\right]_{C} \equiv\left[C_{\alpha / \beta}\right]_{\text {study }} /\left[_{\alpha / \beta}\right]_{\text {bmk }} \text {. }
$$

5.a.3. Error Propagation. Apart from the complexities of assessing correlations, expressions for estimating the uncertainty of calculated spectrum response parameters can be obtained directly from first-order statistical propagation. Four error propagation expressions for uncorrelated errors in spectrum and cross section uncertainties are included here. Others may be found in Ref. [Gr82a] along with an example of their application to reactor pressure vessel irradiation surveillance dosimetry. 
1. A full-spectrum-averaged cross section, $\bar{\sigma}$, in multigroup format is

$$
\bar{\sigma}=\sigma_{0} \cdot \sum_{i} s_{i} \psi_{i} \Delta E_{i} / \sum_{i} \psi_{i} \Delta E_{i}
$$

where $\sigma_{i} \equiv \sigma_{0} s_{i}$ represents the energy dependent reaction cross section and $\psi_{i}$ the neutron spectrum. The standard error for $\bar{\sigma}$, without covariance, is

$$
(\delta \bar{\sigma})^{2}=\sum_{\dot{i}}\left[\left(\frac{\partial \bar{\sigma}}{\partial s_{i}}\right)^{2}\left(\delta s_{i}\right)^{2}+\left(\frac{\partial \bar{\sigma}}{\partial \psi_{i}}\right)^{2}\left(\delta \psi_{j}\right)^{2}+\left(\frac{\partial \bar{\sigma}}{\partial \sigma_{0}}\right)^{2}\left(\delta \sigma_{0}\right)^{2}\right] .
$$

The fractional standard error, $\delta \bar{\sigma} / \bar{\sigma}$, follows directly:

$\left(\frac{\delta \bar{\sigma}}{\bar{\sigma}}\right)^{2}=\sum_{\mathbf{i}}\left[\frac{\sigma_{i}}{\bar{\sigma}} \psi_{\mathbf{i}} \Delta \mathrm{E}_{\mathbf{i}}\right]^{2}\left(\frac{\delta \mathrm{s}_{\mathbf{i}}}{\mathbf{s}_{\mathbf{i}}}\right)^{2}+\sum_{\mathbf{i}}\left[1-\frac{\sigma_{\mathbf{i}}}{\bar{\sigma}}\right]^{2}\left(\psi_{\mathbf{i}} \Delta \mathrm{E}_{\mathbf{i}}\right)^{2}\left(\frac{\delta \psi_{\mathbf{i}}}{\psi_{\mathbf{i}}}\right)^{2}+\left(\frac{\delta \sigma_{0}}{\sigma_{0}}\right)^{2} \cdot(\mathrm{X}-7)$

The quantities $\delta \psi_{\mathbf{j}} / \psi_{\mathbf{j}}$ and $\delta s_{\mathbf{j}} / \mathrm{s}_{\mathbf{j}}$ are the fractional errors in the normal ized neutron spectrum and the cross-section shape, respectively.

2. The fractional error for a spectral index, $S_{\alpha / \beta}=\bar{\sigma}_{\alpha} / \bar{\sigma}_{\beta}$, is similar,

$$
\begin{aligned}
& \left(\frac{\delta S_{\alpha / \beta}}{S_{\alpha / \beta}}\right)^{2}=\sum_{\mathbf{i}}\left[\left(\frac{\sigma_{\alpha \mathbf{i}}}{\sigma_{\alpha}}\right)^{2}\left(\frac{\delta s_{\alpha \mathbf{i}}}{s_{\alpha \mathbf{i}}}\right)^{2}+\left(\frac{\sigma_{\beta \mathbf{i}}}{\sigma_{\beta}}\right)^{2}\left(\frac{\delta s_{\beta \mathbf{i}}}{s_{\beta i}}\right)^{2}\right]\left(\psi_{\mathbf{i}} \Delta E_{\mathbf{i}}\right)^{2} \\
& +\sum_{\mathbf{i}}\left(\frac{\sigma_{\alpha \mathbf{i}}}{\sigma_{\alpha}}-\frac{\sigma_{\beta \mathbf{i}}}{\sigma_{\beta}}\right)^{2}\left(\psi_{\mathbf{i}} \Delta \mathrm{E}_{\mathbf{i}}\right)^{2}\left(\frac{\delta \psi_{\mathbf{i}}}{\psi_{\mathbf{j}}}\right)^{2}+\left(\frac{\delta \sigma_{0 \alpha}}{\sigma_{0 \alpha}}\right)^{2}+\left(\frac{\delta \sigma_{0 \beta}}{\sigma_{0 \beta}}\right)^{2} .
\end{aligned}
$$

3. The fractional error corresponding to a cross section ratio for one detector in two different neutron fields, $s_{b / s}=\bar{\sigma}_{b} / \bar{\sigma}_{s}$, is 


$$
\begin{aligned}
\left(\frac{\delta S_{b} / s}{S_{b} / s}\right)^{2} & =\sum_{i}\left[\psi_{b i} \frac{\sigma_{i}}{\bar{\sigma}_{b}}-\psi_{s i} \frac{\sigma_{i}}{\bar{\sigma}_{s}}\right]^{2}\left(\Delta E_{i}\right)^{2}\left(\frac{\delta s_{i}}{s_{i}}\right)^{2} \\
& +\sum_{i}\left(1-\frac{\sigma_{i}}{\bar{\sigma}_{b}}\right)^{2}\left(\psi_{i} \Delta E_{i}\right)^{2}\left(\frac{\delta \psi_{b i}}{\psi_{b i}}\right)^{2}+\sum_{i}\left(1-\frac{\sigma_{i}}{\bar{\sigma}_{s}}\right)^{2}\left(\psi_{i} \Delta E_{i}\right)^{2}\left(\frac{\delta \psi_{s i}}{\psi_{s i}}\right)^{2} .
\end{aligned}
$$

4. The truncated spectrum-averaged cross section in multigroup form is,

$$
\sigma\left(>E_{p}\right)=\sigma_{0} \sum_{i>i_{p}} s_{i} \psi_{i} \Delta E_{i} / \sum_{i>i} \psi_{i} \Delta E_{i}
$$

and the fractional error is

$$
\begin{aligned}
{\left[\frac{\delta \bar{\sigma}\left(>E_{p}\right)}{\bar{\sigma}\left(>E_{p}\right)}\right]^{2} } & =\sum_{i>i_{p}}\left[\mu_{i} \frac{\sigma_{i}}{\bar{\sigma}\left(>E_{p}\right)} \cdot \frac{\delta s_{i}}{s_{i}}\right]^{2} \\
& +\sum_{i>i_{p}}\left[1-\frac{\sigma_{i}}{\bar{\sigma}\left(>E_{p}\right)}\right]^{2} \mu_{i}^{2}\left(\frac{\delta \mu_{i}}{\mu_{i}}\right)^{2}+\left(\frac{\delta \sigma_{0}}{\sigma_{0}}\right)^{2}
\end{aligned}
$$

where $\mu_{i}=\psi_{i} \Delta E_{i} / \psi\left(>E_{p}\right)$.

\section{5.b. MEASURED REACTION PROBABILITIES}

The measured reaction probabil ity, $R$, is proportional to some detector response mechanism, $D$ :

$$
R=\frac{1}{N G} \cdot[\varepsilon \cdot \mu(\bar{N}, Y, F, \ldots)] \cdot D,
$$


$D=$ observed integral detector response during or after irradiation (e.g., average count rate from a fission chamber, or counts per second at the end of irradiation from a gamma counting system, or total number of permanently registered nuclear particle tracks divided by $T$, the length of the irradiation).

$G(\lambda, t)=$ activation decay rate factor, $\left(s^{-1}\right)$

$$
=\lambda \int_{-T}^{0} \phi(t) e^{\lambda t} d t / \int_{-T}^{0} \phi(t) d t \quad,
$$

where $\phi(t)$ is the irradiation time history of the neutron fluence rate, $T$ is the length of irradiation, and $\lambda$ is the decay constant. For an uninterrupted irradiation of length $T$ at constant fluence rate, $G=[1-\exp (-\lambda T)] / T$. The dimensionless quantity $C=G / \lambda$ is sometimes used in $\mathrm{place}$ of $\mathrm{G}$. For detectors that register reaction rate directly (e.g., fission chambers), G becomes $1 / \pi$.

$N=$ number of detector atoms.

$\varepsilon=$ detection efficiency factor

$\mu=$ composite of response factors exclusive of $\varepsilon / N G$ required to connect observed counts per sec or its equivalent to reaction probability; e.g., effective fraction of detector atoms including neutron self absorption corrections $(\bar{N})$, fission yield $(Y)$, neutron field perturbations $(F)$, isotopic abundance, branching ratio, extrapolation of pulse height distribution, effects of competing reactions, background subtraction, pulse losses, etc. 
Benchmark referencing tests the combined validity of many of the factors included within the bracket of Eq. $(X-10)$. In dosimetry experiments where absolute reaction probabilities are not determined, or are separately interpreted, a comprehensive calibration factor may be established on the basis of a standard neutron field exposure. In this way, most of the response factors in Eq. $(X-10)$, including the number of detector atoms, are either el iminated or their error contribution reduced.

When the specific purpose of benchmark referencing is to determine a neutron fluence in a neutron field under study, the calibration procedure is referred to as neutron fluence transfer and may be expressed as follows,

$$
\frac{\Phi_{\text {study }}}{\Phi_{\text {bmk }}}=\frac{R_{\text {study }}}{R_{\text {bmk }}} \cdot \frac{\bar{\sigma}_{\text {bmk }}}{\bar{\sigma}_{\text {study }}}=\frac{R_{\text {study }}}{R_{\text {bmk }}} \cdot \frac{\sigma_{\text {bmk }}\left(>E_{p}\right)}{\sigma_{\text {study }}\left(>E_{p}\right)} \cdot \frac{\psi_{b m k}\left(>E_{p}\right)}{\psi_{\text {study }}\left(>E_{p}\right)}
$$

by substituting the observed reaction probability, $R$, from Eq. $(X-10)$ for $R_{C}$ in Eq. $(X-1)$. In many cases of interest the reaction probability ratio is equivalent, or nearly so, to the ratio of the observed integral detector responses, $D_{\text {study }} / D_{\text {bmk }}$, with consequent reduction of uncertainties. The uncertainty in the cross section ratio will depend upon spectrum differences between the benchmark and study neutron fields as described by Eq. $(X-8 a)$.

The important observed quantities for reactor dosimetry are the ratios of reaction probabilities among detectors exposed, or corrected, to an equivalent to total fluence. According to Eq. $(X-1)$, these are simply spectrum-average cross section ratios, and when they are sensitive to spectrum shape, they are 
called spectral indexes as noted in Section 5.a.2. An observed spectral index is obtained from the measured reaction probabilities of two detectors by replacing the calculated reaction probabilities in $\mathrm{Eq} .(\mathrm{X}-1)$ with the measured values from Eq. $(X-10)$ (normalized to the same neutron fluence) and dividing:

$$
\left[S_{\alpha / \beta}\right]_{\text {obs }}=\left(R_{\alpha} / R_{\beta}\right)_{\text {obs }} .
$$

\section{5.c. MEASURED CROSS SECTIONS AND CALCULATED-TO-OBSERVED RATIOS}

For the two fission spectrum benchmarks described in this compendium, the primary integral detector quantities of interest are cross sections and spectral indexes taken with respect to the $238 \mathrm{U}(\mathrm{n}, \mathrm{f})$ reaction. For a natural neutron source such as ${ }^{252} \mathrm{Cf}$, free-field neutron fluences may be established directly and absolute integral cross sections may be determined without recourse to microscopic nuclear data. A substantial amount of such data exists in spite of the relatively low fluence rates available. Data for the ${ }^{252} \mathrm{Cf}$ fission spectrum are reported and summarized in references [Ad77a], $[\mathrm{A} 175 \mathrm{a}],[\mathrm{Al} 75 \mathrm{~b}],[\mathrm{Da} 78 \mathrm{a}],[\mathrm{Fl77a}],[\mathrm{Gi75a}],[\mathrm{Gi77a}],[\mathrm{Gr} 75 \mathrm{a}],[\mathrm{Gr} 82 \mathrm{~b}]$, $[\mathrm{Gr} 83 \mathrm{a}],[\mathrm{He} 75 \mathrm{a}],[\mathrm{He} 76 \mathrm{a}],[\mathrm{Ma} 78 \mathrm{a}],[\mathrm{Ma} 81 \mathrm{a}],[\mathrm{Ma} 82 \mathrm{a}],[\mathrm{Ma} 83 \mathrm{a}],[\mathrm{Ma} 84 \mathrm{a}]$, [Sp77a], [Wa79a], and [Wi81a]. Collections of evaluated measured cross sections may be found in references [Fa75a], [Fa78a], [Ma80a], [Ma81a], [Ma82a] and [Ma83a].

A new and restricted evaluation of cross-section measurements reported by June 1983, was performed at NBS for detector reactions considered important for reactor technology. Results are given in Table $x-9$ and $X-11$. The spectral 
indexes in Table $x-11$ are based on true ratio measurements or when necessary on independent measurements of each reaction. The former is most common for the fission reactions. The uncertainties given in the Tables are one standard deviation and include estimates of systematic errors.

Calculated-to-observed ratios of reaction cross sections are shown in Table $X-10(B 5)$. The errors in calculated cross sections correspond to ${ }^{252} \mathrm{Cf}$ fission spectrum uncertainties from Table $X-5$ propagated according to the second term of Eq. $(X-7)$ in Section 5.a. Two reactions, ${ }^{47} T i(n, p)$ and ${ }^{232} \operatorname{Th}(n, f)$, show calculated-to-observed discrepancies in excess of $10 \%$ and six of the eighteen reactions, some of considerable significance for reactor dosimetry and neutron fluence monitoring, are discrepant by $5 \%$ or more. The standard deviation of the seventeen $C / E$ ratios in Table $X-10(B 5)$, excluding ${ }^{47} \mathrm{Ti}(n, p)$ and ${ }^{232} \mathrm{Th}(n, f)$, are \pm 0.025 about an average departure from unity of 1.034.

Calculated-to-observed ratios of spectral indexes, $\mathrm{C}_{\alpha / \mathrm{U} 8}$, are presented in Table $X-12(B 5)$. The calculated indexes are from Table $X-7$. Contributing errors for observed and calculated indexes are listed separately in columns 4 and 5. As is appropriate for cross section validation, propagated errors for the calculated indexes assess only the contribution of fission spectrum uncertainties given in Table $x-5$. The propagation formula for this partial error is that of the second term of Eq. $(X-8)$, Section 5.a.3. 
The $C_{\alpha / \text { U8 }}$ ratios in Table $X-12(B 5)$ are a further indication of how calibration in a benchmark neutron field can improve the accuracy of a neutron fluence measurement performed with integral detectors. For example, the $C_{\alpha / U 8}$ value for ${ }^{47} \mathrm{Ti}(n, p)$ of 1.29 suggests a serious problem with reaction cross section data, and the $C_{U 8 / \beta}$ value of 0.946 for $235 \mathrm{U}$ fission is a significant disagreement considering the amount of cross section data which exists and the accuracy which is expected of the two fission reactions involved. Without benchmark calibration, a neutron fluence obtained with these detectors would be biased to the extent of the departures from unity. 


\section{LIST OF TABLES}

Table $x-1$

Table $x-2$

Table $x-3$

Table $x-4$

Table $x-5$

Table $x-6(B 5)$

Table $x-7(B 5)$

Table $\mathrm{X}-8(\mathrm{~B} 5)$

Table $x-9$

Table $\mathrm{X}-10$

Table $\mathrm{X}-11$

Table $\mathrm{X}-12(\mathrm{~B} 5)$
${ }^{252}$ Cf Fission Neutron Field Parameters and Error Components

3. a

$3 . \mathrm{a}$ Perturbations in Location B Due to Neutron Scattering (5-cm distance)

Differential Spectrum Measurements for NBS Evaluation of ${ }^{252} \mathrm{Cf}$ and ${ }^{235} \mathrm{U}$ Fission Neutron Spectra

$4 \cdot b \cdot 2$

Evaluated Fission Neutron Spectra for ${ }^{252} \mathrm{Cf}$ and ${ }^{235} \mathrm{U}$ in 45-Group Format

4.b. 3

Error Estimates for ${ }^{252} \mathrm{Cf}$ and $235 \mathrm{U}$ Evaluated Fission Neutron Spectra

$4 \cdot b \cdot 3$

$5 . \mathrm{a}$

$5 \cdot a \cdot 2$

$5 \cdot a \cdot 2$

$5 . c$

$5 \cdot C$

$5 \cdot \mathrm{C}$

5.c 
TABLE $X-1 . \quad{ }^{252} \mathrm{Cf}$ FISSION NEUTRON FIELD PARAMETERS AND ERROR COMPONENTS

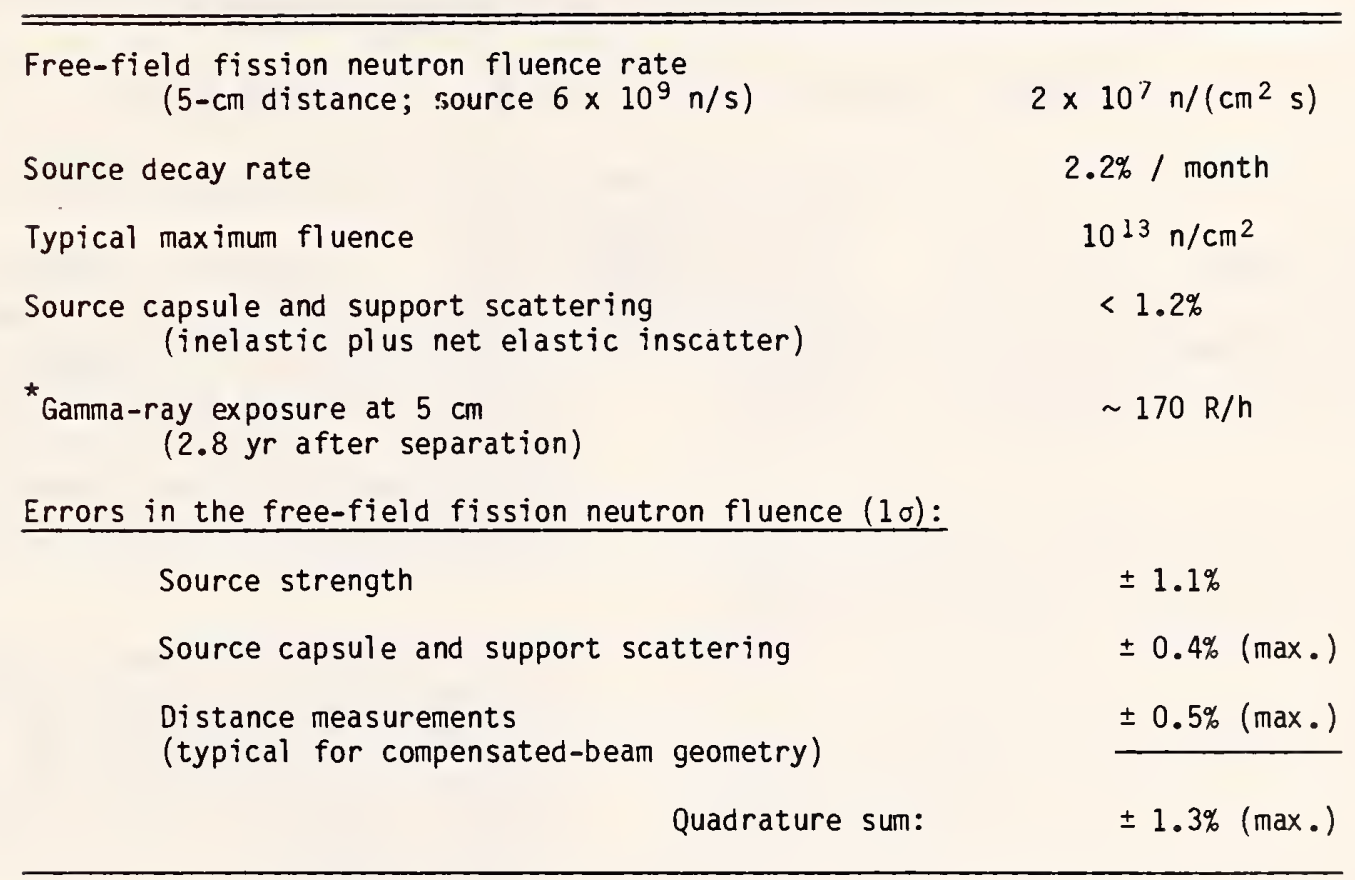

* This estimate does not include $x$-rays which the light-weight encapsulation may not absorb.

TABLE X-2. TYPICAL NEUTRON FLUENCE AND DETECTOR RESPONSE PERTURBATIONS IN LOCATION B DUE TO NEUTRON SCATTERING (5-CM DISTANCE)

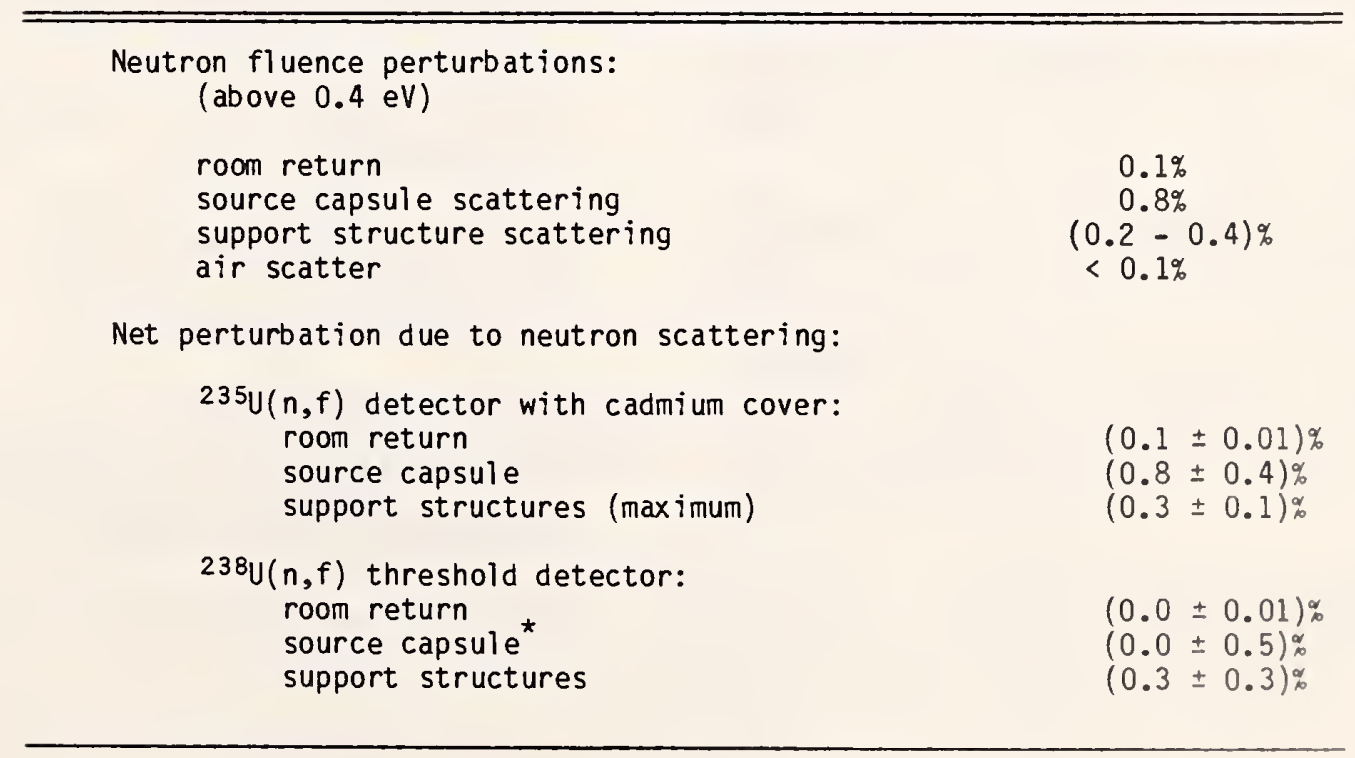

\footnotetext{
* Source capsule perturbations depend markedly upon detector threshold.
} 
TABLE $X-3$. DIFFERENTIAL SPECTRUM MEASUREMENTS FOR NBS EVALUATION OF ${ }^{252} \mathrm{CF}$ AND $235 \mathrm{U}$ FISSION NEUTRON SPECTRA

$$
{ }^{252} \mathrm{CF} \text { S P ONTANEOUS FISSION }
$$

\begin{tabular}{|c|c|c|c|c|}
\hline \multicolumn{2}{|c|}{ Reference } & \multicolumn{2}{|c|}{$\begin{array}{l}\text { Measurement } \\
\text { Range (MeV) }\end{array}$} & Detection Scheme \\
\hline$[\mathrm{Gr} 73 \mathrm{a}]$ & Green et al. & 0.5 & -13 & $\begin{array}{l}\text { Time-of-flight (TOF); energy scale } \\
\text { based on carbon scattering } \\
\text { resonances. Scintillator NE-213 } \\
\text { efficiency from long counter } \\
\text { response (E }<4 \mathrm{MeV}) \text {; calculations } \\
\text { for } \mathrm{E}>4 \mathrm{MeV} \text {. }\end{array}$ \\
\hline [Gr73a] & Knitter et al. & & & $\begin{array}{l}\text { TOF; energy scale calibrated with } \\
\text { monoenergetic neutrons ( } 0.4 \text { to } \\
2.3 \mathrm{MeV}) \text {. Liq. scint. eff. from } \\
\text { angular distribution of } T(p, n) \text {, } \\
D(d, n), L i(p, n), H(n, n) \text {, etc. }(0.05 \\
\text { to } 8.2 \mathrm{MeV}) \text {. }\end{array}$ \\
\hline [We72a] & $\begin{array}{l}\text { Werle and Bluhm } \\
\qquad(\mathrm{n}, \mathrm{p}) \\
(3 \mathrm{He})\end{array}$ & $\begin{array}{l}1.5 \\
1\end{array}$ & $\begin{array}{l}-7 \\
-\quad 4\end{array}$ & $\begin{array}{l}\text { Proton-recoil proportional counters } \\
(P R) \text {; response functions from } \\
\text { monoenergetic response. } \\
\text { 3He-spectrometer and prop. Gas } \\
\text { counter. }\end{array}$ \\
\hline
\end{tabular}

[Gr73a] Meyer et al.

$0.05-8$

PR (0.05 to $1.2 \mathrm{MeV})$. Scint. NE-213; unfolded spectrum (1.1 to $8 \mathrm{MeV})$.

[Gr73a] Jeki et al.

$0.002-1$

TOF; ${ }^{6}$ Li glass scint. efficiency same as Meadows.

[We72a] Meadows

$0.003-15$

TOF; ${ }^{6} \mathrm{Li}$ glass scint. $(\mathrm{E}<2.6 \mathrm{MeV})$;

liq. scint. ( $>1.0 \mathrm{MeV})$;

calculated efficiencies.

[We72a] Conde and During $0.07-7.5$

TOF; ${ }^{6} \mathrm{Li}$ Glass scint. (E $\left.<1.1 \mathrm{MeV}\right)$, Calc. eff.; NE-102A plastic scint. ( $\mathrm{E}>.3 \mathrm{MeV}$ ), eff. Measured against calibrated long counter.

[We72a] Zamyatnin et al. $0.04-6$ TOF; ${ }^{6}$ Li glass and plastic scint. 
TABLE $X-3$. DIFFERENTIAL SPECTRUM MEASUREMENTS FOR NBS EVALUATION OF ${ }^{252} \mathrm{CF}$ AND $235 \mathrm{U}$ FISSION NEUTRON SPECTRA (Continued)

${ }^{235} U$ THERMAL-NEUTRON-INDUCED F I S S I ON

\begin{tabular}{llll}
\hline Reference & $\begin{array}{l}\text { Measurement } \\
\text { Range (MeV) }\end{array}$ & \multicolumn{1}{c}{ Detection Scheme } \\
\hline [Cr56a] Rosen & $0.3-13$ & $\begin{array}{l}\text { Incident neutron energy ( } E_{i n}= \\
\text { thermal. Photographic plate method, } \\
\text { background measurements included. }\end{array}$ \\
[Is73a] Islam and Knitter $0.55-7$ & $\begin{array}{l}E_{\text {in }}=0.4 \mathrm{MeV}, \text { TOF; energy scale } \\
\text { checked with monoenergetic neutrons. } \\
\text { NE-102 scint. eff. from } \mathrm{H}(\mathrm{n}, \mathrm{n}) \text {, up } \\
\text { to } 5.75 \mathrm{MeV}) .\end{array}$
\end{tabular}

[Cr56a] Barnard et al. $\quad 0.1-4 \quad E_{i n}=0.1 \mathrm{MeV}$, TOF; Plastic scint. calibrated with long ctr. $(\mathrm{E}<2.3 \mathrm{MeV})$.

[We72a] Conde and During $1-7.5 E_{\text {in }}=0.04 \mathrm{MeV}$, TOF; Plastic scint. only, same as for ${ }^{252} \mathrm{Cf}$ by Conde and During.

[We72a] Werle and Bluhm

$$
\left(\begin{array}{l}
n, p) \\
(3 \mathrm{He})
\end{array}\right.
$$$$
\begin{aligned}
& 1.5-7 \\
& 1-4
\end{aligned}
$$

Same as for ${ }^{252} \mathrm{Cf}$ by Werle and Bluhm. $E_{\text {in }}=$ thermal.

[Cr56a] Cranberg et a 1 .

$0.18-2.7$ $E_{j n}=0.08 \mathrm{MeV}$, TOF; Plastic scint. calibrated against long ctr. $(0.1-3.4 \mathrm{MeV})$.

[Is73a] Johansson et al.

$0.5-14$

$E_{\text {in }}=0.1-2.0 \mathrm{MeV}$, TOF; energy scale based on scattering resonances $(0.5-21 \mathrm{MeV})$. NE-213 scint. eff. from $H(n, n)$ and $T(p, n)$ angular distribution.

[Cr56a] Watt $2.6-16$ $E_{i n}=$ thermal. Proton-recoil, gas counter telescope. 
TABLE X-4. EVALUATED FISSION NEUTRON SPECTRA FOR ${ }^{252} \mathrm{CF}$ AND ${ }^{235} \mathrm{U}$ IN 45-GROUP FORMAT

\begin{tabular}{|c|c|c|c|c|}
\hline & Designat & $X C F-5-N 1$ & Designat & $X \cup 5-5-N 1$ \\
\hline & & ${ }^{2} \mathrm{Cf}$ & & $235 U$ \\
\hline $\begin{array}{l}\text { Lower } \\
\text { Energy } \\
\text { Boundary } \\
(\mathrm{MeV})\end{array}$ & $\begin{array}{l}\text { Group } \\
\text { Fluence } \\
\phi \cdot \Delta E\end{array}$ & $\begin{array}{l}\text { Cumulative }(a) \\
\text { Fluence to Lower } \\
\text { Boundary }\end{array}$ & $\begin{array}{l}\text { Group } \\
\text { Fluence } \\
\phi \cdot \Delta \mathrm{E}\end{array}$ & $\begin{array}{l}\text { Cumulative } \\
\text { Fluence to Lower } \\
\text { Boundary }\end{array}$ \\
\hline 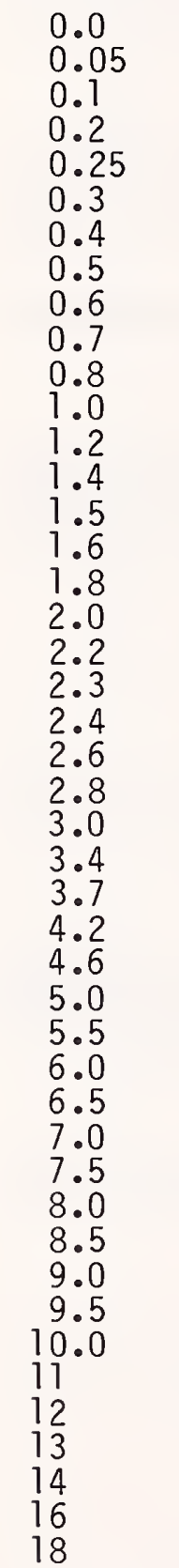 & $\begin{array}{l}0.0039 \\
0.0074 \\
0.0219 \\
0.0140 \\
0.0152 \\
0.0323 \\
0.0337 \\
0.0343 \\
0.0343 \\
0.0338 \\
0.0664 \\
0.0641 \\
0.0608 \\
0.0290 \\
0.0279 \\
0.0527 \\
0.0484 \\
0.0442 \\
0.0206 \\
0.0196 \\
0.0364 \\
0.0328 \\
0.0296 \\
0.0503 \\
0.0310 \\
0.0413 \\
0.0253 \\
0.0200 \\
0.0190 \\
0.0140 \\
0.0102 \\
0.00734 \\
0.00527 \\
0.00378 \\
0.00270 \\
0.00193 \\
0.00137 \\
0.00098 \\
0.00118 \\
0.00059 \\
0.00030 \\
0.00015 \\
0.00011 \\
0.00003 \\
0.00000\end{array}$ & $\begin{array}{l}1.0000 \\
0.9961 \\
0.9887 \\
0.9668 \\
0.9528 \\
0.9377 \\
0.9054 \\
0.8717 \\
0.8374 \\
0.8031 \\
0.7693 \\
0.7029 \\
0.6388 \\
0.5780 \\
0.5490 \\
0.5211 \\
0.4684 \\
0.4200 \\
0.3758 \\
0.3552 \\
0.3356 \\
0.2992 \\
0.2664 \\
0.2368 \\
0.1865 \\
0.1555 \\
0.1142 \\
0.0889 \\
0.0689 \\
0.0499 \\
0.0359 \\
0.0257 \\
0.01839 \\
0.01312 \\
0.00934 \\
0.00664 \\
0.00471 \\
0.00334 \\
0.00236 \\
0.00118 \\
0.00059 \\
0.00029 \\
0.00014 \\
0.00003 \\
0.00000\end{array}$ & $\begin{array}{l}0.0048 \\
0.0088 \\
0.0249 \\
0.0154 \\
0.0166 \\
0.0350 \\
0.0363 \\
0.0367 \\
0.0365 \\
0.0358 \\
0.0697 \\
0.0668 \\
0.0629 \\
0.0298 \\
0.0286 \\
0.0536 \\
0.0487 \\
0.0441 \\
0.0204 \\
0.0193 \\
0.0356 \\
0.0319 \\
0.0284 \\
0.0477 \\
0.0290 \\
0.0378 \\
0.0227 \\
0.0176 \\
0.0164 \\
0.0118 \\
0.00831 \\
0.00573 \\
0.00394 \\
0.00271 \\
0.00186 \\
0.00127 \\
0.00087 \\
0.00059 \\
0.00068 \\
0.00031 \\
0.00014 \\
0.00007 \\
0.00004 \\
0.00001 \\
0.00000\end{array}$ & $\begin{array}{l}1.0000 \\
0.9952 \\
0.9864 \\
0.9615 \\
0.9461 \\
0.9295 \\
0.8945 \\
0.8582 \\
0.8215 \\
0.7851 \\
0.7493 \\
0.6796 \\
0.6128 \\
0.5499 \\
0.5201 \\
0.4915 \\
0.4379 \\
0.3892 \\
0.3451 \\
0.3247 \\
0.3054 \\
0.2698 \\
0.2379 \\
0.2095 \\
0.1618 \\
0.1328 \\
0.09503 \\
0.07233 \\
0.05473 \\
0.03833 \\
0.02653 \\
0.01822 \\
0.01249 \\
0.00855 \\
0.00584 \\
0.00398 \\
0.00271 \\
0.00184 \\
0.00125 \\
0.00057 \\
0.00026 \\
0.00012 \\
0.00005 \\
0.00001 \\
0.00000\end{array}$ \\
\hline
\end{tabular}

For interpolation up to $10 \mathrm{MeV}$, use the shape function $E^{\frac{1}{2}} \cdot \exp (-\mathrm{aE})$; $\mathrm{a}=0.70$ for ${ }^{252} \mathrm{Cf} ; \mathrm{a}=0.76$ for $235 \mathrm{U}$.

(a) Spectrum fraction above lower energy bound. 
TABLE $X-5$. ERROR ESTIMATES FOR $252 \mathrm{Cf}$ and $235 \mathrm{U}$ EVALUATED FISSION NEUTRON SPECTRA

\begin{tabular}{|c|c|c|c|c|c|c|}
\hline \multirow[b]{2}{*}{$\begin{array}{c}\text { Energy } \\
\text { Boundaries }\end{array}$} & \multicolumn{3}{|c|}{$\begin{array}{l}{ }^{252} \mathrm{Cf} \\
\text { (Spontaneous Fission) }\end{array}$} & \multicolumn{3}{|c|}{$\begin{array}{c}235 \mathrm{U} \\
\text { (Thermal-Neutron-Induced Fission) } \\
\text { Error }\end{array}$} \\
\hline & $\begin{array}{l}\text { Group } \\
\text { Fluence } \\
\phi \cdot \Delta E\end{array}$ & $\begin{array}{l}1 \sigma \\
(\%)\end{array}$ & $\begin{array}{l}2 \sigma \\
(\%)\end{array}$ & $\begin{array}{l}\text { Group } \\
\text { Fluence } \\
\phi \cdot \Delta \mathrm{E}\end{array}$ & $\begin{array}{l}1 \sigma \\
(\%)\end{array}$ & $\begin{array}{l}2 \sigma \\
(\%)\end{array}$ \\
\hline 0.0 & & & & & & \\
\hline 0.25 & 0.047 & \pm 13 & \pm 26 & 0.054 & \pm 16 & \pm 32 \\
\hline 08 & 0.184 & \pm 1.1 & \pm 3.3 & 0.197 & \pm 4.1 & \pm 6.2 \\
\hline 1 & 0.220 & \pm 1.8 & \pm 3.6 & 0.229 & \pm 3.0 & \pm 4.8 \\
\hline 1.5 & 0.194 & \pm 1.0 & \pm 3.1 & 0.195 & \pm 3.1 & \pm 5.2 \\
\hline 2.3 & 0.200 & \pm 2.0 & \pm 3.0 & 0.192 & \pm 2.0 & \pm 3.0 \\
\hline 3.7 & 0.146 & \pm 2.1 & \pm 4.8 & 0.127 & \pm 4.8 & \pm 8.0 \\
\hline $\begin{array}{l}12 \\
20\end{array}$ & $\begin{array}{c}0.0087 \\
(0.00058)\end{array}$ & \pm 8.5 & \pm 17 & $\begin{array}{c}0.0056 \\
(0.00026)\end{array}$ & \pm 5.3 & \pm 11 \\
\hline
\end{tabular}


TABLE $X-6(B 5)$. INTEGRAL DETECTOR RESPONSE PARAMETERS

Spectrum: NBS-Evaluated ${ }^{252} \mathrm{Cf}$ Spontaneous Fission

Cross Sections: ENDF/B-V Dosimetry File [EN79a]
Designation: XCF-5-N1

Entry Date: May, 1978

Revised: March, 1982

\begin{tabular}{|c|c|c|c|c|c|c|}
\hline $\begin{array}{l}\text { Detector } \\
\text { Reaction }\end{array}$ & $\begin{array}{r}\text { Cross Se } \\
\frac{\sigma(>E}{E_{p}=0.4 e V ~ E}\end{array}$ & $\begin{array}{l}\text { ction }(a) \\
\frac{p}{p}(p=0.95)\end{array}$ & $\begin{array}{l}\text { Spectrum }(b) \\
\text { Fraction } \\
\psi\left(>E_{95}\right)\end{array}$ & $\begin{array}{l}\text { Median }(c) \\
\text { Response } \\
\text { Energy } \\
E_{p}(p=0.5)\end{array}$ & $\frac{\text { Response }}{E_{p}(p=0.95)}$ & $\frac{\text { Range }(c)}{E_{p}(p=0.05)}$ \\
\hline Spectrum Check & (barns) & (barns) & & $(\mathrm{MeV})$ & $(\mathrm{MeV})$ & $(\mathrm{MeV})$ \\
\hline $\begin{array}{l}\text { Constant } \\
\text { recip. vel., }(l / v)(d)\end{array}$ & $\begin{array}{l}1.000 \\
0.1107\end{array}$ & $\begin{array}{l}1.000 \\
0.1062\end{array}$ & $\begin{array}{l}0.950 \\
0.991\end{array}$ & $\begin{array}{l}1.68 \\
0.995\end{array}$ & $\begin{array}{l}0.260 \\
0.089\end{array}$ & $\begin{array}{l}5.5 \\
4.3\end{array}$ \\
\hline 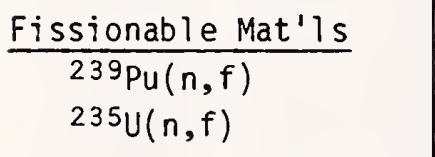 & $\begin{array}{l}1.792 \\
1.236\end{array}$ & $\begin{array}{l}1.807 \\
1.223\end{array}$ & $\begin{array}{l}0.942 \\
0.960\end{array}$ & $\begin{array}{l}1.75 \\
1.68\end{array}$ & $\begin{array}{l}0.286 \\
0.224\end{array}$ & $\begin{array}{l}5.7 \\
5.8\end{array}$ \\
\hline $\begin{array}{l}233 U(n, f) \\
238 U(n, f)\end{array}$ & $\begin{array}{l}1.904 \\
0.3136\end{array}$ & $\begin{array}{l}1.888 \\
0.541\end{array}$ & $\begin{array}{l}0.958 \\
0.550\end{array}$ & $\begin{array}{l}1.62 \\
2.79\end{array}$ & $\begin{array}{l}0.232 \\
1.50\end{array}$ & $\begin{array}{l}5.5 \\
7.2\end{array}$ \\
\hline $\begin{array}{l}23^{7} \mathrm{~Np}(n, f) \\
232 \operatorname{Th}(n, f)\end{array}$ & $\begin{array}{l}1.352 \\
7.81 E-2\end{array}$ & $\begin{array}{l}1.591 \\
0.1360\end{array}$ & $\begin{array}{l}0.807 \\
0.545\end{array}$ & $\begin{array}{l}2.06 \\
3.01\end{array}$ & $\begin{array}{l}0.69 \\
1.51\end{array}$ & $\begin{array}{l}6.2 \\
7.7\end{array}$ \\
\hline $\begin{array}{l}240 \mathrm{Pu}(n, f) \\
24 \mathrm{lPu}(n, f)\end{array}$ & $\begin{array}{l}1.356 \\
1.595\end{array}$ & $\begin{array}{l}1.598 \\
1.575\end{array}$ & $\begin{array}{l}0.806 \\
0.962\end{array}$ & $\begin{array}{l}2.06 \\
1.63\end{array}$ & $\begin{array}{l}0.69 \\
0.22\end{array}$ & $\begin{array}{l}6.1 \\
5.6\end{array}$ \\
\hline $\begin{array}{l}238 U(n, \gamma) \\
232 \operatorname{Th}(n, \gamma)\end{array}$ & $\begin{array}{l}6.83 E-2 \\
8.97 E-2\end{array}$ & $\begin{array}{l}6.55 \quad E-2 \\
8.63 E-2\end{array}$ & $\begin{array}{l}0.991 \\
0.987\end{array}$ & $\begin{array}{l}0.90 \\
0.92\end{array}$ & $\begin{array}{l}0.088 \\
0.109\end{array}$ & $\begin{array}{l}2.8 \\
2.9\end{array}$ \\
\hline $\begin{array}{c}\text { Capture Reactions } \\
{ }^{23} \mathrm{Na} \\
{ }^{45} \mathrm{SC} \\
58 \mathrm{Fe}\end{array}$ & $\begin{array}{l}0.2712 \quad E-3 \\
5.26 \quad E-3 \\
1.660 \quad E-3\end{array}$ & $\begin{array}{ll}0.259 & E-3 \\
5.01 & E-3 \\
1.587 & E-3\end{array}$ & $\begin{array}{l}0.996 \\
0.997 \\
0.994\end{array}$ & $\begin{array}{l}0.95 \\
0.65 \\
1.07\end{array}$ & $\begin{array}{l}0.055 \\
0.041 \\
0.067\end{array}$ & $\begin{array}{l}4.7 \\
2.9 \\
4.8\end{array}$ \\
\hline $\begin{array}{l}{ }^{59} \mathrm{Co} \\
{ }^{63} \mathrm{Cu}\end{array}$ & $\begin{array}{l}6.03 E-3 \\
9.65 E-3\end{array}$ & $\begin{array}{l}5.76 \quad E-3 \\
9.22 \quad E-3\end{array}$ & $\begin{array}{l}0.994 \\
0.994\end{array}$ & $\begin{array}{l}1.08 \\
0.98\end{array}$ & $\begin{array}{l}0.068 \\
0.067\end{array}$ & $\begin{array}{l}4.0 \\
4.0\end{array}$ \\
\hline $\begin{array}{l}115 \mathrm{In} \\
{ }^{197} \mathrm{Au}\end{array}$ & $\begin{array}{l}0.1212 \\
0.0763\end{array}$ & $\begin{array}{l}0.1169 \\
0.0730\end{array}$ & $\begin{array}{l}0.986 \\
0.994\end{array}$ & $\begin{array}{l}1.12 \\
0.75\end{array}$ & $\begin{array}{l}0.117 \\
0.068\end{array}$ & $\begin{array}{l}3.0 \\
3.0\end{array}$ \\
\hline
\end{tabular}


TABLE $X-6(B 5)$. INTEGRAL DETECTOR RESPONSE PARAMETERS (Continued)

Spectrum: NBS-Evaluated ${ }^{252}$ Cf Spontaneous Fission

Designation: $\quad X C F-5-N 1$

Entry Date: May, 1978

Cross Sections: ENDF/B-V Dosimetry File [EN79a]

Revised: March, 1982

\begin{tabular}{|c|c|c|c|c|c|c|}
\hline $\begin{array}{l}\text { Detector } \\
\text { Reaction }\end{array}$ & $\begin{array}{r}\text { Cross Sec } \\
\text { ol }>\mathrm{E}_{p} \\
\end{array}$ & $\operatorname{tion}(a)$ & $\begin{array}{l}\text { Spectrum } \\
\text { Fraction }\end{array}$ & $\begin{array}{l}\text { Median }(c) \\
\text { Response } \\
\text { Energy }\end{array}$ & Response & Range $^{(c)}$ \\
\hline & $E_{p}=0.4 e V$ & $E_{p}(p=0.95)$ & $\psi\left(>E_{95}\right)$ & $E_{p}(p=0.5)$ & $E_{p}(p=0.95)$ & $E_{p}(p=0.05)$ \\
\hline Helium Production & (barns) & (barns) & & $(\mathrm{MeV})$ & $(\mathrm{MeV})$ & $(\mathrm{MeV})$ \\
\hline${ }^{10} B(n, \alpha)$ & 0.489 & 0.468 & 0.992 & 1.44 & 0.078 & 5.6 \\
\hline${ }^{6} \operatorname{Li}(n, \alpha)$ & 0.465 & 0.454 & 0.972 & 1.55 & 0.179 & 6.0 \\
\hline Threshold Reactions & & & & & & \\
\hline $115 \operatorname{In}\left(n, n^{\prime}\right)$ & 0.1819 & 0.270 & 0.641 & 2.71 & 1.20 & 6.2 \\
\hline${ }^{47} \mathrm{Ti}(n, p)$ & $0.2407 \quad E-1$ & 0.520 E-1 & 0.440 & 3.9 & 1.92 & 8.0 \\
\hline${ }^{32} S(n, p)$ & 0.0760 & 0.206 & 0.350 & 4.1 & 2.3 & 7.9 \\
\hline${ }^{58} \mathrm{Ni}(\mathrm{n}, \mathrm{p})$ & 0.1138 & 0.285 & 0.380 & 4.2 & 2.2 & 7.9 \\
\hline${ }^{54} \mathrm{Fe}(\mathrm{n}, \mathrm{p})$ & $8.83 E-2$ & 0.249 & 0.337 & 4.3 & 2.4 & 8.0 \\
\hline${ }^{46} T i(n, p)$ & $1.347 \mathrm{E}-2$ & $8.85 \mathrm{E}-2$ & 0.1445 & 5.9 & 3.8 & 9.9 \\
\hline${ }^{27} A \ell(n, p)$ & 5.14 E-3 & $2.79 E-2$ & 0.1749 & 6.0 & 3.5 & 9.8 \\
\hline${ }^{56} \mathrm{Fe}(n, p)$ & 1.414 E-3 & $2.79 E-2$ & 0.0482 & 7.6 & 5.6 & 12 \\
\hline${ }^{63} \mathrm{Cu}(n, \alpha)$ & $0.758 E-3$ & $9.72 \mathrm{E}-3$ & 0.0741 & 7.7 & 4.9 & 12 \\
\hline${ }^{27} A l(n, \alpha)$ & $1.059 E-3$ & $4.08 E-2$ & 0.0247 & 8.6 & 6.6 & 12 \\
\hline${ }^{48} \mathrm{Ti}(n, p)$ & $0.409 \mathrm{E}-3$ & $1.099 \mathrm{E}-2$ & 0.0354 & 8.4 & 6.0 & 13 \\
\hline Additions $(\mathrm{e})$ & & & & & & \\
\hline${ }^{103} R h\left(n, n^{\prime}\right)$ & 0.703 & 0.849 & 0.787 & 2.37 & 0.75 & 6.0 \\
\hline${ }^{93} \mathrm{Nb}\left(n, n^{\prime}\right)$ & 0.1605 & 0.220 & 0.694 & 2.61 & 1.02 & 5.9 \\
\hline $60 \mathrm{Ni}(n, p)$ & $3.44 E-3$ & $4.57 E-2$ & 0.0715 & 7.3 & 4.9 & 11 \\
\hline $55 \mathrm{Mn}(n, 2 n)$ & 0.440 E-3 & 0.401 & $1.044 E-3$ & 13 & 11 & 16 \\
\hline $\mathrm{dpa}$ & 895 & 1030 & 0.825 & 2.65 & 0.64 & 6.8 \\
\hline
\end{tabular}


Footnotes for TABLE X-6(B5)

(a) The value given in column 2 is the full-spectrum-averaged cross section above a cadmium cut-off of $0.4 \mathrm{eV}$. The truncated cross section on column 3 is for a truncation energy (column 6) above which $95 \%$ of the detector response occurs. A spectrum-averaged cross section truncated at energy $E_{p}$ is given by

$$
\sigma\left(>E_{p}\right)=\int_{E_{p}}^{\infty} \sigma(E) \psi(E) d E / \int_{E_{p}}^{\infty} \psi(E) d E
$$

(b) The fraction of the spectrum above $E_{95} \equiv E_{p}(p=0.95): \psi\left(>E_{95}\right)=$ $\int_{E_{95}}^{\infty} \psi(E) d E$. The full-spectrum-averaged cross section $\sigma(>0.4 \mathrm{eV})$ is equal to $\sigma\left(>E_{95}\right) \cdot \psi\left(>E_{95}\right) / 0.95$.

(c) The fractions $p=0.95,0.5$, and 0.05 define energies above which $95 \%, 50 \%$ (median), and $5 \%$ of the detector response occurs, respectively. $E_{p}$ is defined by the relation

$$
\int_{E_{p}}^{\infty} \sigma(E) \psi(E) d E=p \cdot[\sigma(>0.4 \text { eV })]
$$

where $E_{p}(p=1)=0.4 \mathrm{eV}$, and $E(p=0)=20 \mathrm{MeV} ;$ and $\int_{0.4}^{\infty} \mathrm{eV} \psi(E) \mathrm{dE}=1$.

(d) Normal ization is $\int_{0.4 \mathrm{eV}}^{18 \mathrm{MeV}} \sigma_{1 / \mathrm{V}} \mathrm{E} \psi(\mathrm{E}) \mathrm{dE}=1$.

(e) Cross sections not taken from the ENDF/BV Dosimetry File:

- $N b\left(n, n^{\prime}\right)$ is from the IRDF Dosimetry File [Cu80a].

- $R h\left(n, n^{\prime}\right)$ is from data reported in Ref. [Pa80a].

- dpa, the atom displacement cross section, is from ASTM Standard Practice E693-79 [An83a]. 
TABLE $X-7(B 5)$. CALCULATED SPECTRAL INDEXES

Spectrum: ${ }^{252} \mathrm{Cf}$ Spontaneous Fission (NBS evaluation, $[\mathrm{Gr} 75 \mathrm{~b}],[\mathrm{Gr} 75 \mathrm{c}]$ )

Spectral Index: Fu17-Spectrum Cross Section from Table $X-6$ (B5)

Designation: $\quad X C F-5-N 1$ Entry Date: Feb. 1980 (ENDF/B-V Dosimetry File)

Revised: March, 1982

$$
\left[S_{\alpha / \beta}\right]_{c a\} c .} \equiv\left[\bar{\sigma}_{\alpha} / \bar{\sigma}_{\beta}\right]_{\text {calc. }}
$$

\begin{tabular}{|c|c|c|c|c|c|}
\hline$\searrow_{1}^{\beta}$ & $\begin{array}{l}235 U(n, f) \\
=1.236 b)\end{array}$ & $\begin{array}{c}238 U(n, f) \\
(\bar{\sigma}=0.3136 b)\end{array}$ & & $\begin{array}{l}235 U(n, f) \\
(\bar{\sigma}=1.236 b)\end{array}$ & $\begin{array}{c}238 U(n, f) \\
(\bar{\sigma}=0.3136 b)\end{array}$ \\
\hline \multicolumn{3}{|l|}{ Fission } & \multicolumn{3}{|l|}{ Threshold } \\
\hline${ }^{239} \mathrm{Pu}$ & 1.450 & 5.71 & $115 \operatorname{In}\left(n, n^{\prime}\right)$ & 0.1472 & 0.580 \\
\hline $235 U$ & 1.000 & 3.941 & ${ }^{47} T i(n, p)$ & 0.01947 & 0.0768 \\
\hline $233 \mathrm{U}$ & 1.540 & 6.07 & ${ }^{58} \mathrm{Ni}(n, p)$ & 0.0921 & 0.363 \\
\hline $238 \mathrm{U}$ & 0.2537 & 1.000 & ${ }^{54} \mathrm{Fe}(\mathrm{n}, \mathrm{p})$ & 0.0714 & 0.2816 \\
\hline $237 \mathrm{~Np}$ & 1.094 & 4.31 & ${ }^{46} \mathrm{Ti}(n, p)$ & 0.01089 & 0.0430 \\
\hline $240 \mathrm{pu}$ & 1.097 & 4.32 & ${ }^{63} \mathrm{Cu}(n, \alpha)$ & $6.13 E-4$ & 2.417 E-3 \\
\hline \multicolumn{3}{|l|}{ He Prod. } & ${ }^{56} \mathrm{Fe}(n, p)$ & $1.144 \mathrm{E}-3$ & $4.509 E-3$ \\
\hline${ }^{10} B(n, \alpha)$ & 0.395 & 1.559 & ${ }^{48} \mathrm{Ti}(n, p)$ & $3.31 \mathrm{E}-4$ & $1.304 \mathrm{E}-3$ \\
\hline${ }^{6} \operatorname{Li}(n, \alpha)$ & 0.376 & 1.483 & ${ }^{27} \mathrm{~A} \ell(n, \alpha)$ & $8.57 \mathrm{E}-4$ & $3.38 E-3$ \\
\hline \multicolumn{3}{|l|}{ Capture } & \multicolumn{3}{|l|}{ Additions } \\
\hline $238 U$ & 0.0553 & 0.2178 & $103 \mathrm{Rh}\left(n, n^{\prime}\right)$ & 0.569 & 2.242 \\
\hline${ }^{232} \mathrm{Th}$ & 0.0725 & 0.2860 & ${ }^{93} \mathrm{Nb}\left(n, n^{\prime}\right)$ & 0.1299 & 0.511 \\
\hline${ }^{197} \mathrm{Au}$ & 0.0619 & 0.2433 & & & \\
\hline${ }^{59} \mathrm{Co}$ & $4.88 E-3$ & 0.01923 & & & \\
\hline${ }^{58} \mathrm{Fe}$ & $1.343 \mathrm{E}-3$ & $5.29 E-3$ & & & \\
\hline
\end{tabular}


TABLE X-8(B5). CALCULATED SPECTRAL INDEXES - TRUNCATED

Spectrum: ${ }^{252} \mathrm{Cf}$ Spontaneous Fission

Spectral Index: Truncated Cross Section from Table $X-6$ (B5)

(ENDF/B-V Dosimetry File)
Designation: $\quad X C F-5-N 1$

Entry Date: Feb. 1980

Revised: March, 1982

$$
\left[S_{\alpha / \beta}\right]_{c a l c .} \equiv\left[\bar{\sigma}_{\alpha}\left(>E_{95}\right) / \bar{\sigma}_{\beta}\left(>E_{95}\right)\right]_{c a l c .}
$$

\begin{tabular}{|c|c|c|c|}
\hline$B$ & $\begin{array}{c}238 U(n, f) \\
\bar{\sigma}\left(>E_{95}\right)=0.541 b\end{array}$ & & $\begin{array}{c}238 U(n, f) \\
\bar{\sigma}\left(>E_{95}\right)=0.541 b\end{array}$ \\
\hline Fission & & Threshol d & \\
\hline${ }^{239} \mathrm{Pu}$ & 3.34 & $115 \operatorname{In}\left(n, n^{\prime}\right)$ & 0.500 \\
\hline $235 \mathrm{U}$ & 2.261 & ${ }^{47} T i(n, p)$ & 0.0961 \\
\hline $238 \mathrm{U}$ & 1.000 & ${ }^{32} S(n, p)$ & 0.381 \\
\hline $237 \mathrm{~Np}$ & 2.941 & ${ }^{58} \mathrm{Ni}(n, p)$ & 0.527 \\
\hline \multirow[t]{2}{*}{$240 \mathrm{Pu}$} & 2.954 & ${ }^{54} \mathrm{Fe}(n, p)$ & 0.460 \\
\hline & & ${ }^{46} T i(n, p)$ & 0.1636 \\
\hline Additions & & ${ }^{56} \mathrm{Fe}(n, p)$ & 0.0516 \\
\hline${ }^{103} \operatorname{Rh}\left(n, n^{\prime}\right)$ & 1.569 & ${ }^{63} \mathrm{Cu}(n, \alpha)$ & 0.01797 \\
\hline \multirow[t]{2}{*}{${ }^{93} \mathrm{Nb}\left(n, n^{\prime}\right)$} & 0.407 & ${ }^{48} T i(n, p)$ & 0.0203 \\
\hline & & ${ }^{27} \mathrm{Al}(n, \alpha)$ & 0.0754 \\
\hline
\end{tabular}


TABLE X-9. OBSERVED INTEGRAL CROSS SECTIONS

Spectrum: ${ }^{252} \mathrm{Cf}$ Spontaneous Fission

Designation: XCF

Entry Date: May 1978

Revised: June, 1983

\begin{tabular}{|c|c|c|c|}
\hline Reaction & $\begin{array}{l}\text { Cross Section }(\mathrm{a}) \\
\text { Value } \\
\left(\times 10^{-27} \mathrm{~cm}^{2}\right)\end{array}$ & $\begin{array}{l}\text { Median } \\
\text { Response } \\
\text { Energy, } \\
\mathrm{E}_{50}(\mathrm{MeV})\end{array}$ & $\begin{array}{l}\text { Measurement } \\
\text { References }\end{array}$ \\
\hline \multicolumn{4}{|c|}{ Threshol d Reactions } \\
\hline $238 U(n, f)$ & 326. $\pm 2.0 \%$ & 2.8 & {$[G r 83 a],[G i 75 a]$} \\
\hline${ }^{237} \mathrm{~Np}(n, f)$ & $1365 \pm 2.0 \%$ & 2.1 & {$[\operatorname{Gr} 83 a],[G i 75 a]$} \\
\hline $240 \mathrm{Pu}(n, f)$ & $1337 \pm 2.4 \%$ & 2.1 & {$[\mathrm{Gr} 83 \mathrm{a}]$} \\
\hline${ }^{232} \operatorname{Th}(n, f)$ & $89.4 \pm 3.0 \%$ & 3.0 & [Gr83a] \\
\hline${ }^{93} \mathrm{Nb}\left(n, n^{\prime}\right)$ & $149 \pm 7 \%$ & 2.5 & {$[A 182 a]$} \\
\hline${ }^{1} 15 \operatorname{In}\left(n, n^{\prime}\right)$ & 195. $\pm 1.9 \%$ & 2.7 & {$[$ La82a $],[$ Ma79a $]$} \\
\hline${ }^{47} \mathrm{Ti}(n, p)$ & $19.4 \pm 2.5 \%$ & 3.9 & {$[A 175 b],[S p 77 a]$} \\
\hline${ }^{58} \mathrm{Ni}(n, p)$ & $119.4 \pm 1.5 \%$ & 4.2 & {$[$ La82a $],[A 175 b],[F 77 \mathrm{a}]$} \\
\hline${ }^{54} \mathrm{Fe}(n, p)$ & $87.8 \pm 2.2 \%$ & 4.3 & {$[F 777 a],[A 175 b],[S p 77 a]$} \\
\hline${ }^{46} \mathrm{Ti}(n, p)$ & $14.2 \pm 2.5 \%$ & 5.9 & {$[\mathrm{~A} 175 \mathrm{~b}],[\mathrm{Sp} 77 \mathrm{a}]$} \\
\hline${ }^{63} \mathrm{Cu}(n, \alpha)$ & $0.696 \pm 3.0 \%$ & 7.7 & {$[$ Wi81a], [Ma82a $]$} \\
\hline${ }^{56} \mathrm{Fe}(\mathrm{n}, \mathrm{p})$ & $1.45 \pm 2.5 \%$ & 7.6 & {$[A 175 b]$} \\
\hline${ }^{48} \mathrm{Ti}(n, p)$ & $0.425 \pm 2.5 \%$ & 8.4 & {$[\mathrm{~A} 175 \mathrm{~b}],[\mathrm{Sp} 77 \mathrm{a}]$} \\
\hline${ }^{27} \mathrm{Al}(n, \alpha)$ & $1.024 \pm 2.5 \%$ & 8.6 & {$[\mathrm{~A} 175 \mathrm{~b}], \quad[\operatorname{Sp} 77 \mathrm{a}]$} \\
\hline \multicolumn{4}{|c|}{ Non-Threshold Reactions } \\
\hline $19 \overline{\mathrm{Au}(n, \gamma)}$ & $77.4 \pm 2.5 \%$ & 0.74 & {$[\operatorname{Ma} 79 a],[G r 75 a]$} \\
\hline $239 \mathrm{Pu}(n, f)$ & 1824. $\pm 1.9 \%$ & 1.75 & {$[G r 83 a],[G i 75 a]$} \\
\hline $235 U(n, f)$ & $1216 . \pm 1.6 \%$ & 1.68 & $\begin{array}{l}{[\mathrm{Gr} 83 \mathrm{a}],[\mathrm{He} 76 \mathrm{a}],[\mathrm{Gr} 82 \mathrm{~b}],} \\
{[\mathrm{Sp} 80 \mathrm{a}],[\text { Wa79a }],[\mathrm{Da} 78 \mathrm{a}]}\end{array}$ \\
\hline $233 U(n, f)$ & $\pm 2.5 \%$ & 1.62 & {$[\mathrm{Gr} 83 a]$} \\
\hline $241 \mathrm{Pu}(n, f)$ & $\pm 5.0 \%$ & 1.63 & {$[\mathrm{Gr} 83 \mathrm{a}]$} \\
\hline
\end{tabular}

(a) The total error includes a consideration of the agreement among independent measurements when they are available, as well as the error estimate given in the primary reference.

(b) Reference for the primary measurement in the evaluation is listed first. 
TABLE $X-10(B 5)$. CALCULATED-T0-OBSERVED RATIOS OF CROSS SECTIONS

Spectrum: ${ }^{252} \mathrm{Cf}$ Spontaneous Fission (NBS Evaluation)
Designation: $\quad X C F-5-N 1$

Entry Date: March 1982

Revised: June, 1983

Cross Section: Calculated: ENDF/B-V

\begin{tabular}{|c|c|c|c|c|c|}
\hline Reaction & $\begin{array}{r}\text { Cr } \\
\text { Observ } \\
\text { (Table }\end{array}$ & $\begin{array}{l}\text { ross Sec } \\
\text { ved } \\
x-9)\end{array}$ & $\begin{array}{l}\left(10^{-2)} \mathrm{cm}\right. \\
\text { Calcula } \\
\text { (Table }\end{array}$ & $\begin{array}{l}\text { 2) } \\
\text { ted (a) } \\
x-6(B 5)\end{array}$ & $\begin{array}{l}\text { Calculated_(b) } \\
\text { to-Observed }\end{array}$ \\
\hline \multicolumn{6}{|l|}{ Fission } \\
\hline$\overline{239 \mathrm{Pu}}(\mathrm{n}, \mathrm{f})$ & 1824 & $\pm 1.9 \%$ & 1792 & $\pm 0.1 \%$ & $0.982 \pm 1.9 \%$ \\
\hline $235 U(n, f)$ & 1216 & $\pm 1.6 \%$ & 1236 & $\pm 0.1 \%$ & $1.016 \pm 1.6 \%$ \\
\hline $233 U(n, f)$ & 1893 & $\pm 2.5 \%$ & 1904 & $\pm 0.1 \%$ & $1.006 \pm 2.5 \%$ \\
\hline $238 U(n, f)$ & 326 & $\pm 2.0 \%$ & 313.6 & $\pm 0.8 \%$ & $0.962 \pm 2.2 \%$ \\
\hline $237 \mathrm{~Np}(n, f)$ & 1365 & $\pm 2.0 \%$ & 1352 & $\pm 0.6 \%$ & $0.990 \pm 2.1 \%$ \\
\hline $232 \operatorname{Th}(n, f)$ & 89.4 & $\pm 3.0 \%$ & 78.1 & $\pm 0.9 \%$ & $0.874 \pm 3.1 \%$ \\
\hline $240 \mathrm{Pu}(\mathrm{n}, \mathrm{f})$ & 1337 & $\pm 2.4 \%$ & 1356 & $\pm 0.6 \%$ & $1.014 \pm 2.5 \%$ \\
\hline $241 \mathrm{Pu}(\mathrm{n}, \mathrm{f})$ & 1616 & $\pm 5.0 \%$ & 1595 & $\pm 0.2 \%$ & $0.987 \pm 5.0 \%$ \\
\hline \multicolumn{6}{|l|}{ Capture } \\
\hline${ }^{197} \mathrm{Au}(\mathrm{n}, \gamma)$ & 77.4 & $\pm 2.5 \%$ & 76.3 & $\pm 2.0 \%$ & $0.986 \pm 3.2 \%$ \\
\hline \multicolumn{6}{|l|}{ Threshold } \\
\hline${ }^{93} \mathrm{Nb}\left(n, n^{\prime}\right)$ & 149 & $\pm 7 \%$ & 160.5 & $\pm 0.8 \%$ & $1.08 \pm 7 \%$ \\
\hline${ }^{115} \operatorname{In}\left(n, n^{\prime}\right)$ & 195 & $\pm 1.9 \%$ & 182 & $\pm 0.8 \%$ & $0.93 \pm 2.1 \%$ \\
\hline${ }^{47} \mathrm{Ti}(\mathrm{n}, \mathrm{p})$ & 19.4 & $\pm 2.5 \%$ & 24.1 & $\pm 1.1 \%$ & $1.242 \pm 2.7 \%$ \\
\hline${ }^{58} \mathrm{Ni}(n, p)$ & 119.4 & $\pm 1.5 \%$ & 114 & $\pm 1.2 \%$ & $0.955 \pm 1.9 \%$ \\
\hline${ }^{54} \mathrm{Fe}(n, p)$ & 87.8 & $\pm 2.2 \%$ & 88.3 & $\pm 1.4 \%$ & $1.006 \pm 2.6 \%$ \\
\hline${ }^{46} T i(n, p)$ & 14.2 & $\pm 2.5 \%$ & 13.5 & $\pm 2.1 \%$ & $0.95 \pm 3.3 \%$ \\
\hline${ }^{63} \mathrm{Cu}(n, \alpha)$ & 0.696 & $\pm 3.0 \%$ & 0.758 & $\pm 3.7 \%$ & $1.09 \pm 4.8 \%$ \\
\hline${ }^{56} \mathrm{Fe}(n, p)$ & 1.45 & $\pm 2.5 \%$ & 1.414 & $\pm 3.2 \%$ & $0.975 \pm 4.1 \%$ \\
\hline${ }^{27} \mathrm{Al}(n, \alpha)$ & 1.024 & $\pm 2.5 \%$ & 1.050 & $\pm 5.3 \%$ & $1.034 \pm 5.9 \%$ \\
\hline${ }^{48} \mathrm{Ti}(n, p)$ & 0.425 & $\pm 2.5 \%$ & $0.40 \mathrm{~s}$ & $\pm 4.7 \%$ & $0.962 \pm 5.3 \%$ \\
\hline
\end{tabular}

(a) Errors correspond to ${ }^{252} \mathrm{Cf}$ fission spectrum uncertainties (10) given in Table $X-5$ and propagated according to the second term of Eq. X-7, Section $5 . a .3$.

(b) Errors are quadrature sum of errors in columns 2 and 3. 
TABLE $X-11$. OBSERVED SPECTRAL INDEXES

Spectrum: ${ }^{252} \mathrm{Cf}$ Spontaneous Fission

$\left[\mathrm{S}_{\alpha / \mathrm{U} 8}\right]_{\mathrm{obs}}=\left[\bar{\sigma}_{\alpha} / \bar{\sigma}_{\mathrm{f}}(\mathrm{U} 238)\right]_{\mathrm{obs}}$
Designation: XCF

Entry Date: May 1978

Revised: June, 1983

$\begin{array}{llc} & \text { Reaction (b) } \\ \text { Ron-overl ap } & \text { Reactions }(a) & \text { Spectral } \\ \text { Interval (MeV) } & \text { Index }\end{array}$

Threshold Reactions

\begin{tabular}{|c|c|c|c|c|}
\hline $23^{7} \mathrm{~Np}(\mathrm{n}, \mathrm{f}) / \mathrm{U} 8$ & 0.7 & -1.5 & 4.19 & $\pm 1.5 \%$ \\
\hline $240 \mathrm{Pu}(\mathrm{n}, \mathrm{f}) / \mathrm{U} 8$ & 0.7 & -1.5 & 4.10 & $\pm 1.6 \%$ \\
\hline${ }^{115} \operatorname{In}\left(n, n^{\prime}\right) / U 8$ & 1.2 & -1.5 & 0.598 & $\pm 2.8 \%$ \\
\hline${ }^{47} \mathrm{Ti}(\mathrm{n}, \mathrm{p}) / \mathrm{U} 8$ & 1.9 & -1.5 & 0.0595 & $\pm 3.2 \%$ \\
\hline${ }^{58} \mathrm{Ni}(\mathrm{n}, \mathrm{p}) / \mathrm{U} 8$ & 2.2 & -1.5 & 0.366 & $\pm 2.1 \%$ \\
\hline${ }^{54} \mathrm{Fe}(\mathrm{n}, \mathrm{p}) / \mathrm{U} 8$ & 2.4 & -1.5 & 0.269 & $\pm 3.0 \%$ \\
\hline${ }^{46} \mathrm{Ti}(\mathrm{n}, \mathrm{p}) / \mathrm{U} 8$ & 3.8 & -1.5 & 0.0436 & $\pm 3.2 \%$ \\
\hline${ }^{63} \mathrm{Cu}(\mathrm{n}, \alpha) / \mathrm{U} 8$ & 4.9 & -1.5 & $2.14 E-3$ & $\pm 3.6 \%$ \\
\hline${ }^{56} \mathrm{Fe}(\mathrm{n}, \mathrm{p}) / \mathrm{U} 8$ & 5.6 & -1.5 & $4.45 E-3$ & $\pm 3.2 \%$ \\
\hline${ }^{48} \mathrm{Ti}(\mathrm{n}, \mathrm{p}) / \mathrm{U} 8$ & 6.0 & -1.5 & $1.304 \mathrm{E}-3$ & $\pm 3.2 \%$ \\
\hline${ }^{27} \mathrm{Al}(n, \alpha) / U 8$ & 6.6 & -1.5 & $3.14 E-3$ & $\pm 3.2 \%$ \\
\hline
\end{tabular}

Non-Threshol d Reactions

\begin{tabular}{|c|c|c|c|}
\hline $239 \mathrm{Pu}(\mathrm{n}, \mathrm{f}) / \mathrm{U} 8$ & $0.3-1.5$ & 5.60 & $\pm 1.2 \%$ \\
\hline $235 U(n, f) / U 8$ & $0.22-1.5$ & 3.73 & $\pm 1.2 \%$ \\
\hline $233 U(n, f) / U 8$ & $0.23-1.5$ & 5.81 & $\pm 2.1 \%$ \\
\hline $2{ }^{2} \mathrm{Pu}(\mathrm{n}, \mathrm{f}) / \mathrm{U} 8$ & $0.22-1.5$ & 4.96 & $\pm 5.7 \%$ \\
\hline $197 \mathrm{Au}(n, \gamma) / \mathrm{U} 8$ & $0.06-1.5$ & 0.237 & $\pm 3.2 \%$ \\
\hline
\end{tabular}

(a) Al 1 spectral indexes are taken rel ative to the $238 U(n, f)$ reaction 1 abeled "U8".

(b) Non-overl apping energy response interval between the $95 \%$ response energy for each detector (see Table X-6 and footnote (b)).

(c) Fission detector indexes are from simul taneous measurement of fission rates from both isotopes and hence show smaller uncertainties because of reduced neutron fluence errors. Other spectral indexes are formed from independent cross section measurements 1 isted in Table X-9. 
TABLE $X-12(B 5)$. CALCULATED-T0-OBSERVED RATIOS OF SPECTRAL INDEXES - NBS EVALUATION

Spectrum: ${ }^{252} \mathrm{Cf}$ Spontaneous Fission (NBS evaluation, [Gr75b], [Gr75c])

Spectral Index: Calculated: Table $x-7$ (B5)

Observed: Table $X-11$
Designation: $\quad X C F-5-N 1$

Entry Date: March, 1982

Revised: June, 1983

$C_{\alpha / U 8}=\left[s_{\alpha / U 8}\right]_{c a l c .} /\left[s_{\alpha / U 8}\right]_{0 b s}$. or $C_{U 8 / \alpha}$

\begin{tabular}{|c|c|c|c|c|c|}
\hline Reaction (a) & $\begin{array}{l}\text { Reaction (b) } \\
\text { Non-overlap } \\
\text { Interval (MeV) }\end{array}$ & $\begin{array}{l}\text { Calcul ated- } \\
\text { To-0b served } \\
\text { Values }\end{array}$ & $\begin{array}{l}\text { Observed } \\
\text { Value }\end{array}$ & $\begin{array}{l}\text { E R R O R S } \\
\text { Cal cul ated (c) } \\
\text { Value }\end{array}$ & $\begin{array}{l}\text { o) } \\
\text { Total }\end{array}$ \\
\hline \multicolumn{6}{|c|}{ Threshold Reactions } \\
\hline$U 8 / 237 \mathrm{~Np}(\mathrm{n}, f)$ & $0.7-1.5$ & 0.972 & $\pm 1.5 \%$ & $\pm 0.5 \%$ & $\pm 1.7 \%$ \\
\hline $\mathrm{U} 8 / 240 \mathrm{Pu}$ & $0.7-1.5$ & 0.949 & $\pm 1.6 \%$ & $\pm 0.5 \%$ & $\pm 1.7 \%$ \\
\hline${ }^{47} T i(n, p) / 48$ & $1.5-1.9$ & 1.29 & $\pm 3.2 \%$ & $\pm 0.5 \%$ & $\pm 3.2 \%$ \\
\hline${ }^{58} \mathrm{Ni}(\mathrm{n}, \mathrm{p}) / \mathrm{U} 8$ & $1.5-2.2$ & 0.992 & $\pm 2.1 \%$ & $\pm 0.7 \%$ & $\pm 2.2 \%$ \\
\hline${ }^{54} \mathrm{Fe}(n, p) / U 8$ & $1.5-2.4$ & 1.047 & $\pm 3.0 \%$ & $\pm 0.8 \%$ & $\pm 3.1 \%$ \\
\hline${ }^{46} T i(n, p) / 48$ & $1.5-3.8$ & 0.986 & $\pm 3.2 \%$ & $\pm 1.7 \%$ & $\pm 3.6 \%$ \\
\hline${ }^{63} \mathrm{Cu}(n, \alpha) / \mathrm{U} 8$ & $1.5-4.9$ & 1.129 & $\pm 3.6 \%$ & $\pm 3.4 \%$ & $\pm 5.0 \%$ \\
\hline${ }^{56} \mathrm{Fe}(\mathrm{n}, \mathrm{p}) / \mathrm{U} 8$ & $1.5-6.0$ & 1.013 & $\pm 3.2 \%$ & $\pm 3.0 \%$ & $\pm 4.4 \%$ \\
\hline${ }^{48} \mathrm{Ti}(\mathrm{n}, \mathrm{p}) / \mathrm{U} 8$ & $1.5-6.0$ & 1.000 & $\pm 3.2 \%$ & $\pm 4.1 \%$ & $\pm 5.2 \%$ \\
\hline${ }^{27} \mathrm{Al}(n, \alpha) / \mathrm{U} 8$ & $1.5-6.6$ & 1.076 & $\pm 3.2 \%$ & $\pm 4.8 \%$ & $\pm 5.8 \%$ \\
\hline \multicolumn{6}{|c|}{ Non-Threshold Reactions } \\
\hline $\mathrm{U} 8 /{ }^{239} \mathrm{Pu}(\mathrm{n}, \mathrm{f})$ & $\begin{array}{l}0.3-1.5 \text { low } \\
5.7-7.2 \underline{\text { high }}\end{array}$ & 0.981 & $\pm 1.2 \%$ & $\pm 0.8 \%$ & $\pm 1.4 \%$ \\
\hline$U 8 / 235 U(n, f)$ & $\begin{array}{l}0.22-1.5 \text { low } \\
5.2-6.7 \text { high }\end{array}$ & 0.946 & $\pm 1.2 \%$ & $\pm 1.0 \%$ & $\pm 1.6 \%$ \\
\hline$U 8 / 233 U(n, f)$ & $\begin{array}{l}0.23-1.5 \text { low } \\
5.5-7.2 \underline{\text { high }}\end{array}$ & 0.957 & $\pm 2.1 \%$ & $\pm 1.0 \%$ & $\pm 2.3 \%$ \\
\hline $\mathrm{U} 8 / 241 \mathrm{Pu}(\mathrm{n}, \mathrm{f})$ & $\begin{array}{l}0.22-1.5 \text { low } \\
5.6-7.2 \text { high }\end{array}$ & 0.975 & $\pm 5.7 \%$ & $\pm 1.0 \%$ & $\pm 5.8 \%$ \\
\hline $\mathrm{U} 8 /{ }^{197} \mathrm{Au}(\mathrm{n}, \gamma)$ & $0.06-1.5 \frac{\mathrm{low}}{\underline{\text { high }}}$ & 0.974 & $\pm 3.2 \%$ & $\pm 2.7 \%$ & $\pm 4.2 \%$ \\
\hline
\end{tabular}

(a) Spectral indexes are taken with respect to the $238 \mathrm{U}(\mathrm{n}, \mathrm{f})$ reaction labeled "U8" with the lower energy response detector in the denominator. The calculated-toobserved ratio for $\bar{\sigma}_{f}($ U238) is 0.962 from Table $X-10$ (B5).

(b) Non-overl apping energy response interval between the $95 \%$ response energy boundary for each detector. Al so see footnote (b) Table $X-11$. For non-threshold reactions, two non-overlapping energy intervals are listed: The $95 \%$ exclusion interval is labeled "low" and the 5\% exclusion interval "high". See Table $X-16(B 5)$.

(c) Errors in the calculated value correspond to ${ }^{252} \mathrm{Cf}$ fission spectrum uncertainties only, given in Table $X-5$, and propagated according to the second term of Eq. $(X-8)$, Section 5.a.3. The error in $\bar{\sigma}_{f}(U 238)$ calculated is $\pm 0.8 \%$ (10) from Table $X-10(B 5)$; error in the observed value is from Table $X-11$. 


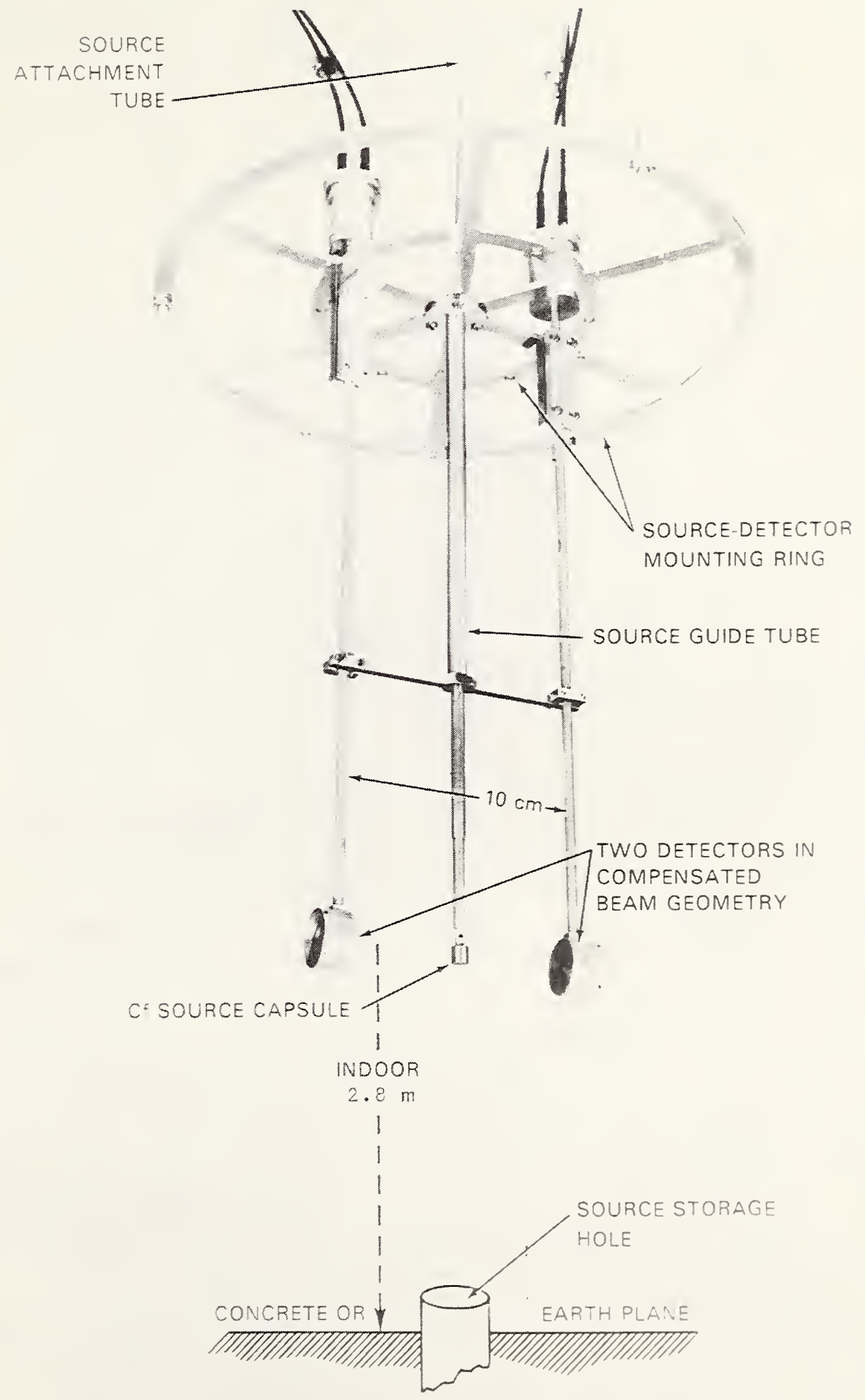

Figure $x-1 . \quad{ }^{252} \mathrm{Cf}$ Fission Spectrum Irradiation Facility at ies 


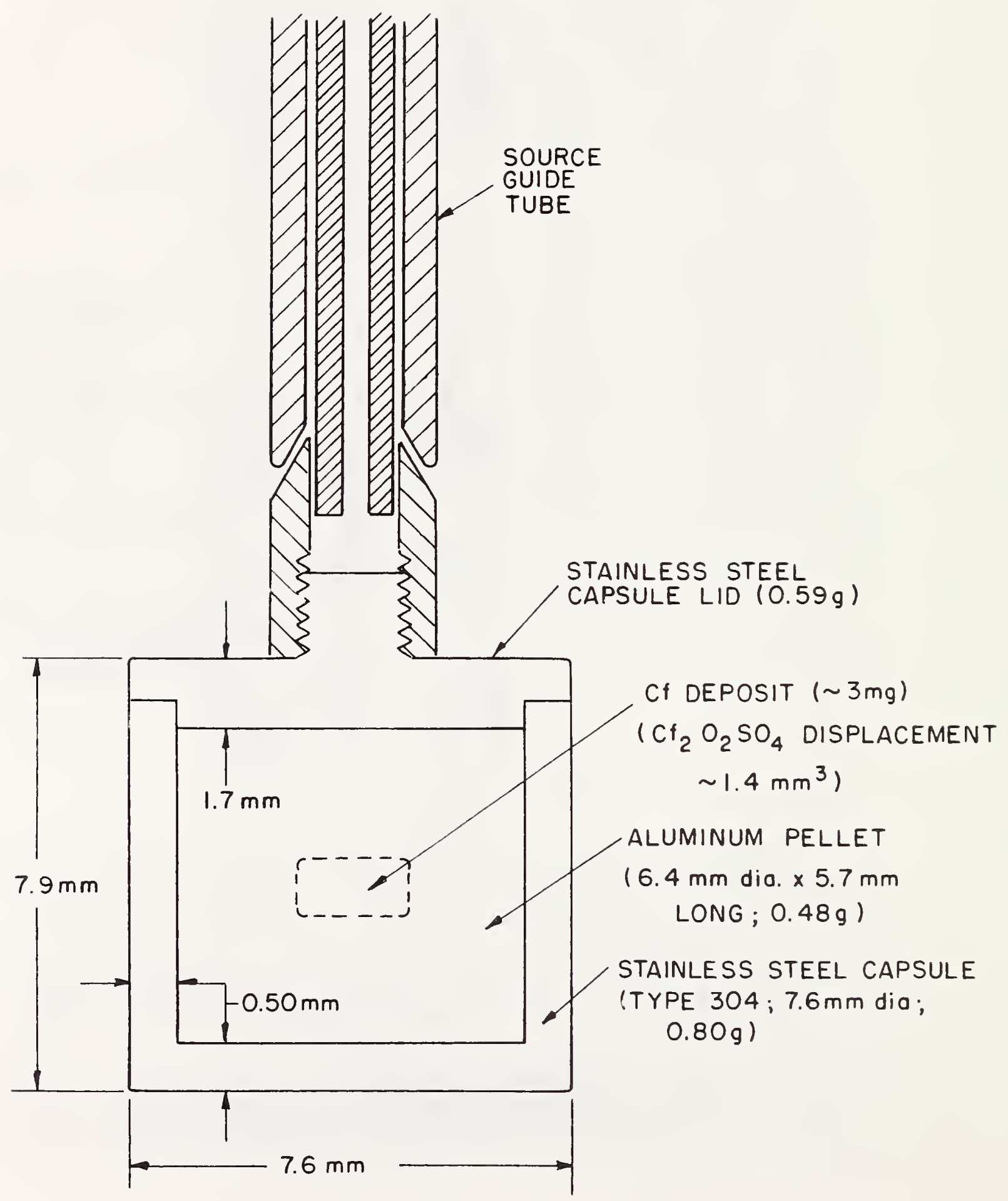

Figure X-2. $\quad{ }^{252}$ Cf Fission Neutron Cource Capsule and Attachments. 


Part IB: $235 \mathrm{U}$ Thermal-Neutron-Induced Fission Neutron Spectrum 


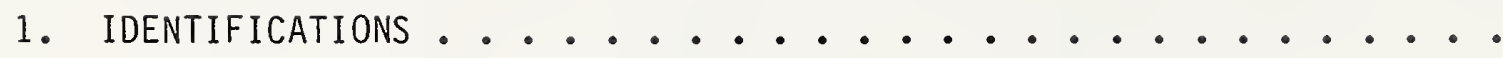

1.a. Title, Classification

1.b. Designation

1.c. Entry Dates and Revisions

1.d. Generic Description

1.e. Facility Locations

1.f. Contacts for Information

2. SUMMARY INFORMATION ............................ 44

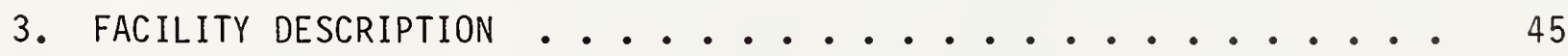

3.a. Configuration and Characteristics

(location, fluence rate, scattering)

3.b. Irradiation Procedures

(experimental arrangements, availability, fluence monitoring)

3.c. Specification For Transport Calculations

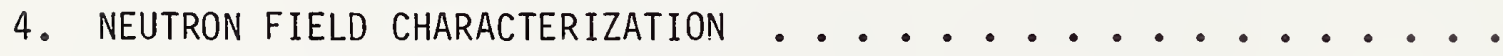

4.a. Neutron Fluence

4.b. Neutron Spectrum

(intensity, gradients, and scattering perturbations)

4.b.1. Calculation

4.b.2. Measurements

4.b.3. Evaluated Spectrum

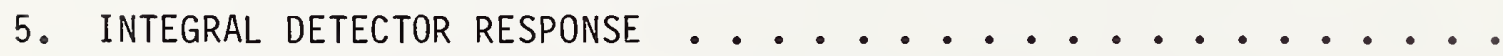

5.a. Calculated Reaction Probabilities

(formulation, truncation, spectrum fraction)

5.a.1. Spectrum Response Table

(cross sections, spectrum fraction, response range)

5.a.2. Spectral Indexes

5.b. Measured Reaction Probabilities

5.c. Measured Cross Sections and Calculated-to-Observed Ratios

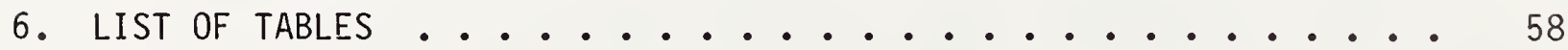

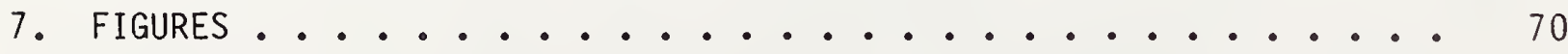




\section{IDENTIFICATIONS}

1.a. NEUTRON FIELD: 235U THERMAL-NEUTRON-INDUCED FISSION SPECTRUM CLASSIFICATION: STANDARD NEUTRON FIELD

1.b. DESIGNATIONS: XU5-5-N7 for NBS spectrum evaluation [Gr75b], [Gr75c] XU5-9-E5 for ENDF/B-V spectrum evaluation

1.C. ENTRY DATE: June, 1978 REVISIONS: JuTy, 1985 July, 1983

\section{1.d. GENERIC DESCRIPTION}

The standard spectrum consists of neutrons from the thermal-neutron-induced fission of $235 \mathrm{~J}$. The median energy of the spectrum is $1.57 \mathrm{MeV}$ with $98 \%$ of the spectrum between 0.1 and $7 \mathrm{MeV}$. Contributions from scattered neutrons and other backgrounds must be small and well defined if a particular $235 \mathrm{U}$ fission source facility is to qualify as a standard neutron field. Fission neutron fluence rates of up to $\sim 4 \times 10^{10} \mathrm{n} /\left(\mathrm{cm}^{2} \mathrm{~s}\right)$ have been generated in reactor thermal-columns with spherical cavities. Source to detector distances are small in such arrangements and the pure fission neutron field is restricted to a volume of one to ten cubic centimeters.

Extensive and well documented measurements exist for the $235 \mathrm{U}$ fission neutron spectrum and its $\mathrm{cl}$ ose relative the ${ }^{252} \mathrm{Cf}$ fission spectrum. Therefore these two standard fields are much better known than any other benchmark employed for reactor dosimetry calibration. In the energy range above $2 \mathrm{MeV}$, many neutron fields in and around test and power reactors have fission-spectrumlike components. 
1.e. FACILITY LOCATIONS:

National Bureau of Standards

Center for Radiation Research

Gaithersburg, Md 20899

U.S.A.

Contact: Dale McGarry or George Lamaze

Phone: 301-921-2767
Research Reactor Institute Kyoto University

Kyoto, Japan

Contact: Itsuro Kimura

Centre D'Etude De L'Energie Nucleaire

SCK/CEN

BOERETANG 200

B-2400 MOL

Bel gi um

Contact: Albert Fabry or Reactor Physics Section

Phone: (011) 3214311801

1.f. CONTACT FOR INFORMATION:

Dale McGarry or James Grundl

Center for Radiation Research

National Bureau of Standards

Gaithersburg, Maryl and 20899

U.S.A.

Phone: 301-921-2767

\section{SUMMARY INFORMATION}

2.a. AVAILABLE FISSION NEUTRON EXPOSURES (NBS ONLY)

- fluence rate

- nominar maximum ffuence

- accuracy of free-field fluence $\sim 3.5 \times 10^{10 \mathrm{n} /\left(\mathrm{cm}^{2} \mathrm{~s}\right)}$

$5 \times 10^{15} \mathrm{n} / \mathrm{cm}^{2}$

$\pm 2.3 \%(1 \sigma)$

2.b. CORRECTIONS FOR NEUTRON SCATTERING IN SOURCE AND ENCAPSULATIONS (NBS ONLY)

- neutron ffuence

- neutron reactions with threshold $<3 \mathrm{MeV}$

$(0.0$ to +0.6$) \%$

- neutron reactions with threshold > $3 \mathrm{MeV}$

$(0.0$ to -1.0$) \%$ 


\section{FACILITY DESCRIPTION}

Fission neutron sources in spherical cavities are in operation at two reactor thermal-column facilities. At SCK/CEN in Belgium a one meter diameter spherical cavity in graphite provides fission neutron fluences in a cyl inder-source arrangement which allows low-energy background components to be established experimentally [Fa74a], [Fa75b], [Fa82a], [Gi84a]. The source and detector access geometry, and its location in a reactor thermal column, are shown in Fig. X-4. At NBS in the United States, a 30-cm diameter spherical cavity generates a higher fission neutron fluence than is available at the SCK/CEN facility. However, because of the smaller diameter cavity and a disk-shaped fission source, low-energy backgrounds are more difficult to estimate in the NBS Facility [Fa72b], [Gr72a], [Mc82a], [Mc84a]. At Kyoto University, a high-

and low-power fission plate converter system is enclosed in a cubical concrete shield with $\sim 2.5 \mathrm{~m}$ wall-to-wall separation [Ki78a]. Only the NBS facility will be described in this compendium.

\section{3.a. CONFIGURATION AND CHARACTERISTICS [Mc82a], [Mc84a], [La82a]}

The NBS cavity fission source operates at the center of a $30 \mathrm{~cm}$ diameter spherical cavity in the NBS Research Reactor graphite thermal column. The source-detector capsule consists of two coaxial source disks of $235 \mathrm{U}$ metal (16 mm dia $\times 0.13 \mathrm{~mm}$ thick) placed outside a cadmium box that encloses passive detectors for exposure. The cavity and source-detector arrangement are shown in Fig $X-3$. The source-detector assembly is designed so that the cadmium box and detectors may be removed separately from the fission disks. Fission neutron fluence rates of $\sim 4 \times 10^{10} \mathrm{n} / \mathrm{cm}^{2} \mathrm{~s}$ are obtained at the midplane between the source disks when they are at a separation distance of $11 \mathrm{~mm}$. For 
thin disk-shaped detectors with diameters up to $13 \mathrm{~mm}$, axial fluence gradients are mild: the fluence at $1 \mathrm{~mm}$ from the midplane is $4 \%$ greater than at the midplane. Center-to-edge fluence ratios are more severe - see Section $4 a$.

Fission neutron return for spherical cavities in graphite has been studied extensively by means of neutron transport calculations and by experiment in connection with the general development of standard neutron fields in spherical geometry $[\mathrm{Ei77a}],[\mathrm{Fa} 72 \mathrm{~b}],[\mathrm{Fa} 75 \mathrm{C}],[\mathrm{Fa} 82 \mathrm{a}],[\mathrm{Gr} 62 \mathrm{a}]$. For the 30-cm cavity at NBS, the response of threshold fission detectors to fission neutrons returning from the cavity walls is less than $0.3 \%$ of the uncollided fission neutron fluence from the fission source disks; for detectors with thresholds above $1 \mathrm{MeV}$, the response to the cavity return fluence is less than $0.1 \%$. These estimates, based on calculation, have been checked experimentally by means of fission chamber traverses in the cavity fission sources at SCK/CEN [Fa82a]. More important is fission neutron scattering in the source-detector capsule. Monte Carlo calculations are employed for this problem and they provide corrections for threshold detectors which vary with detection threshold and with the number and arrangement of detector disks [Mc82a]. Corrections for activation rates are generally between $0.5 \%$ and $3 \%$.

The NBS fission source is not used generally for low-energy integral detectors because of uncertainties in the graphite return background. The graphite return correction for the $235 \mathrm{U}(n, f)$ reaction, for example, is nearly $10 \%$ and self-shielding of the near-1/E cavity return fluence by the fission disks and by a $235 \mathrm{U}$ activation foil makes this correction uncertain to a factor of about one third. The one meter cavity arrangement at SCK/CEN is better suited for low-energy response detectors [Fa82a]. 


\section{3.b. IRRADIATION PROCEDURES:}

Disk detector irradiations are monitored with nickel or indium monitor foils placed between the detectors. The neutron fluence in the detector capsule is obtained from the measured ${ }^{58} \mathrm{Ni}(n, p)$ or ${ }^{115} \operatorname{In}\left(n, n^{\prime}\right)$ activity on the basis of a fluence transfer calibration carried out at the NBS ${ }^{252} \mathrm{Cf}$ Irradiation Facility. In this procedure, the ratio of the activity obtained in a known ${ }^{252} \mathrm{Cf}$ fission neutron fluence to that obtained during a cavity fission source irradiation is translated into a $235 \mathrm{U}$ fission neutron fluence at each monitor foil position in the source-detector capsule.

The expression which governs this fluence transfer is

$$
\begin{aligned}
\Phi_{C v y} & =\frac{R_{c v y}}{R_{c f}} \cdot \frac{\bar{\sigma}_{c f}}{\bar{\sigma}_{c v y}} \Phi_{C f} \\
& =\frac{G_{c f}}{G_{c v y}} \cdot \frac{\sigma_{c f}\left(>E_{p}\right)}{\sigma_{c v y}\left(>E_{p}\right)} \cdot \frac{\psi_{c f}\left(>E_{p}\right)}{\psi_{c v y}\left(>E_{p}\right)} \cdot \frac{D_{c v y}}{D_{c f}} \cdot \Phi_{c f}
\end{aligned}
$$

where $\Phi=$ fission neutron fluence and "cvy" denotes the $235 \mathrm{U}$ cavity fission source and "cf" the ${ }^{252} \mathrm{Cf}$ fission neutron calibration fluence,

$$
E_{p}=\text { truncation energy }(p=0.95 \text { commonty }) \text {. }
$$

The ratios on the right side of the equation involve decay correction factors $(G)$, truncated reaction cross sections $\sigma\left(>E_{p}\right)$, spectrum fractions $\psi\left(>E_{p}\right)$, and the ratio of the monitor foil activity in the cavity to that at the ${ }^{252} \mathrm{Cf}$ irradiation facility - see Section 5.a in Part IA. Details of the fluence transfer procedure are given in Refs. [Mc84a], [La84a]. These references also describe a set of validation experiments, carried out at the SCK/CEN Facility,

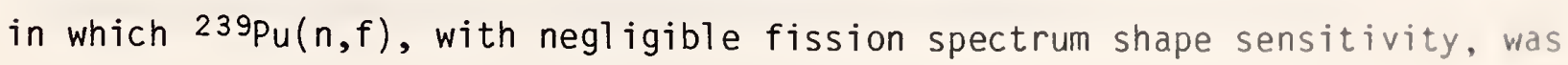


effectively substituted for ${ }^{58} \mathrm{Ni}(n, p)$ and ${ }^{115} \operatorname{In}\left(n, n^{\prime}\right)$ as the fluence transfer reaction. The fluence transfer procedure yields a free-field $235 \mathrm{U}$ cavity fission neutron fluence to an accuracy of $\pm 2.3 \%(1 \sigma)$.

Fission neutron fluences of $\sim 3 \times 10^{15} \mathrm{n} / \mathrm{cm}^{2}$ may be obtained at the NBS facility in 24 hour long irradiations. The small irradiation volume restricts multiple foil packages to a diameter of $12 \mathrm{~mm}$ and a thickness of a few millimeters. The gamma-ray dose from neutron capture in graphite $(\sim 0.7 \mathrm{kGy} / \mathrm{h})$ and cadmium $(\sim 4.4 \mathrm{kGy} / \mathrm{h})$ is about $46 \%$ of the neutron and gamma dose from the fission sources.

\section{C. SPECIFICATIONS FOR TRANSPORT CALCULATIONS}

Neutron transport calculations are required for two types of corrections:

(1) neutron scattering in the fission source disks and in the detector capsule; and (2) neutron return from the graphite.

The graphite thermal column at the NBS Reactor is effectively infinite for fission neutrons originating in the cavity. Graphite density is $1.74 \mathrm{~g} / \mathrm{cm}^{3}$, and the cavity is a well formed sphere $29.8 \mathrm{~cm}$ in diameter. The two fission source disks may be considered as a point source at the cavity center.

Scattering in the source-detector capsule has been established on the basis of Monte Carlo calculations [Mc82a]. The main scattering components in these calculations (see Fig. $X-3$ ) are the cadmium box (22.5-mm dia., 8.3-mm high, and $0.076-\mathrm{mm}$ thick), the source disks (16-mm dia. $\times 0.13-\mathrm{mm}$ thick), and the foil samples (typically less than $2 \mathrm{~g}$ ). Although the nickel and indium fluence monitor foils are in the same geometry as the foil samples, they do not exactly monitor energy-angle effects for all types of threshold detectors. The latter effect, along with energy transfer by inelastic scattering, are the 1 argest uncertainties in detector response corrections for neutron scattering. 


\section{NEUTRON FIELD CHARACTERIZATION}

The energy spectra of fission neutrons are very similar for all fissionable materials and both test and power reactors exhibit fission-spectrum-like components in the MeV energy range. Using the same description as in Part IA, the $235 \mathrm{U}$ fission neutron spectrum may be described in terms of a broad energy range,

\begin{tabular}{|c|c|c|c|}
\hline & $\begin{array}{r}1 \text { lower bound } \\
E_{p}(p=0.99)\end{array}$ & $\begin{array}{c}\text { median } \\
E_{p}(p=0.5)\end{array}$ & $\begin{array}{l}\text { upper bounc } \\
E_{p}(p=0.01)\end{array}$ \\
\hline 35 & $0.08 \mathrm{MeV}$ & $1.57 \mathrm{MeV}$ & $7.2 \mathrm{MeV}$ \\
\hline
\end{tabular}

and a coarse seven-group flux display, $\phi(E) \Delta E$, as follows:

$\begin{array}{llllllll}0 & 0.25 & 0.8 & 1.5 & 2.3 & 3.7 & 8 & 12 \mathrm{MeV}\end{array}$
$235 \mathrm{U}$
$(0.054)$
0.197
0.229
0.195
0.192
0.127
0.006

A detailed description of the spectrum is given in Section 4.b.

Although the neutron spectrum 1 isted is specifically for thermal-neutroninduced $235 \mathrm{U}$ fission, the fission spectrum for fast-neutron-induced fission is little different. The change in median energy is about $0.05 \mathrm{MeV}$.

4.a. NEUTRON FLUENCE (see also Sections 3.a. and 3.b.)

The combined source strength for the two fission disks in the thermal column cavity is approximately $3 \times 10^{11} \mathrm{n} / \mathrm{s}$. Since this source strength is difficult to determine accurately, the $235 \mathrm{U}$ fission neutron fluence is established by 
means of fluence transfer from the ${ }^{252} \mathrm{Cf}$ fission neutron irradiation facil ity to an accuracy of $\pm 2.3 \%$, exclusive of scattering in detectors - see

Section 3.b. The neutron fluence at the midplane of the source-detector capsule for a source disk separation of $12 \mathrm{~mm}$ is $0.12 \mathrm{n} / \mathrm{cm}^{2}$ per source neutron corresponding to a fluence rate of $\sim 3.5 \times 10^{10} \mathrm{n} / \mathrm{cm}^{2} \mathrm{~s}$ with the reactor at 20 megawatts full power. The fission neutron fluence rate is minimum at the midpl ane between the two source disks. At $1 \mathrm{~mm}$ above or below the midplane, the mean fluence for a $12 \mathrm{~mm}$ diameter disk detector is $\sim 4 \%$ higher than at the midplane. Radial variations are more severe: center-to-edge fluence ratios are 1.2 for $12 \mathrm{~mm}$ diameter disks. This gradient does not require that detector disks be extremely uniform. A radial mass nonuniformity of $20 \%$, for example, distributed linearly in a detector foil changes the activation by less than $0.5 \%$ compared to a uniform mass distribution. Also, the 115 In fluence monitor included in each irradiation package yields a fluence averaged over the radial variation.

The fission neutron fluence is perturbed by a small cavity return contribution, and also by scattering in fission source disks, in encapsulations, and in the detector foils. The correction for these effects, based on calculation, is given as the fractional departure for each detector $\left(\mu_{s c}\right)$ from its free-field activity attributable to both neutron scattering and cavity return:

$$
\left[\begin{array}{c}
\text { total } \\
\text { measured } \\
\text { activity }
\end{array}\right]=\left[\begin{array}{c}
\text { free-fiel } d \\
\text { activity }
\end{array}\right]\left[1+\mu_{s c}\right] \text {. }
$$

This scattering correction depends markedly upon reaction threshold because of energy-angle correlation in the nearby materials of the encapsulation. 
Components of $\mu_{S C}$, obtained from detailed Monte-Carlo calculations of the source detector capsule with a representative foil stack, are as follows:

\begin{tabular}{|c|c|c|c|c|}
\hline & $\begin{array}{l}\text { neutron } \\
\text { fl uence }\end{array}$ & $\begin{array}{l}\text { changes in } t \\
E_{95}=1.2 \mathrm{MeV} \\
\end{array}$ & $E_{95}=2.1 \mathrm{MeV}$ & $E_{95}=6.5 \mathrm{MeV}$ \\
\hline cavity return & $+1.2 \%$ & $+0.17 \%$ & $<0.1 \%$ & $<0.1 \%$ \\
\hline $\begin{array}{l}\text { encapsulations } \\
\text { (incl. source } \\
\text { disks and cd box) }\end{array}$ & $+2.5 \%$ & $+(0.5 \pm 0.3) \%$ & $-(0.2 \pm 0.3) \%$ & $-(1.3 \pm 0.3) \%$ \\
\hline foil stack & $+1.5 \%$ & $+(0.0$ to 0.5$) \%$ & $-(0.4$ to 1.0$) \%$ & $-(0.8$ to 1.5$) \%$ \\
\hline$\mu_{S C}(\%):$ & & $+(0.7$ to 1.2$) \%$ & $-(0.6$ to 1.2$) \%$ & $-(2$ to 3$) \%$ \\
\hline
\end{tabular}

The spread of the foil-stack component of $\mu_{s c}$ is associated with foil position. It is possible to derive from the Monte Carlo calculation individual foil-to-foil scattering factors as a function of foil separation. These correction functions $c$ an be applied to a range of foil stack arrangements beyond the one calculated as long as the foil-to-foil scattering is mainly first collision. An overall error of \pm 0.6 is assigned to $\mu_{s c}$ expressed in percent.

It is the small source-to-detector distance which makes it possible to obtain nearly pure fission neutron fluences in small cavities. The cavity return fluence at the cavity center is about $1.2 \%$ of the uncollided fission neutron fluence. This return fluence gives rise to threshold detector responses that are between $0.05 \%$ and $0.3 \%$ of the free-field fission neutron response depending upon the reaction threshold. 


\section{4.b. NEUTRON SPECTRUM}

4.b.1. Calculation. Fission neutron spectra are most accurately determined by laboratory measurement. (See, however, Ref. [Ma82b] and earlier work cited therein.)

4.b.2. Measurements. See Section 4.b.2. in Part $1 A$.

4.b.3. Evaluated Spectrum. The NBS fission spectrum evaluation is chosen for this compendium because of the consistency of its procedures for both ${ }^{235} \mathrm{U}$ and ${ }^{252} \mathrm{Cf}$ fission spectra, and because it includes an articulated error estimate $[G r 75 c],[G r 75 b]$. Cross section comparisons with the ENDF/B-V $235 \mathrm{U}$ fission spectrum shape will be given as a supplement.

The NBS evaluated spectrum is described up to $20 \mathrm{MeV}$ by means of a reference Maxwellian, $M(E)$, modified by four piecewise continuous linear segments below $6 \mathrm{MeV}$ and one exponential segment above $6 \mathrm{MeV}$. The reference Maxwellian for the $235 \mathrm{U}$ fission spectrum is,

$$
M(E)=0.750 \sqrt{E} \exp (-1.5 E / 1.97), E \text { in } M e V,
$$

and the adjustment functions $\mu(E)$ are as follows:

\begin{tabular}{c} 
Energy Interval (MeV) \\
\hline $0-0.25$ \\
$0.25-0.8$ \\
$0.8-1.5$ \\
$1.5-6.0$ \\
$6.0-20$
\end{tabular}

$$
\begin{aligned}
\frac{\mu_{25}(E)}{1+0.8 E-0.153} \\
1-0.14 E+0.082 \\
1+0.040 E-0.062 \\
1+0.01 E-0.017 \\
1.043 \exp [-0.06(E-6.0) / 1.043]
\end{aligned}
$$


The evaluated spectrum is given by $x(E)=\mu(E) \cdot M(E)$. A 45-group tabulation of the evaluated $235 \mathrm{U}$ fission spectrum is given in Table X-4, Part IA. Group fluences for other energy group structures may be derived from this tabulation with the interpolation function recommended in the footnote to the table. Spectrum uncertainties, given in Table X-5 of Part IA, are based on departures of subsets of measured data from the final adjusted Maxwellian of the evaluation. This estimate, carried out in a seven-group structure, includes both $1 \sigma$ and $2 \sigma$ errors.

of the many descriptions employed for the $235 \mathrm{U}$ fission spectrum, the most common is the Watt-function fit chosen for ENDF/B-V in June, 1979:

$$
\begin{gathered}
\psi(E)=C \exp (-E / a) \sinh (\sqrt{\mathrm{bE}}) \\
a=0.988 \mathrm{MeV} \quad \mathrm{b}=2.249 \mathrm{MeV}-1 \quad C=0.4395 \\
\text { median energy: } E_{p}(p=0.5)=1.64 \mathrm{MeV} .
\end{gathered}
$$

Contemporary time-of-f7 ight data was emphasized in the ENDF/B-V evaluation as compared with the NBS eval uation which considered all well-documented data. Differences between the two eval uated spectra, in terms of calculated spectral indexes, are shown in Table $X-14 a(B 5)$ and $X-14 a .1$.

\section{INTEGRAL DETECTOR RESPONSE}

Neutron dosimetry measurements with integral detectors generally benefit from the use of benchmark neutron fields for cal ibrating measurement techniques and for referencing data interpretation methods. In order to carry out these 
measurement assurance procedures, and in particular for estimating uncertainties, it is necessary to distinguish between calculated and measured reaction probabilities and to identify parameters which characterize the energy response of each detector in the various spectra to which it is exposed. A formulation which meets these requirements is described in Section 5 of Part IA. Response parameters for a number of integral detectors, including cross sections, spectral indexes, and energy response ranges, are presented in Section 5.a. Measured reaction probabilities are given in Section 5.b followed by comparisons of measured and calculated cross sections in Section 5.c.

\section{5.a. CALCULATED REACTION PROBABILITIES}

(See Section 5.a in Part IA for reaction probability formulations, parameters, and error propagation.)

5.a.1. Spectrum Response Table. Basic integral detector response parameters for $235 \mathrm{U}$ fission neutrons are given in Table $X-13(B 5)$. The spectrum-averaged cross sections 1 isted in column 2 are the ful1-spectrumaveraged values above a cadmium cut-off energy of $0.4 \mathrm{eV}$ and are followed in column 3 by cross sections truncated at $p=0.95$. The energy dependent cross sections employed are those of the ENDF/B-V dosimetry file reduced to 620 energy groups; spectrum averaging is carried out with the NBS DETAN code. The spectrum fraction, $\left[\psi\left(>E_{p}\right), p=0.95\right]$, follows in column 4. Energy response characteristics, given in the last three columns, are the median response energy and the energy response range, i.e., the lower- and upper-energy bounds that include $90 \%$ of the detector response. The low-energy bound, $E_{p}(p=0.95)$, is the truncation energy corresponding to the truncated cross section given in column 3 . 
5.a.2. Spectral Indexes (See Section 5.a.2, Part IA for formulations).

selected set of spectral indexes calculated for full-spectrum-averaged cross sections from Table $X-13(B 5)$ is given in Table $X-14(B 5)$; a corresponding set for truncated cross sections is in Table $X-15$. All of these spectral indexes are for the NBS evaluated fission spectrum shape described in Section 4.b.3. Tables $14 a(B 5)$ and 14.a.1 list spectral indexes for the ENDF/B-V fission spectrum and compare the two evaluations.

5.b. MEASURED REACTION PROBABILITIES (See Section 5.b, Part IA)

5.C. MEASURED CROSS SECTIONS AND CALCULATED TO OBSERVED RATIOS

For this compendium, the primary integral detector quantities of interest are cross sections and spectral indexes taken with respect to the ${ }^{238} U(n, f)$ reaction. Available integral detector data for the $235 \mathrm{U}$ fission spectrum are to be found in References [Ac81a], [A782a], [Fa74a], [Fa75a], [Fa75b], $[\mathrm{Fl77a}],[\mathrm{Gi} 84 \mathrm{a}],[\mathrm{Gr72a}],[\mathrm{Gr} 75 \mathrm{~b}],[\mathrm{Gr} 83 \mathrm{a}],[\mathrm{Gr} 85 \mathrm{a}],[\mathrm{Ki78a}],[\mathrm{La} 82 \mathrm{a}]$, [Le57a], [Ma84b], and [0182a]. Collections of evaluated measured cross sections may be found in References [Fa72a], [Fa78a], [Gr70a], and [Ha78a].

A new and restricted evaluation of measurements reported before July 1985, was performed at NBS for detector reactions considered important for reactor technology. The evaluation begins with the absolute ${ }^{235} U(n, f)$ and ${ }^{239} \mathrm{Pu}(n, f)$ cross sections measured for ${ }^{252} \mathrm{Cf}$ fission neutrons. Since these cross sections for ${ }^{252} \mathrm{Cf}$ and $235 \mathrm{U}$ fission neutrons are the same to within a conservative upper bound of $\pm 0.6 \%$, cross section measurements performed relative to either one in the ${ }^{235} \mathrm{U}$ fission spectrum can be scaled to the ${ }^{252} \mathrm{Cf}$ cross section value with very little additional error. For most $235 \mathrm{U}$ fission spectrum measurements performed at NBS and SCK/CEN, this fluence transfer technique establishes a fission neutron fluence based upon a ${ }^{252}$ cf neutron 
source strength - see Section 3a, Part IA. For other measurements not so normalized, the ${ }^{115} \operatorname{In}\left(n, n^{\prime}\right)$ or $58_{\mathrm{Ni}}(n, p)$ reactions are construed in the evaluation as the fluence monitors. Results are given in Table X-16 and X-18. The uncertainties given at one standard deviation include estimates of systematic errors.

Calculated-to-observed ratios of reaction cross sections are 1 isted in Table $X-17(B 5)$ and include comparison with both the NBS evaluated and the ENDF/B-V fission spectrum shapes. Errors in the calculated cross section correspond to uncertainties in the fission spectrum (Table $X-5$ ) propagated according to the second term of Eq. $(X-7)$ (Part IA, Section 5.a.3). Only the ${ }^{47} \mathrm{Ti}(n, p)$, ${ }^{48} \mathrm{Ti}(n, p)$, and ${ }^{232} \mathrm{Th}(n, f)$ reactions show calculated-to-observed disagreements in excess of $10 \%$; however, twelve $\mathrm{C} / \mathrm{E}$ ratios are outside of one standard deviation for the NBS evaluated spectrum. Two threshold reactions important for reactor dosimetry, ${ }^{115} \operatorname{In}\left(n, n^{\prime}\right)$ and $58_{N i}(n, p)$, disagree with predicted values by nearly 10\%. Of particular importance for cross section evaluation is the apparent $(8-10) \%$ under prediction of the measured $10 \mathrm{~B}(\mathrm{n}, \mathrm{He})$ reaction cross section by ENDF/B-V. The standard deviation of fifteen $C / E$ ratios in Table $X-17(B 5)$ for the NBS spectrum, excluding ${ }^{47} T i(n, p),{ }^{232} \operatorname{Th}(n, f)$, and 239pu(n,f), are 0.038 about an average departure from unity of 1.062 . The standard deviation for the same fifteen C/E ratios in the ENDF/B-V fission spectrum are 0.029 about an average departure from unity of 1.035 .

Calculated-to-observed ratios of spectral indexes, $\mathrm{C}_{\alpha / \mathrm{U} 8}$, are presented in Table $X-19(B 5)$. The calculated indexes correspond to full-spectrum averaged cross section ratios for the NBS spectrum evaluation from Table $X-14(B 5)$. Contributing errors for observed and calculated indexes are 1 isted separately. 
Errors for the calculated indexes correspond to fission spectrum uncertainties listed in Table $X-5$ propagated according to Eq. $(X-8)$ (Part IA, Section 5.a.3).

The $C_{\alpha / U 8}$ ratios in Table-XI9(B5) validate integral detector measurement techniques and detector cross sections. The difficulties with the Ti isotopes, which was so evident with the ENDF/B-IV dosimetry file, are improved except for $47 \mathrm{Ti}$ where a $27 \%$ discrepancy remains. The $9 \%$ discrepancy for $\mathrm{C}(238 \mathrm{U} / 235 \mathrm{U})$ is consistent with that obtained for ${ }^{252} \mathrm{Cf}$ fission spectrum neutrons (see Table $x-12)$. Of interest for reactor physics is the disagreement of $(4.7 \pm 1.7) \%$ with ENDF/B-V of the spectrum independent $239 \mathrm{pu} / 235 \mathrm{U}$ fission cross section ratio. The corresponding disagreement obtained in the ${ }^{252} \mathrm{Cf}$ spectrum is $(3.7 \pm 1.5) \%$. 
Table $X-13(B 5)$

Integral Detector Response Parameters

$5 . a \cdot 1$

Table $\mathrm{X}-14(\mathrm{B5})$

Calculated Spectral Indexes - NBS Evaluated

Fission Spectrum

$5 . a \cdot 2$

Table X-14a(B5) Calculated Spectral Indexes - ENDF/B-V

Evaluated Fission Spectrum

$5 . \mathrm{a} \cdot 2$

Table X-14.a.1 Calculated Spectral Index Ratios: NBS Evaluation vs. ENDF/B-V Evaluation Fission Spectrum

$5 \cdot a \cdot 2$

Table $X-15(B 5)$

Calculated Spectral Indexes - Truncated

$5 . a \cdot 2$

Table $\mathrm{X}-16$

Observed Integral Cross Sections

$5 . c$

Table $X-17(B 5)$

Calculated-to-Observed Ratios of Cross Sections

$5 . c$

Table $\mathrm{X}-18$

Observed Spectral Indexes

$5 . c$

Table X-19(B5)

Calculated-to-Observed Ratios of Spectral

Indexes - NBS Evaluation

$5 . \mathrm{c}$ 
TABLE $X-13: B 5)$. INTEGRAL DETECTOR RESPONSE PARAMETERS

Spectrum: $235 \mathrm{U}$ Thermal Neutron-Induced Fission

(NBS evaluation, [Gr75b], [Gr75c])

Cross Sections: ENDF/B-V Dosimetry File [EN79a]
Designation: $\quad X U 5-5-N 1$

Entry Date: June, 1978

Revised: March, 1982

\begin{tabular}{|c|c|c|c|c|c|c|}
\hline $\begin{array}{l}\text { Detector } \\
\text { Reaction }\end{array}$ & $\begin{array}{r}\text { Cross S } \\
\sigma(> \\
E_{p}=0.4 e V \\
\end{array}$ & $\begin{array}{l}\text { ction }(a) \\
p(p=0.95) \\
\end{array}$ & $\begin{array}{c}\text { Spectrum }(b) \\
\text { Fraction } \\
\psi\left(>E_{95}\right) \\
\end{array}$ & $\begin{array}{l}\text { Median }(c) \\
\text { Response } \\
\text { Energy } \\
E_{p}(p=0.5) \\
\end{array}$ & $\begin{array}{r}\text { Response } \\
E_{p}(p=0.95)\end{array}$ & $\frac{\text { Range }^{(c)}}{E_{p}(p=0.05)}$ \\
\hline $\begin{array}{l}\frac{\text { Spectrum Check }}{\text { Constant }} \\
\text { recip. vel., (l/v) (d) }\end{array}$ & $\begin{array}{l}\text { (barns) } \\
1.000 \\
0.1151\end{array}$ & $\begin{array}{l}\text { (barns) } \\
1.000 \\
0.1103\end{array}$ & $\begin{array}{l}0.950 \\
0.991\end{array}$ & $\begin{array}{l}(\mathrm{MeV}) \\
1.57 \\
0.921\end{array}$ & $\begin{array}{l}(\mathrm{MeV}) \\
0.24 \\
0.076\end{array}$ & $\begin{array}{l}(\mathrm{MeV}) \\
5.1 \\
4.0\end{array}$ \\
\hline $\begin{array}{c}\text { Fissionable Mat'1s } \\
239 \mathrm{Pu}(\mathrm{n}, \mathrm{f}) \\
235 \mathrm{U}(\mathrm{n}, \mathrm{f})\end{array}$ & $\begin{array}{l}1.786 \\
1.236\end{array}$ & $\begin{array}{l}1.800 \\
1.221\end{array}$ & $\begin{array}{l}0.9424 \\
0.962\end{array}$ & $\begin{array}{l}1.65 \\
1.57\end{array}$ & $\begin{array}{l}0.26 \\
0.20\end{array}$ & $\begin{array}{l}5.2 \\
5.2\end{array}$ \\
\hline $\begin{array}{l}233 U(n, f) \\
238 U(n, f)\end{array}$ & $\begin{array}{l}1.912 \\
0.2947\end{array}$ & $\begin{array}{l}1.895 \\
0.532\end{array}$ & $\begin{array}{l}0.959 \\
0.527\end{array}$ & $\begin{array}{l}1.51 \\
2.67\end{array}$ & $\begin{array}{l}0.21 \\
1.48\end{array}$ & $\begin{array}{l}5.0 \\
6.7\end{array}$ \\
\hline $\begin{array}{l}237 \mathrm{~Np}(n, f) \\
232 \operatorname{Th}(n, f)\end{array}$ & $\begin{array}{l}1.322 \\
0.0724\end{array}$ & $\begin{array}{l}1.578 \\
0.1322\end{array}$ & $\begin{array}{l}0.7958 \\
0.520\end{array}$ & $\begin{array}{l}1.96 \\
2.86\end{array}$ & $\begin{array}{l}0.670 \\
1.50\end{array}$ & $\begin{array}{l}5.7 \\
7.3\end{array}$ \\
\hline $\begin{array}{l}240 p u(n, f) \\
241 P u(n, f)\end{array}$ & $\begin{array}{l}1.326 \\
1.600\end{array}$ & $\begin{array}{l}1.585 \\
1.579\end{array}$ & $\begin{array}{l}0.795 \\
0.963\end{array}$ & $\begin{array}{l}1.97 \\
1.53\end{array}$ & $\begin{array}{l}0.67 \\
0.19\end{array}$ & $\begin{array}{l}5.6 \\
5.1\end{array}$ \\
\hline $\begin{array}{l}238 U(n, \gamma) \\
232 \operatorname{Th}(n, \gamma)\end{array}$ & $\begin{array}{l}0.0721 \\
0.0942\end{array}$ & $\begin{array}{l}0.0691 \\
0.0906\end{array}$ & $\begin{array}{l}0.991 \\
0.988\end{array}$ & $\begin{array}{l}0.87 \\
0.88\end{array}$ & $\begin{array}{l}0.075 \\
0.094\end{array}$ & $\begin{array}{l}2.7 \\
2.8\end{array}$ \\
\hline $\begin{array}{c}\text { Capture Reactions } \\
{ }^{33} \mathrm{Na} \\
{ }^{45} \mathrm{SC} \\
58 \mathrm{Fe}\end{array}$ & $\begin{array}{ll}2.817 & E-4 \\
5.64 & E-3 \\
1.712 & E-3\end{array}$ & $\begin{array}{l}2.69 E-4 \\
5.37 E-3 \\
1.634 \quad E-3\end{array}$ & $\begin{array}{l}0.995 \\
0.997 \\
0.995\end{array}$ & $\begin{array}{l}0.845 \\
0.61 \\
0.948\end{array}$ & $\begin{array}{l}0.053 \\
0.035 \\
0.049\end{array}$ & $\begin{array}{l}4.3 \\
2.7 \\
4.5\end{array}$ \\
\hline $\begin{array}{l}{ }^{59} \mathrm{Co} \\
{ }^{63} \mathrm{Cu}\end{array}$ & $\begin{array}{l}6.28 E-3 \\
1.008 E-2\end{array}$ & $\begin{array}{l}6.00 \quad E-3 \\
9.62 E-3\end{array}$ & $\begin{array}{l}0.995 \\
0.995\end{array}$ & $\begin{array}{l}1.024 \\
0.917\end{array}$ & $\begin{array}{l}0.054 \\
0.055\end{array}$ & $\begin{array}{l}3.8 \\
3.8\end{array}$ \\
\hline $\begin{array}{l}115 \mathrm{In} \\
197 \mathrm{Au}\end{array}$ & $\begin{array}{l}0.1266 \\
0.0810\end{array}$ & $\begin{array}{l}0.1220 \\
0.0774\end{array}$ & $\begin{array}{l}0.986 \\
0.994\end{array}$ & $\begin{array}{l}1.080 \\
0.695\end{array}$ & $\begin{array}{l}0.102 \\
0.058\end{array}$ & $\begin{array}{l}2.9 \\
2.8\end{array}$ \\
\hline
\end{tabular}


TABLE $X-13(B 5)$. INTEGRAL DETECTOR RESPONSE PARAMETERS (Continued)

Spectrum: $235 \mathrm{U}$ Thermal Neutron-Induced Fission

(NBS evaluation, [Gr75b], [Gr75c])

Cross Sections: ENDF/B-V Dosimetry File [EN79a]
Designation: $\quad X U 5-5-N 1$

Entry Date: June, 1978

Revised: March, 1982

\begin{tabular}{|c|c|c|c|c|c|c|}
\hline \multirow[t]{2}{*}{$\begin{array}{l}\text { Detector } \\
\text { Reaction }\end{array}$} & \multicolumn{2}{|c|}{$\begin{array}{c}\text { Cross } \operatorname{Section}(a) \\
\sigma\left(>E_{p}\right)\end{array}$} & \multirow{2}{*}{$\begin{array}{l}\text { Spectrum }(b) \\
\text { Fraction } \\
\psi\left(>E_{95}\right)\end{array}$} & \multirow{2}{*}{$\begin{array}{l}\text { Median }(c) \\
\text { Response } \\
\text { Energy } \\
E_{p}(p=0.5)\end{array}$} & \multirow{2}{*}{$\frac{\text { Response }}{E_{p}(p=0.95)}$} & \multirow{2}{*}{$\frac{\text { Range }^{(c)}}{E_{p}(p=0.05)}$} \\
\hline & $E_{p}=0.4 e V$ & $E_{p}(p=0.95)$ & & & & \\
\hline Helium Production & (barns) & (barns) & & $(\mathrm{MeV})$ & $(\mathrm{MeV})$ & $(\mathrm{MeV})$ \\
\hline${ }^{10} B(n, \alpha)$ & 0.499 & 0.478 & 0.993 & 1.16 & 0.066 & 5.2 \\
\hline${ }^{6} \operatorname{Li}(n, \alpha)$ & 0.465 & 0.455 & 0.971 & 1.25 & 0.167 & 5.7 \\
\hline Threshold Reactions & & & & & & \\
\hline${ }^{115} \operatorname{In}\left(n, n^{\prime}\right)$ & 0.1734 & 0.264 & 0.624 & 2.6 & 1.16 & 5.9 \\
\hline${ }^{47} \mathrm{Ti}(\mathrm{n}, \mathrm{p})$ & 0.02159 & 0.0487 & 0.421 & 3.8 & 1.87 & 7.5 \\
\hline${ }^{32} S(n, p)$ & 0.0676 & 0.198 & 0.324 & 4.0 & 2.3 & 7.4 \\
\hline${ }^{58} \mathrm{Ni}(n, p)$ & 0.1009 & 0.2645 & 0.362 & 4.1 & 2.1 & 7.5 \\
\hline${ }^{54} \mathrm{Fe}(n, p)$ & 0.0778 & 0.2334 & 0.317 & 4.2 & 2.3 & 7.6 \\
\hline${ }^{46} \mathrm{Ti}(\mathrm{n}, \mathrm{p})$ & 0.01081 & 0.0806 & 0.1274 & 5.7 & 3.8 & 9.3 \\
\hline${ }^{27} \mathrm{Al}(n, p)$ & $4.121 E-3$ & $2.522 E-2$ & 0.1553 & 5.8 & 3.5 & 9.3 \\
\hline${ }^{56} \mathrm{Fe}(n, p)$ & $1.006 \mathrm{E}-3$ & $2.446 \mathrm{E}-2$ & 0.0391 & 7.3 & 5.5 & 11.2 \\
\hline${ }^{63} \mathrm{Cu}(n, \alpha)$ & $5.40 E-4$ & $7.80 \mathrm{E}-3$ & $6.58 \mathrm{E}-2$ & 7.3 & 4.7 & 11.1 \\
\hline${ }^{27} A \ell(n, \alpha)$ & $6.93 E-4$ & $3.54 E-2$ & $1.861 \mathrm{E}-2$ & 8.4 & 6.5 & 11.9 \\
\hline${ }^{48} \mathrm{Ti}(\mathrm{n}, \mathrm{p})$ & $2.726 \quad E-4$ & $9.21 E-3$ & $2.813 \mathrm{E}-2$ & 8.1 & 5.9 & 12.2 \\
\hline Additions (e) & & & & & & \\
\hline $103 R h\left(n, n^{\prime}\right)$ & 0.682 & 0.835 & 0.776 & 2.3 & 0.72 & 5.7 \\
\hline${ }^{93} \mathrm{Nb}\left(n, n^{\prime}\right)$ & 0.1543 & 0.2159 & 0.679 & 2.5 & 0.99 & 5.6 \\
\hline${ }^{60} \mathrm{Ni}(\mathrm{n}, \mathrm{p})$ & $2.528 E-3$ & $3.99 \quad E-2$ & 0.0603 & 7.0 & 4.9 & 10.4 \\
\hline $55 \operatorname{Mn}(n, 2 n)$ & 0.2016 E-3 & 0.366 & $0.523 \mathrm{E}-3$ & 12.7 & 11.1 & 16 \\
\hline dpa & 854 & 973 & 0.83 & 2.5 & 0.57 & 6.3 \\
\hline
\end{tabular}


(a) The value given in column 2 is the full-spectrum-averaged cross section above a cadmium cut-off of $0.4 \mathrm{eV}$. The truncated cross section in column 3 is for a truncation energy(column 6) above which $95 \%$ of the detector response occurs. A spectrum-averaged cross section truncated at energy $E_{p}$ is given by

$$
\sigma\left(>E_{p}\right)=\int_{E_{p}}^{\infty} \sigma(E) \psi(E) d E / \int_{E_{p}}^{\infty} \psi(E) d E
$$

(b) The fraction of the spectrum above $E_{95} \equiv E_{p}(p=0.95): \quad \psi\left(>E_{95}\right)=$ $\int_{E_{95}}^{\infty} \psi(E) d E$. The full-spectrum-averaged cross section $\sigma(>0.4 \mathrm{eV})$ is equal
to $\sigma\left(>E_{95}\right) \cdot \psi\left(>E_{95}\right) / 0.95$.

(c) The fractions $p=0.95,0.5$, and 0.05 define energies above which $95 \%, 50 \%$ (median), and $5 \%$ of the detector response occurs, respectively. $E_{p}$ is defined by the relation

$$
\int_{E_{p}}^{\infty} \sigma(E) \psi(E) d E=p \cdot[\sigma(>0.4 \mathrm{eV})]
$$

where $E_{p}(p=1)=0.4 \mathrm{eV}$, and $E(p=0)=20 \mathrm{MeV}$; and $\int_{0.4 \mathrm{eV}}^{\infty} \psi(E) \mathrm{dE}=1$.

(d) Normalization is $\int^{18 \mathrm{MeV}} \sigma_{1 / \mathrm{v}} \mathrm{E} \psi(\mathrm{E}) \mathrm{dE}=1$.

$0.4 \mathrm{eV}$
(e) Cross sections not taken from the ENDF/BV Dosimetry File:

- $\mathrm{Nb}\left(n, n^{\prime}\right)$ is from the IRDF Dosimetry File [Cu80a]

- $\operatorname{Rh}\left(n, n^{\prime}\right)$ is from data reported in Ref. [Pa80a].

- dpa, the atom displacement cross section, is from ASTM Standard Practice E693-79 [An83a]. 
TABLE $X-14(B 5)$. CALCULATED SPECTRAL INDEXES-NBS EVALUATED FISSION SPECTRUM

Spectrum: $235 \mathrm{U}$ Thermal Neutron-Induced Fission (NBS evaluation, [Gr75b], [Gr75c])

Spectral Index: Fu11-Spectrum Cross Section from Tab7e $X-13(B 5)$

(ENDF/B-V Dosimetry File)
Designation: $\quad X U 5-5-N 1$

Entry Date: June, 1978

Revised: March, 1982

$$
\left[\mathrm{S}_{\alpha / \beta}\right]_{\text {calc. }} \equiv\left[\bar{\sigma}_{\alpha} / \bar{\sigma}_{\beta}\right]_{c a l c .}
$$

\begin{tabular}{|c|c|c|c|c|c|}
\hline${ }^{\beta}$ & $\begin{array}{l}235 U(n, f) \\
\sigma=1.236 b)\end{array}$ & $\begin{array}{c}238 U(n, f) \\
(\bar{\sigma}=0.2947 b)\end{array}$ & & $\begin{array}{l}235 U(n, f) \\
\bar{\sigma}=1.236 b)\end{array}$ & $\begin{array}{l}238 U(n, f) \\
(\bar{\sigma}=0.2947 b)\end{array}$ \\
\hline Fission & & & Threshold & & \\
\hline $239 \mathrm{Pu}$ & 1.445 & 6.06 & ${ }^{115} \operatorname{In}\left(n, n^{\prime}\right)$ & 0.1403 & 0.588 \\
\hline $235 U$ & 1.000 & 4.19 & ${ }^{47} T i(n, p)$ & 0.01747 & 0.0733 \\
\hline $233 \mathrm{U}$ & 1.547 & 6.49 & ${ }^{58} \mathrm{Ni}(n, p)$ & 0.0816 & 0.342 \\
\hline $238 \mathrm{U}$ & 0.2384 & 1.000 & ${ }^{54} \mathrm{Fe}(n, p)$ & 0.0630 & 0.2640 \\
\hline $237 \mathrm{~Np}$ & 1.070 & 4.49 & ${ }^{46} T i(n, p)$ & $8.75 E-3$ & 0.0367 \\
\hline $240 \mathrm{Pu}$ & 1.073 & 4.50 & ${ }^{56} \mathrm{Fe}(n, p)$ & $8.14 \mathrm{E}-4$ & $3.41 E-3$ \\
\hline He Prod. & & & ${ }^{63} \mathrm{Cu}(n, \alpha)$ & $4.37 \quad E-4$ & 1.832 E-3 \\
\hline${ }^{10} B(n, \alpha)$ & 0.404 & 1.693 & ${ }^{27} A \ell(n, \alpha)$ & $5.61 E-4$ & $2.351 E-3$ \\
\hline${ }^{6} L i(n, \alpha)$ & 0.376 & 1.578 & ${ }^{48} T i(n, p)$ & $2.21 E-4$ & $9.25 \mathrm{E}-4$ \\
\hline Capture & & & Additions & & \\
\hline $238 \mathrm{U}$ & 0.0583 & 0.245 & $10{ }^{3} R h\left(n, n^{\prime}\right)$ & 0.552 & 2.314 \\
\hline $232 \mathrm{Th}$ & 0.0762 & 0.320 & ${ }^{93} \mathrm{Nb}\left(n, n^{\prime}\right)$ & 0.1248 & 0.523 \\
\hline $197 \mathrm{Au}$ & 0.0656 & 0.2749 & ${ }^{32} S(n, p)$ & 0.0547 & 0.229 \\
\hline${ }^{59} \mathrm{Co}$ & $5.08 E-3$ & 0.0213 & & & \\
\hline${ }^{58} \mathrm{Fe}$ & $1.385 \mathrm{E}-3$ & $5.81 \mathrm{E}-3$ & & & \\
\hline
\end{tabular}


TABLE X-14a(B5). CALCULATED SPECTRAL INDEXES-ENDF/B-V EVALUATED FISSION SPECTRUM

Spectrum: $235 \mathrm{U}$ Thermal Neutron-Induced Fission (ENDF/B-V Nuclear Data File)

Spectral Index: Fu1]-Spectrum Cross Section

(ENDF/B-V Dosimetry File)
Designation: XU5-9-E5

Entry Date: March, 1982

Revised:

$$
\left[S_{\alpha / \beta}\right]_{\text {calc. }} \equiv\left[\bar{\sigma}_{\alpha} / \bar{\sigma}_{\beta}\right]_{\text {calc. }} \text {. }
$$

\begin{tabular}{|c|c|c|c|c|c|}
\hline$\beta$ & $\begin{array}{l}235 U(n, f) \\
;=1.236 b)\end{array}$ & $\begin{array}{c}238 U(n, f) \\
(\bar{\sigma}=0.3052 b)\end{array}$ & & $\begin{array}{l}235 U(n, f) \\
\bar{\sigma}=1.236 b)\end{array}$ & $\begin{array}{l}238 U(n, f) \\
(\bar{\sigma}=0.3052 b)\end{array}$ \\
\hline \multicolumn{3}{|l|}{ Fission } & \multicolumn{3}{|l|}{ Threshold } \\
\hline $239 \mathrm{pu}$ & 1.449 & 5.87 & ${ }^{115} \operatorname{In}\left(n, n^{\prime}\right)$ & 0.1451 & 0.587 \\
\hline $235 U$ & 1.000 & 4.05 & ${ }^{47} \mathrm{Ti}(\mathrm{n}, \mathrm{p})$ & 0.01817 & 0.0736 \\
\hline $233 U$ & 1.543 & 6.25 & ${ }^{58} \mathrm{Ni}(\mathrm{n}, \mathrm{p})$ & 0.0850 & 0.344 \\
\hline $238 U$ & 0.2469 & 1.000 & ${ }^{54} \mathrm{Fe}(\mathrm{n}, \mathrm{p})$ & 0.0656 & 0.2655 \\
\hline $237 \mathrm{~Np}$ & 1.090 & 4.41 & ${ }^{46} T i(n, p)$ & $9.04 E-3$ & 0.0366 \\
\hline \multicolumn{3}{|l|}{ He Prod. } & ${ }^{56} \mathrm{Fe}(\mathrm{n}, \mathrm{p})$ & $8.38 E-4$ & $3.39 E-3$ \\
\hline${ }^{10} B(n, \alpha)$ & 0.397 & 1.607 & ${ }^{63} \mathrm{Cu}(n, \alpha)$ & $4.51 E-4$ & $1.829 E-3$ \\
\hline${ }^{6} \mathrm{Li}(n, \alpha)$ & 0.368 & 1.489 & ${ }^{27} A \ell(n, \alpha)$ & $5.82 E-4$ & $2.357 E-3$ \\
\hline \multicolumn{3}{|l|}{ Capture } & ${ }^{48} T i(n, p)$ & $2.280 E-4$ & $9.23 E-4$ \\
\hline $238 U$ & 0.0568 & 0.2302 & Additions & & \\
\hline${ }^{197} \mathrm{Au}$ & 0.0633 & 0.2565 & $103 \operatorname{Rh}\left(n, n^{\prime}\right)$ & 0.564 & 2.284 \\
\hline${ }^{59} \mathrm{Co}$ & $5.00 E-3$ & 0.02023 & ${ }^{93} \mathrm{Nb}\left(n, n^{\prime}\right)$ & 0.1292 & 0.523 \\
\hline${ }^{58} \mathrm{Fe}$ & $1.367 E-3$ & $5.53 E-3$ & ${ }^{32} S(n, p)$ & 0.0570 & 0.231 \\
\hline
\end{tabular}


TABLE X-14a.1. CALCULATED SPECTRAL INDEX RATI0: NBS EVALUATION VS. ENDF/B-V EVALUATED FISSION SPECTRUM

Spectrum: $235 \mathrm{U}$ Thermal Neutron-Induced Fission (NBS Eval./ENDF/B-V)

Spectral Index: Fu11-Spectrum Cross Section

(ENDF/B-V Dosimetry File)
Designation: $\frac{X U 5-5-N 1}{X U 5-9-E 5}$

Entry Date: March, 1982

$$
\left[\mathrm{S}_{\alpha / \beta}\right]_{\chi_{N B S}} /\left[\mathrm{S}_{\alpha / \beta}\right]_{\chi_{B V}}
$$

\begin{tabular}{|c|c|c|c|c|c|}
\hline$\alpha \beta$ & ${ }^{235} U(n, f)$ & $238 u(n, f)$ & $\alpha \beta$ & $235 U(n, f)$ & $238 U(n, f)$ \\
\hline Fission & & & Threshold & & \\
\hline $239 \mathrm{pu}$ & 0.997 & 1.032 & ${ }^{115} \operatorname{In}\left(n, n^{\prime}\right)$ & 0.9671 & 1.001 \\
\hline $235 U$ & 1.000 & 1.035 & ${ }^{47} \mathrm{Ti}(n, p)$ & 0.962 & 0.996 \\
\hline $233 U$ & 1.003 & 1.038 & ${ }^{58} \mathrm{Ni}(n, p)$ & 0.960 & 0.994 \\
\hline $238 U$ & 0.966 & 1.000 & ${ }^{54} \mathrm{Fe}(n, p)$ & 0.960 & 0.994 \\
\hline $237 \mathrm{~Np}$ & 0.982 & 1.018 & ${ }^{46} \mathrm{Ti}(n, p)$ & 0.971 & 1.003 \\
\hline He Prod. & & & ${ }^{56} \mathrm{Fe}(n, p)$ & 0.971 & 1.006 \\
\hline${ }^{10} B(n, \alpha)$ & 1.018 & 1.053 & ${ }^{63} \mathrm{Cu}(n, \alpha)$ & 0.968 & 1.002 \\
\hline${ }^{6} \operatorname{Li}(n, \alpha)$ & 1.022 & 1.060 & ${ }^{27} \mathrm{Al}(n, \alpha)$ & 0.965 & 0.997 \\
\hline Capture & & & ${ }^{48} \mathrm{Ti}(n, p)$ & 0.970 & 1.002 \\
\hline $238 U$ & 1.026 & 1.064 & Additions & & \\
\hline${ }^{197} \mathrm{Au}$ & 1.036 & 1.072 & ${ }^{103} \operatorname{Rh}\left(n, n^{\prime}\right)$ & 0.979 & 1.013 \\
\hline${ }^{59} \mathrm{Co}$ & 1.018 & 1.053 & ${ }^{93} \mathrm{Nb}\left(n, n^{\prime}\right)$ & 0.966 & 1.000 \\
\hline${ }^{58} \mathrm{Fe}$ & 1.013 & 1.051 & ${ }^{32} S(n, p)$ & 0.960 & 0.991 \\
\hline
\end{tabular}


TABLE $X-15(B 5)$. CALCULATED SPECTRAL INDEXES--TRUNCATED

Spectrum: $235 \mathrm{U}$ Thermal-Neutron-Induced Fission (NBS evaluation, $[\mathrm{Gr} 75 \mathrm{~b}],[\mathrm{Gr} 75 \mathrm{c}]$ )

Spectral Index: Truncated Cross Sections from Table $X-13(B 5)$, Column 3 (ENDF/B-V Dosimetry File)
Designation: $\quad X U 5-5-N 1$

Entry Date: June, 1978

Revised: March, 1982

$$
\left[S_{\alpha / \beta}\right]_{\text {calc. }} \equiv\left[\bar{\sigma}_{\alpha}\left(>E_{95}\right) / \bar{\sigma}_{\beta}\left(>E_{95}\right)\right]_{\text {calc. }}
$$

\begin{tabular}{|c|c|c|c|}
\hline$\alpha \beta$ & $\begin{array}{l}238 U(n, f) \\
95)=0.532 b\end{array}$ & & $\begin{array}{l}8 U(n, f) \\
5)=0.532 b\end{array}$ \\
\hline \multicolumn{2}{|l|}{ Fission } & \multicolumn{2}{|l|}{ Threshold } \\
\hline${ }^{239} \mathrm{Pu}$ & 3.38 & ${ }^{115} \operatorname{In}\left(n, n^{\prime}\right)$ & 0.496 \\
\hline $235 U$ & 2.295 & ${ }^{47} \mathrm{Ti}(n, p)$ & 0.0915 \\
\hline $233 \mathrm{U}$ & 3.56 & ${ }^{32} S(n, p)$ & 0.372 \\
\hline $238 U$ & 1.000 & ${ }^{58} \mathrm{Ni}(n, p)$ & 0.497 \\
\hline $23^{7} \mathrm{~Np}$ & 2.97 & ${ }^{54} \mathrm{Fe}(n, p)$ & 0.439 \\
\hline \multirow[t]{2}{*}{$240 \mathrm{Pu}$} & 2.98 & ${ }^{46} T i(n, p)$ & 0.1515 \\
\hline & & ${ }^{56} \mathrm{Fe}(n, p)$ & 0.0461 \\
\hline Additions & & ${ }^{63} \mathrm{Cu}(n, \alpha)$ & 0.01466 \\
\hline${ }^{103} \operatorname{Rh}\left(n, n^{\prime}\right)$ & 1.570 & ${ }^{48} \mathrm{Ti}(n, p)$ & 0.01731 \\
\hline $93 \mathrm{Nb}\left(n, n^{\prime}\right)$ & 0.406 & ${ }^{27} \mathrm{Al}(n, \alpha)$ & 0.0665 \\
\hline
\end{tabular}


TABLE $X-16$. OBSERVED INTEGRAL CROSS SECTIONS

Spectrum: $235 \mathrm{U}$ Thermal-Neutron-Induced Fission Designation: XU5

Entry Date: March, 1982

Revised: Jul y, 1985

\begin{tabular}{|c|c|c|c|c|}
\hline Reaction & $\begin{array}{l}\text { Median Response } \\
\text { Energy (MeV) }\end{array}$ & $\begin{array}{r}\text { Cros } \\
\text { Secti } \\
\left(\times 10^{27}\right.\end{array}$ & $\begin{array}{l}\text { ss } \\
\text { ion } \\
\mathrm{cm}^{2} \text { ) }\end{array}$ & $\begin{array}{l}\text { Measurement } \\
\text { Reference }\end{array}$ \\
\hline & Threshold Reactions & & & \\
\hline${ }^{238} U(n, f)^{\dagger}$ & 2.7 & 312 & $\pm 2.3 \%$ & [Gi84a], [Le57a] \\
\hline $23{ }^{7} \mathrm{~Np}(\mathrm{n}, \mathrm{f})^{\dagger}$ & 1.96 & 1359 & $\pm 2.1 \%$ & {$[\mathrm{Gi} 84 \mathrm{a}]$} \\
\hline $240 \mathrm{Pu}(\mathrm{n}, \mathrm{f})^{\dagger}$ & 1.97 & 1332 & $\pm 2.1 \%$ & [Gi84a] \\
\hline${ }^{232} \operatorname{Th}(n, f)^{\dagger}$ & 2.9 & 82.9 & $\pm 3.1 \%$ & {$[$ Gi 84a] } \\
\hline${ }^{93} \mathrm{Nb}\left(n, n^{\prime}\right)$ & 2.4 & 164 & $\pm 9 \%$ & {$[\mathrm{Al} 82 \mathrm{a}],[\mathrm{Ko80a}]$} \\
\hline${ }^{115} \operatorname{In}\left(n, n^{\prime}\right)$ & 2.6 & 190 & $\pm 2.1 \%$ & $\begin{array}{c}{[\mathrm{Gi} 84 \mathrm{a}],[\mathrm{Fa} 75 \mathrm{~b}],} \\
{[\mathrm{Ki} 78 \mathrm{a}]}\end{array}$ \\
\hline${ }^{47} \mathrm{Ti}(\mathrm{n}, \mathrm{p})$ & 3.8 & 18.0 & $\pm 3.5 \%$ & {$[\mathrm{Ma} 84 \mathrm{~b}],[\mathrm{Ki} 78 \mathrm{a}]$} \\
\hline${ }^{58} \mathrm{Ni}(\mathrm{n}, \mathrm{p})$ & 4.1 & 111 & $\pm 2.4 \%$ & $\begin{array}{l}{[\mathrm{Gi} 84 \mathrm{a}],[\mathrm{La} 82 \mathrm{a}],} \\
{[\mathrm{Fa75a}],[\mathrm{Fa} 74 \mathrm{a}]}\end{array}$ \\
\hline${ }^{54} \mathrm{Fe}\left(\mathrm{n}, \mathrm{p}^{\prime}\right)$ & 4.2 & 81.7 & $\pm 2.7 \%$ & $\begin{array}{c}{[\text { Ma84b], }[\text { La82a }],} \\
{[\mathrm{F} 777 \mathrm{a}]}\end{array}$ \\
\hline${ }^{46} T i(n, p)$ & 5.7 & 11.8 & $\pm 3.5 \%$ & {$[\mathrm{Ma} 84 \mathrm{~b}]$} \\
\hline${ }^{63} \mathrm{Cu}(n, \alpha)$ & 7.3 & 0.60 & $\pm 7 \%$ & {$[\mathrm{Fa} 75 \mathrm{a}],[\mathrm{Ki} 78 \mathrm{a}]$} \\
\hline${ }^{48} \mathrm{Ti}(\mathrm{N}, \mathrm{p})$ & 8.1 & 0.307 & $\pm 3.5 \%$ & {$[\mathrm{Ma} 84 \mathrm{~b}],[\mathrm{Ki78a}]$} \\
\hline${ }^{27} A l(n, \alpha)$ & 8.4 & 0.720 & $\pm 3.5 \%$ & {$[\mathrm{Ma} 84 \mathrm{~b}],[\mathrm{Fa} 75 \mathrm{a}]$} \\
\hline \multicolumn{5}{|c|}{ Non-Threshold Reactions } \\
\hline${ }^{239} \mathrm{Pu}(\mathrm{n}, \mathrm{f})^{\dagger}$ & 1.65 & {$[1818$} & $\pm 1.9 \%]$ & {$[\mathrm{Gi} 84 \mathrm{a}],[\mathrm{Mc} 84 \mathrm{a}]$} \\
\hline${ }^{235} U(n, f)^{\dagger}$ & 1.57 & 1200 & $\pm 1.9 \%$ & {$[\mathrm{Gi} 84 \mathrm{a}]$} \\
\hline${ }^{23} 3 U(n, f)^{\dagger}$ & 1.51 & 1949 & $\pm 3.1 \%$ & {$[\mathrm{Gi} 84 \mathrm{a}]$} \\
\hline${ }^{10} \mathrm{~B}(\mathrm{n}, \mathrm{He})$ & 1.16 & 540 & $\pm 4.0 \%$ & {$[\mathrm{Gr} 85 \mathrm{a}],[0182 \mathrm{a}]$} \\
\hline${ }^{6} \mathrm{Li}(\mathrm{n}, \mathrm{He})$ & 1.25 & 455 & $\pm 4.0 \%$ & {$[\mathrm{Gr} 85 \mathrm{a}],[0182 \mathrm{a}]$} \\
\hline
\end{tabular}

${ }^{\dagger}$ Ratio measurements with double fission chambers in the SCK/CEN Cavity Fission Source [Gi84a]. The flux monitor was $239 \mathrm{Pu}(\mathrm{n}, \mathrm{f})$ for which the ${ }^{252} \mathrm{Cf}$ fission spectrum observed value is $(1824 \pm 1.9 \%) \mathrm{mb}--$ see Part IA. The $239 \mathrm{pu}$ fission cross section ratio between ${ }^{252} \mathrm{Cf}$ and $235 \mathrm{U}$ fission spectra is 1.003 based on spectrum eval uation (Section 4.b.3). A conservative upper bound for the error in this ratio is $\pm 0.5 \%$. 
TABLE $X-17(B 5)$. CALCULATED-TO-OBSERVED RATIOS OF CROSS SECTIONS

Spectrum: $235 \mathrm{U}$ Thermal-Neutron-Induced Fission

(NBS-Eval uated, [Gr75b], [Gr75c])
Designation: $\quad X U 5-5-N 1$

Entry Date: March, 1982

Revised: July, 1985

Calcul ated-to-observed

NBS-eval. (b) ENDF/B-V

fiss. spec. fiss. spec.

Cross Section: ENDF/BV Dosimetry File

Calcul ated

REACTION

(Table $X-16)$

fiss. spec.

fiss.

\begin{tabular}{l|l} 
(Table $X-16)$ \\
\hline
\end{tabular}

Fission Reactions ${ }^{\dagger}$

239Pu $(n, f) \quad[1818 \pm 1.9 \%]$

$235 U(n, f) \quad 1200$

$233 \cup(n, f)$

1949

$\pm 1.9 \%$

$\pm 3.1 \%$

$238 U(n, f)$

312

$\pm 2.3 \%$

$237 \mathrm{~Np}(n, f)$

1359

$232 \operatorname{Th}(n, f)$

$82.9 \pm 3.1 \%$

$240 \mathrm{Pu}(\mathrm{n}, \mathrm{f})$

1332

$\pm 2.1 \%$

Hel i um Production

\begin{tabular}{c|ll|lll|ll}
$10_{\mathrm{B}}(\mathrm{n}, \mathrm{He})$ & 540 & $\pm 4.0 \%$ & 499 & $\pm 2.3 \%$ & 491 & $0.923 \pm 4.6 \%$ & 0.909 \\
${ }^{6} \mathrm{Li}(\mathrm{n}, \mathrm{He})$ & 455 & $\pm 4.0 \%$ & 465 & $\pm 2.0 \%$ & 455 & $1.021 \pm 4.5 \%$ & 1.000
\end{tabular}

Threshold Reactions

\begin{tabular}{|c|c|c|c|c|c|c|}
\hline $9{ }^{3} \mathrm{Nb}\left(n, n^{\prime}\right)$ & 164 & $\pm 9 \%$ & $\pm 1.4 \%$ & 159.7 & $0.94 \pm 9.1 \%$ & 0.97 \\
\hline${ }^{115} \operatorname{In}\left(n, n^{\prime}\right)$ & 190 & $\pm 2.1 \%$ & $\pm 1.4 \%$ & 179.3 & $0.911 \pm 2.5 \%$ & 0.944 \\
\hline${ }^{47} \mathrm{Ti}(n, p)$ & 18.0 & $\pm 3.5 \%$ & $\pm 2.2 \%$ & 22.5 & $1.200 \pm 4.1 \%$ & 1.250 \\
\hline${ }^{58} \mathrm{Ni}(n, p)$ & 111. & $\pm 2.4 \%$ & $\pm 2.6 \%$ & 105.0 & $0.909 \pm 3.5 \%$ & 0.946 \\
\hline${ }^{54} \mathrm{Fe}(n, p)$ & 81.7 & $\pm 2.7 \%$ & $\pm 2.7 \%$ & 81.0 & $0.952 \pm 3.8 \%$ & 0.991 \\
\hline${ }^{46} \mathrm{Ti}(n, p)$ & 11.8 & $\pm 3.5 \%$ & $\pm 3.8 \%$ & 11.2 & $0.915 \pm 5.2 \%$ & 0.949 \\
\hline${ }^{63} \mathrm{Cu}(n, \alpha)$ & $0.60 \pm$ & $\pm 7 \%$ & $0.540 \pm 3.4 \%$ & 0.558 & $\pm 7.8 \%$ & 0.93 \\
\hline${ }^{48} \mathrm{Ti}(n, p)$ & $0.307=$ & $\pm 3.5 \%$ & $0.273 \pm 3.3 \%$ & 0.282 & $0.889 \pm 4.8 \%$ & 0.958 \\
\hline${ }^{27} \mathrm{Al}(n, \alpha)$ & $0.720=$ & $\pm 3.5 \%$ & $0.693 \pm 3.6 \%$ & 0.719 & $0.963 \pm 5.0 \%$ & 0.999 \\
\hline
\end{tabular}

(a) Errors correspond to $235 \mathrm{U}$ fission spectrum uncertainties ( 10 ) given in Table $\mathrm{X}-5$ and propagated according to the second term of Eq. $(X-7)$, Section 5.a.3., Part lA.

(b) Errors are quadrature sum of errors in columns 2 and 3.

${ }^{\dagger}$ See footnote in Table $x-16$. 
TABLE X-18. OBSERVED SPECTRAL INDEXES

Spectrum: $235 \mathrm{U}$ Thermal-Neutron-Induced Fission

Spectrum: 235 Thermal -Neutron-Induced Fission

$$
\left[\mathrm{s}_{\alpha / \mathrm{U} 8}\right]_{\mathrm{obs}}=\left[\bar{\sigma}_{\alpha}{ } \bar{\sigma}_{f}(\mathrm{U} 238)\right]_{\mathrm{obs}}
$$

Designation: XU5

Entry Date: May, 1978

Revised: July, 1985

\begin{tabular}{|c|c|c|c|}
\hline Reaction (a) & $\begin{array}{l}\text { Reaction (b) } \\
\text { Non-overl ap } \\
\text { Interval (MeV) }\end{array}$ & $\begin{array}{l}\text { Spectral (c) } \\
\text { Index }\end{array}$ & $\begin{array}{l}\text { Measurement } \\
\text { Reference }\end{array}$ \\
\hline \multicolumn{4}{|l|}{ Threshold Reactions } \\
\hline$\overline{237} \mathrm{~Np}(n, f) / U 8^{\dagger}$ & $0.67-1.5$ & $\pm 1.7 \%$ & [Gi84a] \\
\hline${ }^{240} \mathrm{Pu}(\mathrm{n}, \mathrm{f}) / \mathrm{U} 8^{\dagger}$ & $0.67-1.5$ & $\pm 1.7 \%$ & [Gi84a] \\
\hline${ }^{93} \mathrm{Nb}\left(n, n^{\prime}\right) \cup 8$ & $0.99-1.5$ & $0.526 \pm 9 \%$ & [A1 82a] \\
\hline $115 \operatorname{In}\left(n, n^{\prime}\right) / U 8$ & $1.2-1.5$ & $0.609 \pm 2.0 \%$ & {$[\mathrm{Gi} 84 \mathrm{a}],[\mathrm{Fa} 75 \mathrm{a}]$} \\
\hline${ }^{47} \mathrm{Ti}(\mathrm{n}, \mathrm{p}) / \mathrm{U} 8$ & $1.9-1.5$ & $0.0577 \pm 4.2 \%$ & {$[\mathrm{Ma} 84 \mathrm{~b}],[\mathrm{Ki} 78 \mathrm{a}]$} \\
\hline${ }^{58} \mathrm{Ni}(\mathrm{n}, \mathrm{p}) / \mathrm{U} 8$ & $2.1-1.5$ & $0.357 \pm 2.0 \%$ & $\begin{array}{c}{[\mathrm{Gi} 84 \mathrm{a}],[\mathrm{La} 82 \mathrm{a}],} \\
{[\mathrm{Fa75a}]}\end{array}$ \\
\hline${ }^{54} \mathrm{Fe}(\mathrm{n}, \mathrm{p}) / \mathrm{U} 8$ & $2.5-1.5$ & $0.262 \pm 3.5 \%$ & {$[\mathrm{Ma} 84 \mathrm{~b}],[\mathrm{La} 82 \mathrm{a}]$} \\
\hline${ }^{46} \mathrm{Ti}(\mathrm{n}, \mathrm{p}) / \mathrm{U} 8$ & $3.8-1.5$ & $0.0378 \pm 3.0 \%$ & {$[\mathrm{Ma} 84 \mathrm{~b}]$} \\
\hline${ }^{63} \mathrm{Cu}(\mathrm{n}, \alpha) / \mathrm{U} 8$ & $4.7-1.5$ & $1.92 \mathrm{E}-3 \pm 10 \%$ & {$[\mathrm{Fa75a}],[\mathrm{Ki78a}]$} \\
\hline${ }^{48} \mathrm{Ti}(\mathrm{n}, \mathrm{p}) / \mathrm{U} 8$ & $5.9-1.5$ & $0.984 \mathrm{E}-3 \pm 4.2 \%$ & {$[\mathrm{Ma} 84 \mathrm{~b}],[\mathrm{Ki78a}]$} \\
\hline${ }^{27} \mathrm{Al}(\mathrm{n}, \alpha) / \mathrm{U} 8$ & $6.5-1.5$ & $2.31 E-3 \pm 4.2 \%$ & {$[\mathrm{Ma} 84 \mathrm{~b}],[\mathrm{Fa} 75 \mathrm{a}]$} \\
\hline \multicolumn{4}{|l|}{ Non-Threshold Reactions } \\
\hline${ }^{239} \mathrm{Pu}(\mathrm{n}, \mathrm{f}) / \mathrm{U} 8^{\dagger}$ & $0.26-1.5$ & $\pm 1.7 \%$ & [Gi84a] \\
\hline${ }^{235} u(n, f) / U 8^{\dagger}$ & $0.20-1.5$ & $\pm 1.7 \%$ & [Gi84a] \\
\hline${ }^{23} 3 u(n, f) / U 8^{\dagger}$ & $0.21-1.5$ & $\pm 3.0 \%$ & [Gi84a] \\
\hline${ }^{10} \mathrm{~B}(\mathrm{n}, \mathrm{He}) / \mathrm{U} 8$ & $0.07-1.5$ & $\pm 4.5 \%$ & {$[\mathrm{Gr} 85 \mathrm{a}],[0182 \mathrm{a}]$} \\
\hline${ }^{6} \mathrm{Li}(\mathrm{n}, \mathrm{He}) / \mathrm{U} 8$ & $0.17-1.5$ & $\pm 4.5 \%$ & {$[\operatorname{Gr} 85 \mathrm{a}],[0182 \mathrm{a}]$} \\
\hline
\end{tabular}

(a) All spectral indexes are taken relative to the $238 U(n, f)$ reaction 1 abel ed "U8".

(b) Non-overlapping energy response interval between the $95 \%$ response energy boundary for each detector. See Table X-13(B5) and footnote (b).

(c) Fission detector indexes are from simul taneous measurement of fission rates from both isotopes and hence show smaller uncertainties because of reduced neutron fluence errors. Other spectral indexes are formed from independent cross section measurements 1 isted in Table $X-16$.

${ }^{\dagger}$ Ratio measurements obtained with NBS double fission chamber at the SCK/CEN cavity fission source. 
TABLE $X-19(B 5)$. CALCIJLATED-TO-OBSERVED RATIOS OF SPECTRAL INDEXES - NBS EVALUATION

Spectrum: $235 \mathrm{U}$ Thermal-Neutron-Induced Fission

(NBS evaluation, [Gr75b], [Gr75c])

Spectral Index: Calculated: Table $x-14$ (B5)

Observed: Table $X-18$
Designation: $\quad X \cup 5-5 N 1$

Entry Date: May, 1978

Revised: July, 1985

$C_{\alpha / U 8}=\left[S_{\alpha / U 8}\right]_{c a l c} /\left[S_{\alpha / U 8}\right]_{0 b s}$. or $C_{U 8 / \alpha}$

\begin{tabular}{|c|c|c|c|c|c|}
\hline Reaction(a) & $\begin{array}{l}\text { Reaction (b) } \\
\text { Non-overl ap } \\
\text { Interval (MeV) }\end{array}$ & $\begin{array}{c}\text { Calcul ated- } \\
\text { To-Observed } \\
\text { Values }\end{array}$ & $\begin{array}{l}\text { Observed } \\
\text { Value }\end{array}$ & $\begin{array}{l}\text { R R O R S }(1 \sigma) \\
\text { Calculated }(c) \\
\text { Value }\end{array}$ & Total \\
\hline \multicolumn{6}{|c|}{ Threshold Reactions } \\
\hline $\mathrm{U} 8 /{ }^{237} \mathrm{~Np}(\mathrm{n}, \mathrm{f})$ & $0.67-1.5$ & 0.971 & $\pm 1.7 \%$ & $\pm 1.0 \%$ & $\pm 2.0 \%$ \\
\hline$U 8 /{ }^{9} 3 \mathrm{Nb}\left(n, n^{\prime}\right)$ & $0.99-1.5$ & 1.01 & $\pm 9 \%$ & - & - \\
\hline U8/ $115 \operatorname{In}\left(n, n^{\prime}\right)$ & $1.2-1.5$ & 1.036 & $\pm 2.0 \%$ & $\pm 0.3 \%$ & $\pm 2.0 \%$ \\
\hline${ }^{47} \mathrm{Ti}(\mathrm{n}, \mathrm{p}) / \mathrm{U8}$ & $1.5-1.9$ & 1.27 & $\pm 4.2 \%$ & $\pm 1.1 \%$ & $\pm 4.3 \%$ \\
\hline${ }^{58} \mathrm{Ni}(\mathrm{n}, \mathrm{p}) / \mathrm{U} 8$ & $1.5-2.1$ & 0.958 & $\pm 2.0 \%$ & $\pm 1.7 \%$ & $\pm 2.6 \%$ \\
\hline${ }^{54} \mathrm{Fe}(n, p) / \mathrm{U} 8$ & $1.5-2.5$ & 1.008 & $\pm 3.5 \%$ & $\pm 1.9 \%$ & $\pm 4.0 \%$ \\
\hline${ }^{46} \mathrm{Ti}(n, p) / \mathrm{U} 8$ & $1.5-3.8$ & 0.971 & $\pm 3.0 \%$ & $\pm 3.1 \%$ & $\pm 4.3 \%$ \\
\hline${ }^{63} \mathrm{Cu}(n, \alpha) / 48$ & $1.5-4.7$ & 0.954 & $\pm 10 \%$ & $\pm 2.8 \%$ & $\pm 10.4 \%$ \\
\hline${ }^{48} \mathrm{Ti}(\mathrm{n}, \mathrm{p}) / \mathrm{U} 8$ & $1.5-5.9$ & 0.940 & $\pm 4.2 \%$ & $\pm 2.8 \%$ & $\pm 5.0 \%$ \\
\hline${ }^{27} \mathrm{Al}(\mathrm{n}, \alpha) / \mathrm{U} 8$ & $1.5-6.5$ & 1.018 & $\pm 4.2 \%$ & $\pm 3.2 \%$ & $\pm 5.3 \%$ \\
\hline \multicolumn{6}{|c|}{ Non-Threshold Reactions } \\
\hline$u 8 / 239 p u(n, f)$ & $\begin{array}{l}0.3-1.5 \text { low } \\
5.2-6.7 \text { high }\end{array}$ & 0.962 & $\pm 1.7 \%$ & $\pm 1.4 \%$ & $\pm 2.2 \%$ \\
\hline$U 8 / 235 U(n, f)$ & $\begin{array}{l}0.2-1.5 \frac{10 \mathrm{ow}}{\mathrm{high}} \\
5.2-6.7\end{array}$ & 0.919 & $\pm 1.7 \%$ & $\pm 1.7 \%$ & $\pm 2.4 \%$ \\
\hline $\mathrm{U} 8 /{ }^{1} \mathrm{O}_{\mathrm{B}}(\mathrm{n}, \mathrm{He})$ & $\begin{array}{l}0.066-1.5 \text { low } \\
5.2-6.7 \text { high }\end{array}$ & 1.022 & $\pm 4.5 \%$ & $\pm 3.4 \%$ & $\pm 5.6 \%$ \\
\hline $\mathrm{U} 8 /{ }^{6} \mathrm{Li}(\mathrm{n}, \mathrm{He})$ & $\begin{array}{l}0.17-1.5 \frac{10 w}{10} \\
5.7-6.7 \text { high }\end{array}$ & 0.925 & $\pm 4.5 \%$ & $\pm 3.1 \%$ & $\pm 5.5 \%$ \\
\hline
\end{tabular}

(a) Spectral indexes are taken with respect to the $238 \mathrm{U}(\mathrm{n}, \mathrm{f})$ reaction 1 abeled "U8" with the lower energy response detector in the denominator. The calculated-to-observed ratio for $\bar{\sigma}_{f}($ U238) is 0.945 (NBS-eval.) from Table $X-17(B 5)$.

(b) Non-overlapping energy response interval between the $95 \%$ response energy boundary for each detector. For non-threshold reactions, two nonoverlapping energy intervals are listed: The $95 \%$ exclusion interval is labeled "low" and the 5\% exclusion interval "high". See Table X-13.

(c) Errors in the calculated spectral indexes are for $235 y$ fission spectrum uncertainties only as given in Table $X-5$ and propagated according to the second term of Eq. $(X-8)$, Part IA, Sect. 5.a.3. The error in $\bar{\sigma}_{f}($ U238) calculated is $\pm 1.5 \%$ from Table $X-17(B 5)$. 


\section{NBS CAVITY FISSION SOURCE}

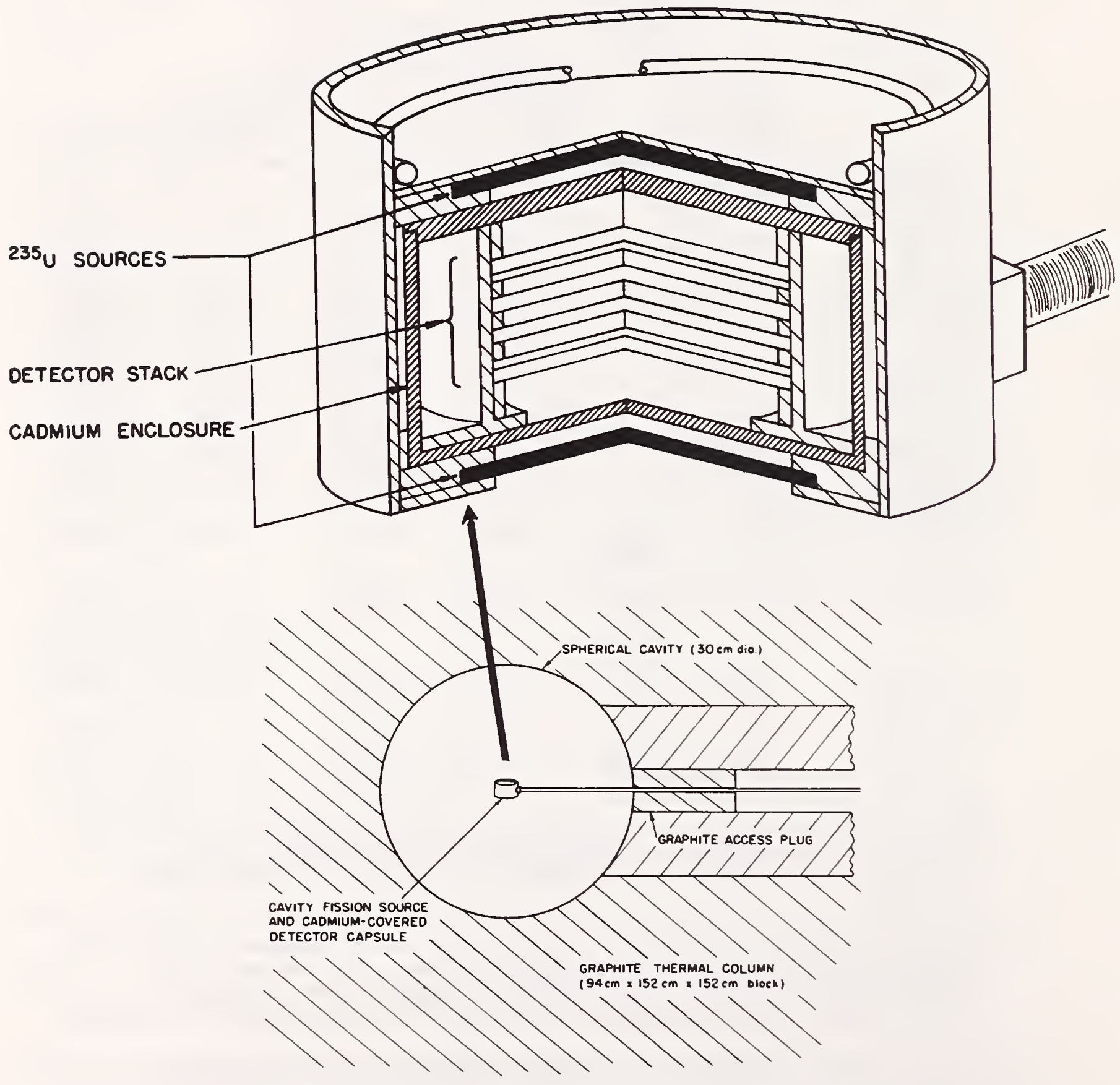

Figure $\mathrm{X}-3$. NBS Cavity Fission Source and detector assembly 

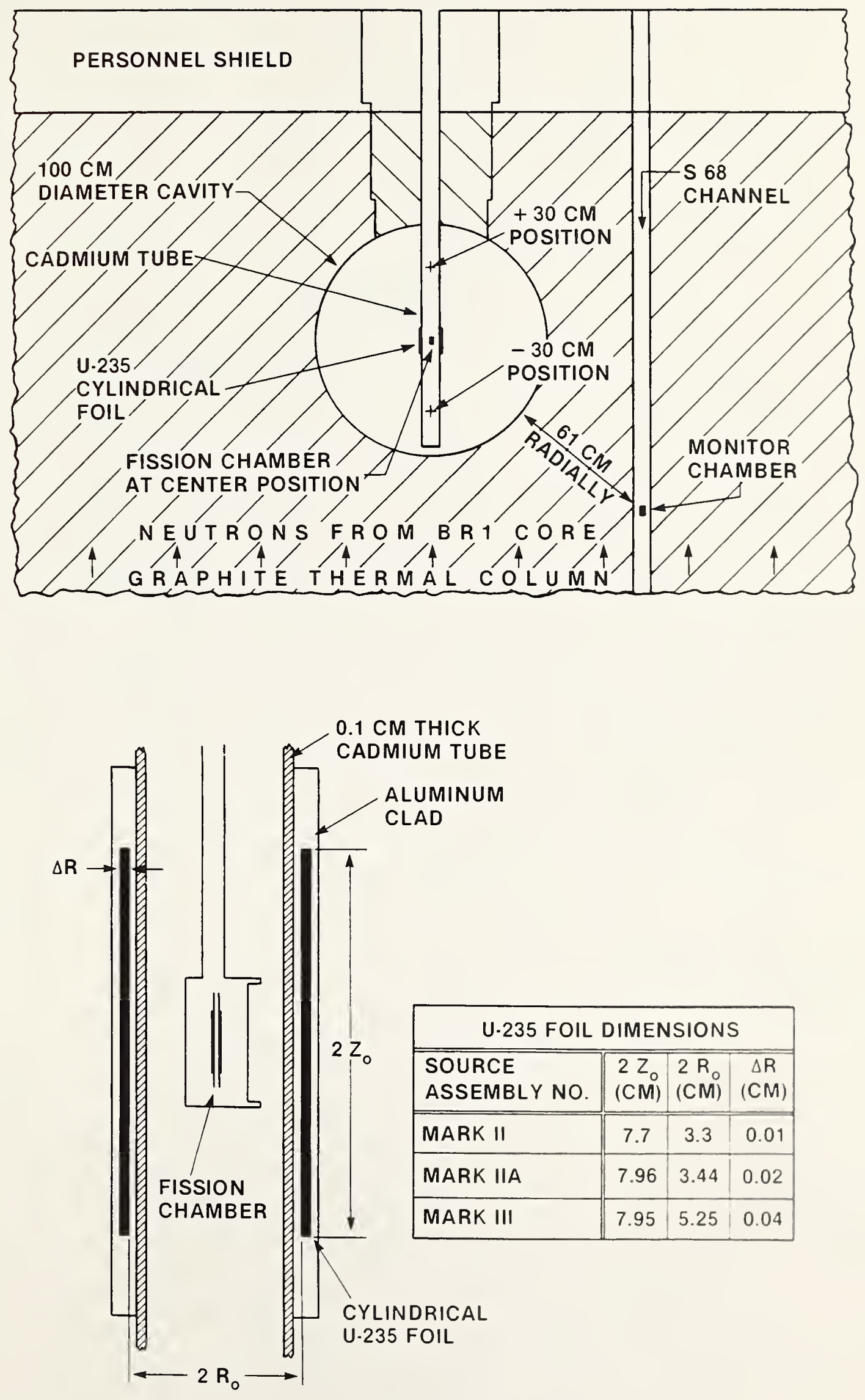

Figure X-4. Cavity Fission Source Irradiation Facility at SCK/CEN Belgium 



Part IIA: Intermediate-Energy Standard Neutron Fields (ISNF) With Thick ${ }^{10} \mathrm{~B}$ Shell 
1. IDENTIFICATIONS. . . . . . . . . . . . 75

1.a. Title, Classification

1.b. Designation

1.c. Entry Dates and Revisions

1.d. Generic Description

1.e. Facility Location

1.f. Contacts for Information

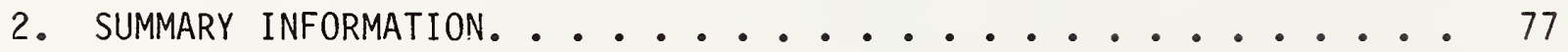

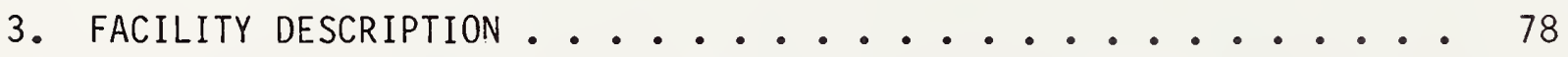

3.a. Configuration and Characteristics

3.b. Irradiation Procedures

(experimental arrangements and fluence monitoring)

3.c. Specification for Neutron Transport Calculation

(materials and geometry)

4. NEUTRON FIELD CHARACTERIZATION .............

4.a. Neutron Fluence

4.b. Neutron Spectrum

(intensity, fluence transfer, gradients)

4.b.1. Calculation

(sensitivity study)

4.b.2. Measurement

4.b.3. Evaluated Spectrum

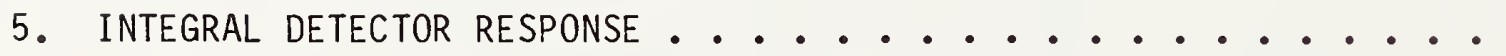

5.a. Calculated Reaction Probabilities

5.a.1. Spectrum Response and Error Propagation Tables

(cross sections, spectrum fraction, response range,

5.a.2. Spectral Indexes

propagation of spectrum errors)

5.a.3. Spectral Index Errors

5.b. Measured Reaction Probabilities

5.c. Measured Cross Sections and Calculated-to-Observed Ratios

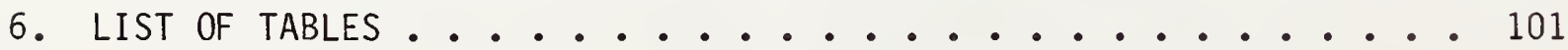

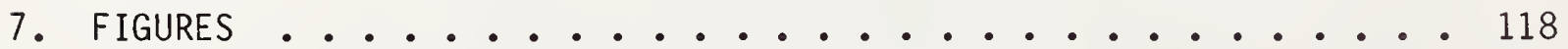




\section{IDENTIFICATIONS}

1.a. NEUTRON FIELD: INTERMEDIATE-ENERGY STANDARD NEUTRON FIELD (ISNF) WITH THICK $10 \mathrm{~B}$ SHELL

\section{CLASSIFICATION: STANDARD NEUTRON FIELD}

1.b. DESIGNATION: $\operatorname{ISNF}(5)-1-L 1$

Discrete ordinates transport calculations (LASL 150-group

ONEDANT) with ENDF/B-V boron and carbon cross sections and with the fission source spectrum taken as the average of the ENDF/B-V and NBS evaluations. References: $[$ Br79a], [Ei77a], [La80a], [So79a]

\section{C. ENTRY DATE: JuTy, 1983 \\ REVISIONS: JuTy, 1985}

1.d. GENERIC DESCRIPTION:

The Intermediate-Energy Neutron Field (ISNF) is an irradiation facility designed to produce a strong component of neutrons in the energy range of interest for fast-reactor and related technologies. Arranged in spherical geometry, driven by fission neutrons, and employing two materials with uniquely well-understood nuclear properties for neutron transport, the facility provides a constant flux of neutrons whose spectrum may be accurately calculated. General properties of the neutron field at the center of ISNF are as follows:

Neutron fluence rate

Irradiation space
$1.5 \times 10^{9} \mathrm{n} / \mathrm{cm}^{2} \mathrm{~s}$

$5 \mathrm{~cm}$ dia. sphere 
Spectrum:

Energy range

Median energy
$98 \%$ of fluence between $1.2 \mathrm{keV}$ and $5.7 \mathrm{MeV}$

$0.58 \mathrm{MeV}$

Median response energy for reaction with $1 / v$ cross section $25 \mathrm{keV}$

Neutron transport in ISNF is determined almost entirely by fission neutron scattering and diffusion in carbon, and by neutron absorption in $10 \mathrm{~B}$.

The resulting neutron spectrum, establ ished on the basis of neutron transport calculations in 1-D geometry, is limited in accuracy only by the uncertainty in the driving spectrum and the input cross section data. Such calculations, employing both discrete-ordinates and Monte-Carlo methods, have been carried out by NBS [Ei77a], Los Al amos [La80a], [So79a], and 0ak Ridge [Wa81a]. The simplicity of a 1-D calculation permits detailed investigation of spectrum sensitivity to uncertainties in physical and nuclear parameters of the system (mainly material densities and cross sections of carbon and $10 \mathrm{~B}$ ), and spectrum perturbations due to extraneous materials required for fabrication.

The neutron fluence in ISNF has been establ ished to an accuracy of $\pm 2.1 \%$ by means of neutron fluence transfer from the ${ }^{252} \mathrm{Cf}$ fission neutron standard field [Gr75c], [Gr77b], [La84a]. Fission cross sections for most fissionable isotopes of interest for nuclear technology have been measured in the facility with fission ionization chambers; other measurements include capture cross sections in gold and $238 \mathrm{U}$, and He-production in $10_{\mathrm{B}}$ and $6 \mathrm{Li}$. 
1.e. FACILITY LOCATION:

National Bureau of Standards

Center for Radiation Research

Gaithersburg, MD 20899

Contact: Dave Gilliam or James Grundl

Phone: 301-921-2767

1.f. CONTACT FOR INFORMATION:

Charles Eisenhauer or James Grundl

National Bureau of Standards

Center for Radiation Research

Gaithersburg, MD 20899

Phone: 301-921-2767

2. SUMMARY INFORMATION

2.a. AVAILABLE NEUTRON EXPOSURES

- fluence rate

- nominal maximum fluence

- accuracy of free-field fluence ( $1 \sigma)$

- irradiation space
$1.5 \times 10^{9} \mathrm{n} / \mathrm{cm}^{2} \mathrm{~s}$

$1 \times 10^{14} \mathrm{n} / \mathrm{cm}^{2}$

$\pm 2.1 \%$

$5 \mathrm{~cm}$ dia. sphere

2.b. DETECTOR RESPONSE RANGE AND UNCERTAINTY

$$
235 U(n, f) \quad 237 \mathrm{~Np}(n, f) \quad \underline{A u(n, \gamma)}
$$

Energy response range (Table $1-5)$ :

$$
\begin{array}{rlll}
E_{50} \text { (median energy): } & 0.26 \mathrm{MeV} & 1.54 \mathrm{MeV} & 6.5 \mathrm{keV} \\
E_{95}: & 0.90 \mathrm{keV} & 0.53 \mathrm{MeV} & 0.33 \mathrm{keV} \\
E_{05}: & 3.1 \mathrm{MeV} & 4.5 \mathrm{MeV} & 0.97 \mathrm{MeV}
\end{array}
$$

Cross section error associated with spectrum uncertainties (Table I-8):

$$
\pm 0.6 \% \quad \pm 1.2 \% \quad \pm 2.0 \%
$$




\section{FACILITY DESCRIPTION}

The Intermediate-Energy Standard Neutron Field (ISNF) operates in the graphite thermal column of the Research Reactor at the National Bureau of Standards in Gaithersburg, Maryland. As an irradiation facility it is in general use for neutron cross section measurements and detector calibrations. As a primary neutron field standard its physical components and geometric configuration have been extensively investigated and it is these features of the system which are summarized in this section.

3.a. CONFIGURATION AND CHARACTERISTICS [Ei77a], [Gr77c], [Mc82a], [Wa81a] With the reactor at full power, the ISNF facility is assembled for operation into a $30 \mathrm{~cm} \times 30 \mathrm{~cm}$ thermal column opening. The position of the ISNF cavity inside the reactor thermal column (a $1.4 \times 1.3 \times 0.94$ meter graphite block) is shown in Fig. I-1. A schematic cross section of the ISNF arrangement within the cavity is shown in Fig. I-2. Three levels of access to the irradiation region are available: (1) instruments and irradiation samples may be inserted or removed through a $5 \mathrm{~cm}$ diameter cylindrical channel; (2) the $1{ }^{0} \mathrm{~B}$ shell and graphite pieces to which it is attached slide in a $20 \mathrm{~cm}$ diameter cylindrical access; and (3) the entire cavity block may be withdrawn. Eight $235 \mathrm{U}$ source disks are mounted symmetrically near the surface of the cavity of which four are shown in Fig. I-2.

The flanged cadmium shield fits over the stems of instruments placed inside of the shell and prevents thermal neutrons from streaming through the hole in the shell access plug. Shell access plugs without a hole are used for passive detector irradiations which do not involve instrument stems. Streaming 
through access holes is a characteristic problem for any driven, onedimensional neutron field. Experimental investigation is required to demonstrate that thermal and wall-return neutron streaming is a negligible perturbation.

The critical component of ISNF is the ${ }^{10 \mathrm{~B}}$ shell which establishes the spectrum shape at energies below about $180 \mathrm{keV}$. The shell, formed as stepped hemispheres, at the LoS Alamos National Laboratory by techniques of powder metallurgy is $14.4 \mathrm{~cm}$ outside diameter and $1.29 \mathrm{~cm}$ thick with a total mass of $1389 \mathrm{~g}$ and a mean ${ }^{10} \mathrm{~B}$ thickness of $1.28 \mathrm{~g} / \mathrm{cm}^{2}$. The powder used for fabrication of the shell was a mixture of $92.7 \%$ enriched ${ }^{10} \mathrm{~B}$ metal and al uminum.

The fission source disks of $93 \%$ enriched uranium metal, $16 \mathrm{~mm}$ dia. $\times 0.15 \mathrm{~mm}$ thick, are mounted $1 \mathrm{~cm}$ from the cavity surface. The thermal neutron fluence rate in the cavity is $\sim 2 \times 10^{11} \mathrm{n} /\left(\mathrm{cm}^{2} \mathrm{~s}\right)$ and each disk operates at two watts of fission power. The total fission neutron source strength for the eight disks is $1.1 \times 10^{12} \mathrm{~s}^{-1}$.

\section{3.b. IRRADIATION PROCEDURES}

Irradiation experiments are placed inside of the $10_{B}$ shell with the cavity block outside of the reactor. The block is then inserted into the thermal column and irradiations are initiated by raising the boral curtain behind the thermal column. At the end of the irradiation the curtain is 1 owered and after a suitable cool-down time the block is removed. Alternatively, experiments can be removed by withdrawing the $10 \mathrm{~B}$ shell assembly which is attached to the shell access plug. This procedure leaves the highly radioactive fission disks inside of the thermal column. 
The central region of known spectrum and minimal fluence gradients extends out to a radius of $\sim 2.5 \mathrm{~cm}$ as shown by the dashed circle in Fig. I-2. Neutron fluence monitoring is by means of an activation monitor foil or a fission chamber.

\section{3.c. SPECIFICATION FOR NEUTRON TRANSPORT CALCULATION [Ei77a], [Gr77c], $[\mathrm{MC} 82 \mathrm{a}],[\mathrm{Wa} 81 \mathrm{a}]$}

The ISNF is a spherically symmetric system of $10 \mathrm{~B}$ and carbon with some unavoidable amounts of ${ }^{1} l_{B}$ and aluminum and a minute amount of moisture. The fission source disks which drive the system are mounted $1 \mathrm{~cm}$ from the cavity wal1. Because of spherical symmetry, the fluence contribution at the center from each disk depends only on its source strength and distance from the center, not on its angular position; hence, for the neutron fluence at the center, the eight fission disks considered separately or as a set are equivalent to a shell source.

Material densities and dimensional specifications for purposes of transport calculation are given in Fig. I-3. The two zones shown in the graphite are a one-dimensional representation of the measured density difference between thermal column graphite and the cavity insert blocks - see Fig. I-1. The hydrogen density given for the graphite corresponds to 0.22 weight percent of moisture - see Section 4.b.1. The only significant compromise with the real ISNF system in the calculations is the substitution of carbon for 1 li in the boron shel1. For discrete ordinates, the following computational structure provides sufficient accuracy: 
- Angular quadrature

$\mathrm{S}_{8}$

- Legendre expansion for carbon scatter $P_{3}$

- Spatial mesh

$\begin{array}{lr}\text { vacuum }(0-6 \mathrm{~cm}) & 10 \text { intervals } \\ \text { boron shell }(6-7 \mathrm{~cm}) & 8 \text { intervals } \\ \text { vacuum }(7-15 \mathrm{~cm}) & 9 \text { intervals } \\ \text { graphite }(15-65 \mathrm{~cm}) & 28 \text { intervals }\end{array}$

Spectrum uncertainties associated with errors in densities and dimensions of components are taken up in Section 4.b.

\section{NEUTRON FIELD CHARACTERIZATION}

The ISNF neutron spectrum divides naturally into three energy regions, each dominated by a characteristic feature of the system. For energies above 3.7 MeV, the spectrum resembles that of the $235 \mathrm{U}$ fission neutrons which drive the system; between $0.18 \mathrm{MeV}$ and $3.7 \mathrm{MeV}$, the spectrum is dominated by neutrons returning from the graphite after several collisions; the remainder of the spectrum below $0.18 \mathrm{MeV}$ is determined by $1 / \mathrm{v}$-absorption in the $10_{\mathrm{B}}$ shel1. Partitioned in this way, the ISNF neutron spectrum is as follows:

Three-group characterization

Fission spectrum region ( $>3.7 \mathrm{MeV}$ ): Graphite return region $(0.18-3.7 \mathrm{MeV})$ : $10_{\mathrm{B}}$ absorption region ( $\left.<0.18 \mathrm{MeV}\right)$ :

\begin{tabular}{ccc} 
Group & $235 U(n, f)$ & $10 \mathrm{~B}(n, \alpha)$ \\
response & response \\
fluence & fraction & fraction \\
\hline
\end{tabular}
0.043
0.031
0.013
0.673
0.52
0.22
0.284
0.45
0.77

Spectrum boundaries

$$
\begin{aligned}
& E_{p}(p=0.99)=1.2 \mathrm{keV} \\
& E_{p}(p=0.01)=5.6 \mathrm{MeV}
\end{aligned}
$$

\section{Median energy}

$E_{p}(p=0.50)=0.58 \mathrm{MeV}$ 
Although most of the neutron spectrum 1 ies in the graphite return region, much of the response of a 10 w energy detector 1 ike $235 \mathrm{U}$ fission 1 ies in the $10 \mathrm{~B}$ absorption region. This shift is even more pronounced for a $1 / v$ cross section like $10_{B}(n, \alpha)$.

The complete ISNF spectrum (Fig. I-4) is defined as the 150-group ANISN calculation performed at Los Alamos. The input data are the geometric and physical parameters given in Fig. I-3, the ENDF/B-V cross sections, and for the driving source, an average of the ENDF/B-V and NBS evaluated $235 \mathrm{U}$ fission neutron spectrum shapes. The angular quadrature $\left(S_{8}\right)$ and the spatial mesh for this calculation were shown to be satisfactory in early exploratory computations [Fa74b]. A coarse 14-group energy structure is employed for summarizing the detailed spectrum, for comparisons with other calculations, and for stating spectrum accuracy. The defined central spectrum for ISNF with a thick shell in the 14-group energy structure is given in Table I-1 along with results from three validating calculations.

The large gamma ray intensity in the ISNF cavity comes primarily from thermalneutron capture in graphite. The neutron-to-gamma ratio is about $2 \times 10^{4}\left(\mathrm{n} / \mathrm{cm}^{2} \mathrm{~s}\right) /(\mathrm{R} / \mathrm{h})$.

\section{4.a. NEUTRON FLUENCE}

The ISNF is driven by eight $235 \mathrm{U}$ fission disks which yield a central neutron fluence rate of $\sim 1.5 \times 10^{9} \mathrm{n} / \mathrm{cm}^{2} \mathrm{~s}$, and for a nominal maximum irradiation time of 20 hours, deliver a neutron fluence of $1.1 \times 10^{14} \mathrm{n} / \mathrm{cm}^{2}$. 
The central ISNF fluence is established by means of neutron fluence transfer from the ${ }^{252} \mathrm{Cf}$ Fission Neutron Irradiation Facility (see Section 3.b of Part IB and Refs. [Gr78a], [La84a]). The fluence transfer reaction is $239 \mathrm{pu}(\mathrm{n}, \mathrm{f})$ and the fluence transfer expression is

$$
\Phi_{\text {ISNF }}=\frac{{ }_{C f}\left(>E_{p}\right)}{\sigma_{I S N F}\left(>E_{p}\right)} \cdot \frac{\psi_{C f}\left(>E_{p}\right)}{\psi_{I S N F}\left(>E_{p}\right)} \cdot \frac{D_{I S N F}}{D_{C f}} \cdot \Phi_{C f}
$$

where $\Phi=$ total neutron fluence, and the subscript "ISNF" denotes the central ISNF fluence at the ${ }^{239} \mathrm{Pu}(\mathrm{n}, \mathrm{f})$ monitor position and "Cf" the ${ }^{252} \mathrm{Cf}$ fission neutron fluence associated with the calibration of the $239 \mathrm{pu}(n, f)$ detector.

The ratios on the right side of Eq. (I-I) are, in order, ${ }^{239 p u(n, f)}$ truncated fission cross sections $\sigma\left(>E_{p}\right)$, spectrum fractions $\psi\left(>E_{p}\right)$, and the ratio of the $239 \mathrm{Pu}(n, f)$ reaction rate obtained in the ISNF to that obtained at the ${ }^{252} \mathrm{Cf}$ irradiation facility - see Section 5.a in Part IA. For $239 \mathrm{Pu}(n, f)$ the truncated cross section ratio is 1.031 and the spectrum fraction ratio is 0.952. The error in the product, $1.033 \times 0.952=0.984$, attributable to ${ }^{252} \mathrm{Cf}$ and ISNF spectrum uncertainties is less than $0.3 \%$. However, the $239 \mathrm{pu}(n, f)$ cross section shape error including covariance adds an additional uncertainty of $\pm 1.6 \%$. Combined with a typical error of $\pm 0.8 \%$ for the reaction rate ratio, and $\pm 1.2 \%$ for the ${ }^{252} \mathrm{Cf}$ fission neutron fluence $\Phi_{C f}$, the fluence transfer procedure yields a free-field central ISNF neutron fluence with an accuracy of $\pm 2.1 \%(1 \sigma)$. 
The central ISNF fl uence rate al so has been estimated on the basis of a cursory determination of the neutron source strength of all eight fission disks. This is an involved measurement not amenable to high accuracy. Its purpose was to provide a gross check of the total neutron return fluence in the transport calculation. The assigned ISNF calculation (see Section 4.b.1.(a)) gives a val ue of $1.36 \times 10^{-3} \mathrm{n} / \mathrm{cm}^{2} \mathrm{~s}$ for the central fluence rate per source neutron for this parameter. The measured value is $1.27 \pm 0.07 \times 10^{-3}$.

Fluence variation over $\pm 1.5 \mathrm{~cm}$ of the central region of the ISNF fiel d is less than $\pm 3 \%$. There is some asymmetry in this variation about the geometric center due to unequal source strengths among the fission disks. This slight gradient does not create any spectrum change, and for conventional irradiation experiments carried out with disk-shaped detectors, one of which is a fluence monitor, the uncertainty in the spatial averaged fluence is negligible.

\section{4.b. NEUTRON SPECTRUM}

The fundamental design concept behind ISNF was to create a family of neutron fiel ds whose neutron spectrum could be established entirely on the basis of neutron transport calculation. The following features of the ISNF system were designed to match this concept: (1) simple one dimensional (spherical) geometry; (2) restriction to materials for which neutron cross sections are among the best known; (3) $235 \mathrm{U}$ fission spectrum as the driving source; and (4) spatial decoupling of the el ements which dominate the neutron transport problem (i.e., separation of the neutron source, moderator, and absorber). The arrangement shown in Fig. I-2 consists of a spherical cavity in a 1 arge block of graphite, a shell of $10_{B} 1$ ightly supported at the cavity center, and 
thin fission source disks of $235 \mathrm{U}$ metal placed symmetrically around the periphery of the cavity. Fission neutrons generated in the source disks, both the uncollided and those returning from the graphite, are transmitted by the ${ }^{10} \mathrm{~B}$ shell and give rise to the ISNF field at the center of the cavity. Proper convergence of discrete ordinates calculations for the ISNF geometry and materials is discussed in Refs. [Fa74b] and [Br80a].

The neutron transport problem is dominated by scattering in carbon and absorption in ${ }^{10} \mathrm{~B}$. Therefore, as far as nuclear data is concerned, the neutron spectrum is determined chiefly by the $235 \mathrm{U}$ fission neutron spectrum (above $\sim 3$ MeV only), by the kinematics of elastic scattering in carbon, and by two accurately known cross sections: $\sigma_{\text {tot }}$ for carbon, and $\sigma_{\text {abs }}$ for $10 \mathrm{~B}$. Energy dependence only is required for ${ }^{10} \mathrm{~B}$ absorption because neutron transmission thru the boron shell is determined experimentally in a 2 keV monoenergetic neutron beam. Spectrum calculations in spherical geometry are simple and exact, limited in accuracy only by uncertainties in the input fission spectrum and cross section data. Because of this simplicity, it is easy to investigate the sensitivity of the spectrum to uncertainties in the physical and nuclear parameters of the ISNF system, as well as to scattering in extraneous structural materials.

In the remainder of this section neutron transport calculations will be summarized, an evaluated ISNF spectrum will be described, and spectrum sensitivities to physical and nuclear parameters will be established for various types of detector responses. The evaluated ISNF spectrum is given in Section 4.b.3 along with errors based on a composition of the spectrum sensitivity studies and a quantitative estimate of ISNF parameter uncertainties. 
4.b.1. Calculation. Five neutron transport calculations make up the calculational base for ISNF. Of these, the 150-group calculational result from Los Alamos National Laboratory was chosen for the assigned spectrum. In brief outline the five calculations are as follows:

\section{Assigned}

(a) Discrete ordinates calculation performed at LANL using ONEDANT in the $\mathrm{P}_{3} \mathrm{~S}_{8}$ approximation with a 150-group energy structure and ENDF/B-V cross sections. The angular quadrature $\left(S_{8}\right)$, the spatial mesh (73 space intervals from the center out to $50 \mathrm{~cm}$ into the graphite), and the outer graphite boundary have been investigated for convergence. The assigned spectrum is taken as the average of two calculations, one of which uses the NBS evaluated fission spectrum and the other, the ENDF/B-V fission spectrum shape. The designation ISNF(5)-1-LI refers specifically to this calculation. These calculations are entirely similar to those carried out earlier with ONETRAN (see (e) below) and references for the latter remain applicable [So79b], [La80a].

\section{Supplementary}

(b) Discrete ordinates calculations performed by NBS using ANISNW in the $\mathrm{P}_{3} \mathrm{~S}_{8}$ approximation with a 40-group energy structure [Gr77c]. Groupaveraged cross sections were prepared from ENDF/B-III using SUPERTOG and the GAM-II energy structure [Fa74b]. This calculation is the reference for most of the ISNF sensitivity studies.

(c) Discrete ordinates calculation performed by ORNL using XSDRNPM in the $\mathrm{P}_{3} \mathrm{~S}_{8}$ approximation, ENDF/B-IV cross sections, and the 171-group VITAMIN-C energy structure [Wa81a], [Br80a], [Br79b], [Gr76a]. 
(d) Monte-Carlo calculations performed with ENDF/B-IV cross sections by LANL using MCNP in two options: (i) discrete-reaction and continuousenergy kinematics, and (ii) complete continuous energy [La80a], [So79b], [LA78a].

(e) Early LANL calculations with ONETRAN, 240 energy groups, and the NBS evaluated fission spectrum. Otherwise similar to (a) above [So79b], $[\mathrm{Hi75a}],[\mathrm{La} 80 \mathrm{a}]$.

The assigned spectrum normalized to unit fluence is shown complete in Fig. I-4 and in a 14-group energy structure in Table I-1. Comparisons with the supplementary calculations are also given in the Table and show departures of more than $3 \%$ in some of the energy groups. This may be attributed to the coarse group structure of the NBS calculation and to statistical fluctuations in the Monte Carlo calculation. The comparison of the NBS 40-group calculation with the LANL 150-group illustrates the general insensitivity (except for the lowest energy groups) of spectrum calculations to energy group structure.

Two components of the ISNF spectrum are physically realizable and may be distinguished because of spatial decoupling of the neutron source, the neutron moderator, and the neutron absorber. The first component is the $235 \mathrm{f}$ fission neutron source which drives the system and the second is the neutron return from the graphite plus the fission source neutrons. The second component exists in the ISNF cavity when the boron shell is removed. This empty cavity spectrum relaxes smoothly into a near-1/E spectrum characteristic of a pure scattering medium and as such is also a standard neutron field. Both components are shown in Fig. I-5 along with the composite central ISNF 
spectrum which results from the transmission of the second component through the ${ }^{10_{B}}$ shell. The extent and significance of spectrum decoupling in ISNF is discussed in Ref. [Fa74b].

Sensitivity Studies. The accuracy of the ISNF spectrum is assessed by means of a sensitivity study involving the variation of physical and geometric parameters, input nuclear data for boron and carbon, and the fission spectrum.

Using the 40-group NBS calculation, sensitivity factors are derived for the spectrum itself and for selected spectrum-averaged reaction cross sections. For integral reaction rate measurements, the error associated with the ISNF spectrum is the quadrature sum of the cross section sensitivity factors corresponding to each ISNF parameter uncertainty.

The parameters studied are as follows:

1. Absorption in the $10_{\mathrm{B}}$ shell

[shell thickness $(a t / b)] \times\left[{ }^{10} B\right.$ cross section (b/at) $]$

2. Mean-free-path in graphite:

[graphite density $\times$ carbon total cross section]- 1

3. Anisotropy of carbon scattering

4. Position of source disks

5. Fission spectrum

6. Scattering in the $10_{B}$ shell:

(a) modulation at al uminum resonances; (b) total scatter in ${ }^{27} \mathrm{Al},{ }^{1} \mathrm{~B}_{\mathrm{B}}$, and impurities; (c) high-energy scattering in ${ }^{10} \mathrm{~B}$

7. Scattering in al uminum support structures within the cavity. 
(1) Absorption in 10B shell. The key parameter for determining the shape of the ISNF spectrum below $100 \mathrm{keV}$ is the absorption thickness of $10_{\mathrm{B}}$ in the she11. Uncertainties in the ${ }^{10 B}$ cross section $\sigma(b / a t)$ or the mass thickness $X(a t / b)$ are amplified in the spectrum at 1 ow energies because of the $1 / \mathrm{v}$ behavior of the absorption cross section. The ${ }^{10 B}$ mass thickness for the shell is determined from a measured ${ }^{10_{B}}$ enrichment, the mass of the finished shell, and a chemical analysis of the boron-aluminum powder mix employed. The neutron transmission of the finished shell, measured with a 2 keV filtered neutron beam provides an independent check of the absorption thickness of the shel1. An observed shell transmission of 0.281 was found to be uniform over accessible portions of the shell to better than $1 \%$. The $10_{B}$ mass thickness deduced from the transmission measurement, using ENDF/B-V cross sections for $10 \mathrm{~B}$, is $(3.9 \pm 2) \%$ greater than the thickness obtained from chemical and isotopic analysis and physical weighing. An average of these two values was used for the transport calculations.

The observed neutron transmission is important because it puts into the transport calculation a measured value of $\sigma x$, the neutron absorption in the shell. With the shell transmission established by experiment at one energy, the lowenergy side of the spectrum depends only upon the $1 / v$ behavior of the ${ }^{10} \mathrm{~B}$ absorption which is a cross section standard below $100 \mathrm{keV}$ [Ca84a]. This behavior has been established both by measurement and by theory. From thermal to $1 \mathrm{keV}$ it is accurate to $\pm 1 \%$; up to $10 \mathrm{keV}$ it is within $\pm 2 \%$, and up to $100 \mathrm{keV}$ within $\pm 3 \%[\mathrm{Ca} 84 \mathrm{a}],[\mathrm{Wa} 77 \mathrm{a}],[\mathrm{Ha} 76 \mathrm{a}]$.

The uncertainty in $\sigma X$ for the shell has been set equal to $\pm 2 \%$, the departure of the two mass thickness determinations from the average value. The spectrum 
change associated with a $2 \%$ increase in ${ }^{10} \mathrm{~B}$ thickness is given in the third column of Table I-2. Below about 1 keV the uncertainty in $10 \mathrm{~B}$ thickness is seen to dominate the ISNF spectrum error; above $20 \mathrm{keV}$ it is unimportant.

(2) Mean-free-path in graphite $(1 / \rho \sigma)$. Measured values of graphite density exist for various unused thermal column stringers (standard deviation of $\pm 0.3 \%$ among the densities of four individual pieces), and for two of the cavity assembly pieces. These two sets of densities, stringers and cavity blocks, differ by $4.5 \%$ and are modeled into the one-dimensional calculation. The spectrum difference between the two-zone density model and uniform average graphite density is very smal1. The total cross section for carbon has been studied extensively and its error is below $\pm 0.75 \%$ in the energy region below $0.1 \mathrm{MeV}$ and $\pm 1 \%$ above $0.1 \mathrm{MeV}[\mathrm{He} 75 \mathrm{~b}]$. Based on the density measurements and the cross section data, the uncertainty in the graphite mean-free-path is set conservatively at $\pm 2 \%$ for all energies. The spectrum change associated with a $2 \%$ decrease in mean-free-path is given in the fourth column of Table I-2.

The graphite stringers were also analyzed for moisture by the Karl Fischer titration method. The resulting hydrogen to carbon atom ratio in the graphite from this measurement is $0.003 \pm .0003$. Calculated spectrum differences are listed in column 8 of Table I-2 and corresponds to an estimated error of $\pm 50 \%$ for the in situ moisture content of the graphite in the thermal column. The differences are less than $0.5 \%$ except in the low keV range where they reach $0.8 \%$.

(3) Anisotropy of carbon scattering. Angular distributions of scattering in carbon have been studied in great detail over the energy range of the ISNF 
spectrum and the results have been interpreted by multilevel R-matrix analysis. These investigations make the carbon scattering cross section a reference standard in many experimental studies of neutron interactions [Sm78a]. Angular distributions in discrete ordinates transport calculations are described by Legendre harmonic expansions truncated at $P_{\ell}$. The effect of scattering anisotropy was examined by repeating the calculation with truncation at $P_{3}$ reduced to $P_{0}, P_{1}$, and $P_{2}$. Pure isotropic scattering, the $P_{0}$ approximation, differs from the $\mathrm{P}_{3}$ approximation by more than $10 \%$ in the MeV range of the spectrum and up to $3 \%$ at other energies. The $P_{1}$ component provides some improvement but only with the $P_{2}$ approximation do the departures from the $\mathrm{P}_{3}$ calculation become less than $1.5 \%$ at all energies. The agreement of Monte Carlo calculations with the discrete ordinates calculations (see Table I-1) verifies that scattering in graphite is treated adequately by harmonic expansion in the discrete ordinates computation. The spectrum error from anisotropic scattering in carbon is taken as the spectrum change between the $P_{2}$ and $P_{3}$ approximation. The associated spectrum variation is given in column 7 of Table I-2.

(4) Position of source disk. The enriched uranium metal source disks $(0.5 \mathrm{~g}$ each) are mounted in thin-walled aluminum holders which are press fitted into the graphite wall of the cavity. The uncollided fission neutrons from these disks constitute the largest part of the central fluence above $3 \mathrm{MeV}$. Hence, there is an inverse $-r^{2}$ dependence of the central fast fluence on the position of the sources. The effective source disk position uncertainty is set conservatively at $\pm 1 \mathrm{~mm}$. The spectrum change associated with a decrease of $1 \mathrm{~mm}$ in source radius is given in column 6 of Table $1-2$. 
(5) Fission spectrum Fission neutrons dominate the ISNF spectrum above 3.7 MeV so the error in this energy range is taken to be that of the $235 \mathrm{U}$ fission spectrum. Uncorrelated errors for the NBS fission spectrum evaluation are given in a seven-group energy structure in Table X-5 of Part IA. In order to examine the sensitivity of the entire ISNF spectrum to the fission spectrum it is necessary to have an alternative fission spectrum shape which departs credibly from the NBS evaluation. The fission spectrum shape recommended in the ENDF/B-V file was chosen as an acceptable alternative because the difference is similar to the errors given in Table X-5. A comparison of the NBS and ENDF/B-V evaluations is shown in Table I-3. Separate calculations of ISNF with the two source spectra provide a basis for estimating the ISNF spectrum error attributable to fission spectrum uncertainties.

Time-of-flight measurements have established the average energy of the $235 \mathrm{U}$ spectrum relative to that of ${ }^{252} \mathrm{Cf}[\mathrm{Sm} 79 \mathrm{a}]$. Because the ${ }^{252} \mathrm{Cf}$ spectrum is better known, as indicated by the reduced errors in Table $X-5$, and because the reported difference in average energies in Ref. [Sm79a] agrees better with the NBS evaluation than with the ENDF/B-V value, half the departure of ISNF calculations obtained with these two fission spectra as the neutron source were judged appropriate as a source spectrum error. The spectrum change associated with substituting $x_{B V}$ for $x_{\text {NBS }}$ in the ISNF calculation (divided by 2) is given in column 4 of Table I-4.

(6) Scattering in boron shell.

(6.a) Total scatter in ${ }^{27} \mathrm{Al},{ }^{11} \mathrm{~B}$, and other impurities produce small perturbations in the spectrum. Aluminum, at 26 atom percent of the base material, is the most abundant extraneous material in the she11. An 
uncertainty of $\pm 10 \%$ in the transmission coefficient associated with this amount of aluminum leads to modest spectrum changes in terms of a multigroup description (see column 5 of Table I-2). Localized modulation of the spectrum at aluminum resonances is discussed below. The $1^{1}$ B content at 3 atom percent and other impurities ( $\mathrm{C}, \mathrm{Si}, \mathrm{Fe}$ ) are all entered into the calculation as carbon at 4.5 atom percent. The spectrum uncertainty attributable to these minor constituents is covered by the uncertainty assigned to the al uminum.

(6.b) Modulation of the ISNF spectrum near an aluminum resonance is a striking feature of the ISNF spectrum as displayed in Fig. I-4. This effect, beginning with the resonance at $34 \mathrm{keV}$, is the result of a redistribution of el astically scattered neutrons in a localized energy region. In support of this interpretation, the modulation near an aluminum resonance was reproduced by means of an elementary single scattering analysis involving an aluminum shell of equivalent atoms $/ \mathrm{cm}^{2} \mathrm{placed}$ just inside the boron shell. The scattering is treated as resonance-energy removal of neutrons directed radially inward, and inscatter of the remainder with a spectrum degraded in energy by the average logarithmic decrement for elastic scatter $(\xi=0.072)$. The expression obtained with this analysis is

$$
\frac{\text { ISNF } W / A l}{\operatorname{ISNF} w / O A l}=1-\left[1-e^{-\Sigma_{T}(E) t}\right]+e^{-\Sigma_{T}\left(E^{\prime}\right) t} \Sigma_{e l}\left(E^{\prime}\right) t
$$

where $E^{\prime}=(1+\xi) E$.

Figure I-6 shows the results of this single-scatter analysis for the prominent resonance at $34 \mathrm{keV}$. The smooth ISNF spectrum without aluminum, the negative 
removal contribution, and the positive inscatter contribution are shown separately. The resulting spectrum accurately represents the spectrum calculated by the discrete ordinates method. The difference, indicated by cross hatching in Fig. I- 6 , is only about $1 \%$ of the neutron fluence between 20 $\mathrm{keV}$ and $50 \mathrm{keV}$ or about $0.065 \%$ of the total ISNF fluence. The seven resonances in aluminum which may be distinguished in the ISNF spectrum are at $5.9,34.7,88.5,119.8,145,159$ and $205 \mathrm{keV}$. The effect of this spectrum modulation upon integral reaction rates is small, and the associated error much less, even if there are matching resonances.

(6.c) Scattering in $10 \mathrm{~B}$ above $0.1 \mathrm{MeV}$ has 1 ittle affect as may be seen by comparing ISNF spectra with and without shell in Fig. I-5.

(7) Scattering in aluminum. The support structures within the cavity not included in the specification of ISNF are small. The total mass of the tubes and rings involved is about 40 grams or $6 \%$ of the amount of al uminum in the boron shell.

4.b.2. Measurement. The ISNF is a standard neutron field designed to be characterized by one-dimensional neutron transport calculations. Only in the energy range above $1 \mathrm{MeV}$, where the ISNF is dominated by uncollided fission neutrons, does the spectrum depend upon measurement, namely the fission spectrum itself - see Section 4.b., Part IA. The errors associated with calculation as discussed in the previous section, are smaller than those involved with any type of differential neutron spectrometry. This is particularly true for the spectrum below $50 \mathrm{keV}$, the definition of which is the most distinctive feature of the ISNF system. 
4.b.3. Evaluated Spectrum. The assigned spectrum for ISNF is the average of two Los Alamos 150-group ONEDANT calculations which employ two different $235 \mathrm{U}$ fission spectrum shapes: the ENDF/B-V form and that of the NBS evaluation. A summary of the assigned ISNF spectrum in 14-groups is given in Table I-4 and a graphical display in Figure I-4. The complete 150-group spectrum is available on request.

Spectrum changes associated with errors in all significant nuclear and physical parameters, as discussed in Section 4.b.1, are displayed in Table I-2. The changes are less than $1 \%$ except at extremes of the spectrum. The quadrature sum of these parameter-associated errors for each energy group characterizes the overall accuracy of the spectrum. They are listed in column 3 of Table I-4. The remaining spectrum uncertainty associated with the $235 \mathrm{U}$ fission spectrum source is listed in column 4 of Table I-4 - see Section $4 . b .1(5)$.

The composite group fluence errors set out in Table I-4 indicate a lo bound within which the true ISNF spectrum is estimated to be. For example, the $1 \sigma$ error bound in the energy group 9.12 to $24.8 \mathrm{keV}$ is $\pm \sqrt{(1.14)^{2}+1^{2}}$ or $\pm 1.5 \%$. Such composite errors can aid in establishing the accuracy of a differential spectrometer calibration in ISNF, but they cannot be used to obtain uncertainties in calculated reaction probabilities. In the latter case it is necessary to use the spectrum variations given in Table I-2 because spectrum and parameter changes are fully correlated. The change in reaction cross section associated with each ISNF parameter uncertainty must be established first and then summed in quadrature to obtain a correct reaction rate error. These propagated errors for calculated reaction rates and reaction rate ratios are treated in Section 5.a. 


\section{INTEGRAL DETECTOR RESPONSE}

Neutron dosimetry measurements with integral detectors generally benefit from the use of benchmark neutron fields for cal ibrating measurement techniques and for referencing data interpretation methods. In order to carry out these measurement assurance procedures, and in particular for estimating uncertainties, it is necessary to distinguish between calculated and measured reaction probabilities and to identify response parameters which characterize the energy response of each detector in the various spectra to which it is exposed. A formulation which meets these requirements is outlined in Section 5 of Part IA. Response parameters for a number of integral detectors incl uding cross sections, spectral indexes, and energy response ranges are presented in Section 5.a. Calculated reaction cross section errors, including propagation of spectrum uncertainties, are described in Section 4. Measured reaction probabilities are given in Section 5.b followed by comparison with the calculated values in Section 5.c.

5.a. CALCULATED REACTION PROBABILITIES See Part IA, Section 5.a.

5.a.1. Spectrum Response and Error Propagation Tables. Basic integral detector response parameters for ISNF are given in Table I-5. The spectrum averaged cross sections 1 isted in col umn 2 are the ful 1 -spectrum averaged val ues above a cadmium cut-off energy of $0.4 \mathrm{eV}$ and are followed in col umn 3 by cross sections truncated at a response fraction of $p=0.95$. The spectrum fraction, $\left[\psi\left(>E_{p}\right)\right.$ for $\left.p=0.95\right]$, foll ows in col umn 4. The energy response characteristics of the reaction, given in the last three col umns, are the median response energy and the response range, i.e., the 1 ower- and upperenergy bounds that include $90 \%$ of the detector response. The 1 ower-energy bound, $E_{p}(p=0.95)$, is the truncation energy corresponding to the truncated cross section given in col umn 3 . 
Each of the ISNF parameter errors discussed in Section 4.b.1 corresponds to spectrum variations 1 isted in Table I-2. A convolution of these variations with reaction cross sections in the same multigroup format yields integral cross section errors attributable to the respective ISNF parameter errors. A set of such cross section errors for a representative group of reactions is given in Table I-6. Cross section changes in ISNF which correspond to the fission spectrum difference indicated in Table I-4 (divided by 2) are given in column 3 of Table I-7.

For fission and threshold reactions, it is appropriate to obtain an ISNF response relative to that in the $235 \mathrm{U}$ fission spectrum. The residual error for such a cross section ratio in ISNF, attributable to fission spectrum uncertainties, is the difference between cross section changes associated with uncertainty in a pure fission spectrum (column 4 of Table I-4) and cross section changes in ISNF associated with that same fission spectrum uncertainty (column 3 of Table I-7), all divided by two. This net error is given in column 5 of Table I-7 for fission and threshold reactions. The latter all have more than $50 \%$ of their response above $0.25 \mathrm{MeV}$ in both spectra.

Total propagated errors for calculated ISNF cross sections are presented in Table I-8. The error given in column 4, applicable for absolute cross section measurement, is the quadrature sum of the total error component from the ISNF parameters (column 3 of the table) and the fission spectrum error component from column 3 of Table I-7. The error in column 5 of Table I-8 is for cross section ratios taken relative to the $235 \mathrm{U}$ fission spectrum and is the quadrature sum of the value given in column 3 and that from column 5 of Table 1-7. Errors for reactions not 1 isted will be similar to reactions in the table with the closest median response energy. 
5.a.2. Spectral Indexes. (See Section 5.a.2., Part IA for formulations)

A selected set of spectral indexes calculated for full-spectrum-averaged cross sections from Table I-5 is given in Table I-9; a corresponding set for truncated cross sections is listed in Table I-10.

5.a.3. Spectral Index Errors. The cross section errors associated with individual ISNF parameters given in Table I- 6 are the starting point for spectral index error propagation. The differences between the errors corresponding to the two reactions of the index from Table I- 6 are summed in quadrature and then added to the corresponding error difference from Table I-7 (column 5 if the index is measured relative to the fission spectrum, column 3 if it is not). The summation for the spectral index ${ }^{235} U(n, f) / 238 U(n, f)$ is set out below as an example:

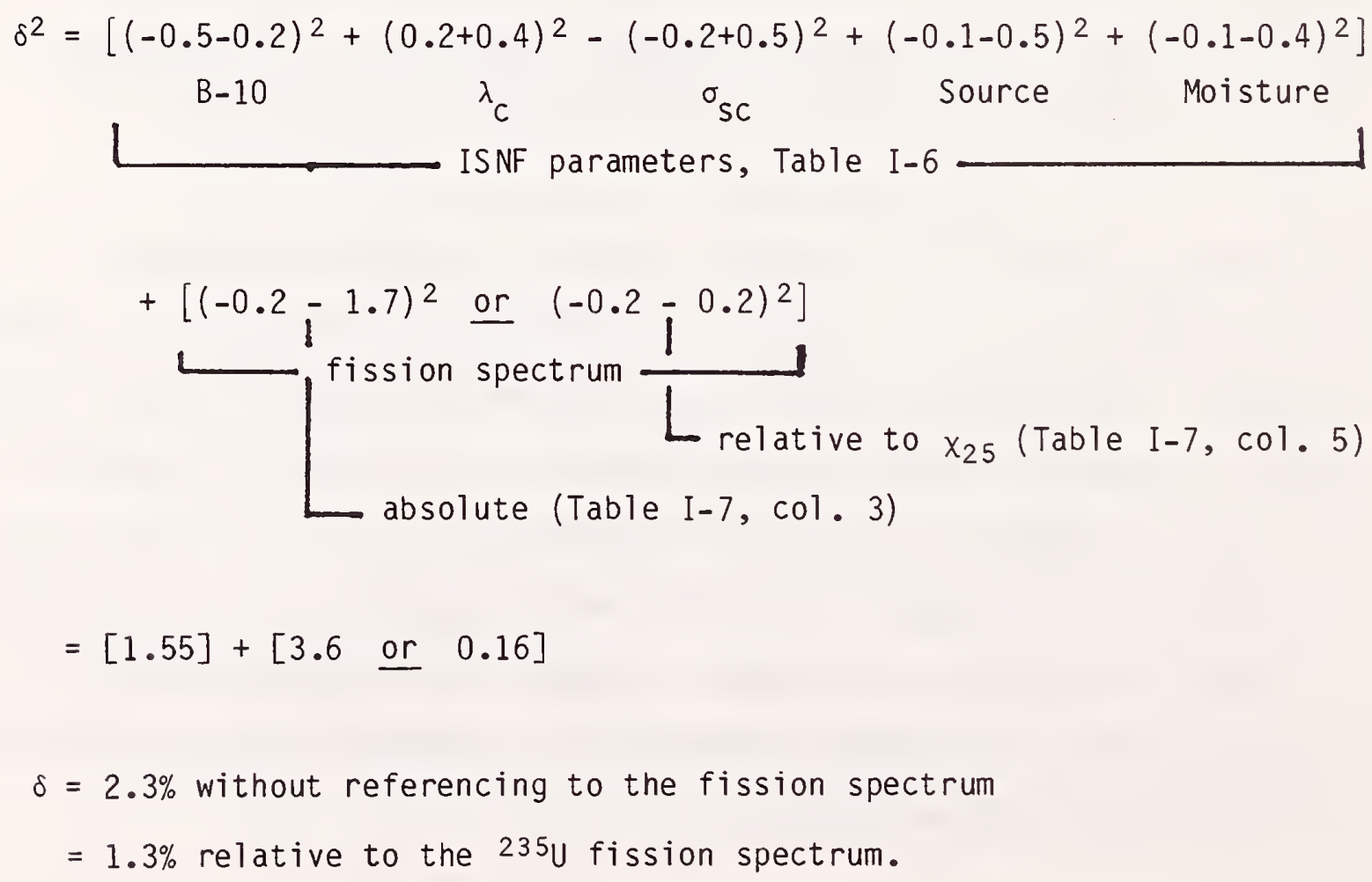


Errors for representative spectral indexes are given in Table I-11. They vary from 1 ess than $\pm 0.5 \%$ to $\pm 3 \%$ depending upon the separation of the spectrum response range of the two detectors and whether the spectral index is taken relative to the fission spectrum. The spectrum associated error combined with experimental uncertainties establish the total error for a test of cross sections by means of a spectral index measurement.

\section{5.b. MEASURED REACTION PROBABILITIES (See Section 5.b, Part IA)}

\section{C. MEASURED CROSS SECTIONS AND CALCULATED-TO-OBSERVED RATIOS}

Most cross section ratio measurements in ISNF have been carried out relative to either the $235 \mathrm{U}$ or the ${ }^{239} 9 \mathrm{pu}$ fission reactions. Results of these measurements have been converted to reaction cross sections based on neutron fluence transfer from the ${ }^{252} \mathrm{Cf}$ Fission Neutron Irradiation Facility employing the $239 \mathrm{pu}(n, f)$ reaction (Section $4 . a)$. Cross sections for seven fissionable isotopes and some significant capture reactions important for dosimetry and nuclear technology are presented in Table I-12.

Threshold reactions show discrepancies with calculated values similar to those observed with fission spectra (cf. Tables X-10(B5) and Table $X-17(B 5)$ ), a result consistent with the fact that the ISNF spectrum is dominated by the fission spectrum in the MeV range. This similarity may be seen by comparing truncated cross sections of threshold detectors in Table $X-13(B 5)$ and Table I-5. The standard deviation of the twelve C/E ratios in Table I-12, excluding $232 \mathrm{Th}$, is 0.028 about an average departure from unity of 1.036 . 
Observed spectral indexes for ISNF are listed in column 2 of Table I-13. In col umn 3, they are given relative to the $235 \mathrm{U}$ fission spectrum. The $235 \mathrm{U}$ fission spectrum values are from Table X-18, Part IB. The C/E ratios for the spectral indexes are shown in Table I-14. The assigned uncertainties represent all error sources except the energy-dependent reaction cross section. Errors associated with the physical and nuclear parameters of ISNF are set out separately in the last two columns of the Table.

For the common fissile materials $239 \mathrm{pu}$ and $235 \mathrm{U}$, calculation and measurement of the fission cross section ratio agree to within $1.5 \%$ indicating consistency of the ENDF/B-V file between $1 \mathrm{keV}$ and $3 \mathrm{MeV}$, the approximate energy response range in ISNF for these two reactions. Poorer agreement for this ratio has been noted for fission spectrum neutrons in Section 5.c, Part IB.

The $C / E$ discrepancy of $5 \%$ for $\mathrm{S}_{\mathrm{U} 8 / \mathrm{U} 5}$, the foremost spectral index in fastneutron technology, is similar to that observed for fission spectra $\left(C / E=0.953 \pm 0.024\right.$ in ISNF vs. $0.946 \pm 0.015$ in $x_{C f}$ and $0.919 \pm 0.022$ in $x_{25}$ ), even though the value of the index in ISNF is very different. 
Table I-1 Fourteen-Group Specification of the ISNF Central Spectrum 4

Table I-2 Spectrum Variation Corresponding to ISNF Parameter Uncertainties

4.b.1

Table I-3 235U Fission Spectrum Errors from Part IA Compared to Difference Between NBS and ENDF/B-V Evaluated Fission Spectra

4.b.3

Table I-4 Assigned ISNF Spectrum and Errors 4.b.3

Table I-5 Integral Detector Response Parameters 5.a.1

Table I-6 Cross Section Changes Associated with Individual ISNF 5.a.1 Parameter Uncertainties

Table I-7 Cross Section Changes Associated with 235U Fission 5.a.1 Spectrum Uncertainty

Table I-8 Total Propagated Error for Calculated ISNF Cross 5.a.1 Sections (1 S.D.)

Table I-9 Calculated Spectral Indexes

5. a.2

Table I-10 Calculated Spectral Indexes--Truncated

5. . . 2

Table I-11 Calculated Spectral Index Errors Associated With

5. a.2 ISNF Spectrum Uncertainties (1 S.D.)

Table I-12 Observed Integral Cross Sections

Table I-13 Observed Spectral Indexes

5.c.

Table I-14 Calculated-to-0bserved Ratios of Spectral Indexes 5.c. 
Table I-1. FOURTEEN-GROUP SPECIFICATION OF ISNF CENTRAL SPECTRUM

Designation: $\operatorname{ISNF}(5)-1-L 1$

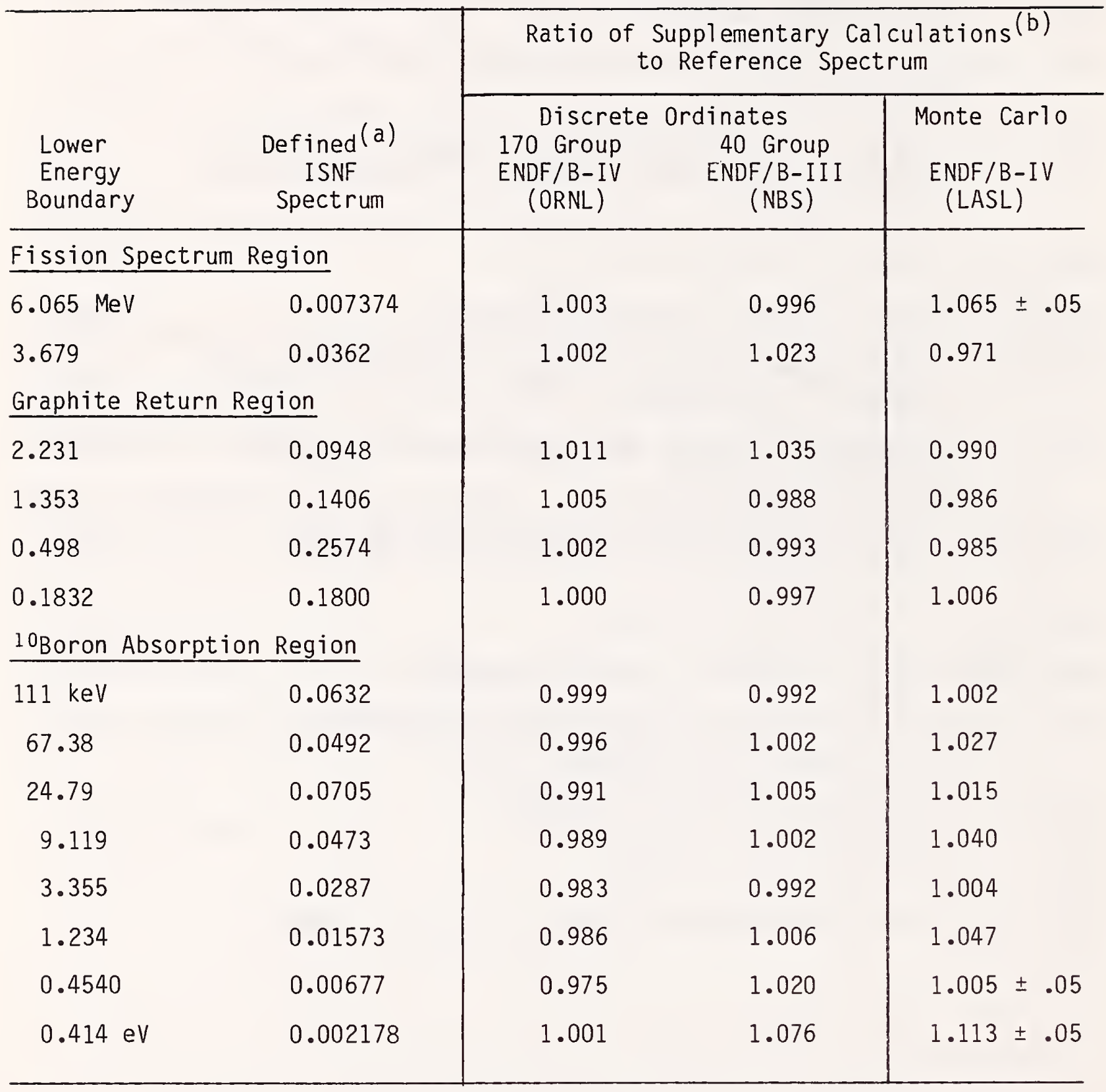

(a) Results of Los Alamos 150-group ONEDANT calculation with ENDF/B-V cross sections (see Section 4.b.1). Spectrum in fine-group structure will be furnished upon request.

(b) The reference spectrum for these calculations is the 240-group LANL spectrum described in section 4.b.1. 


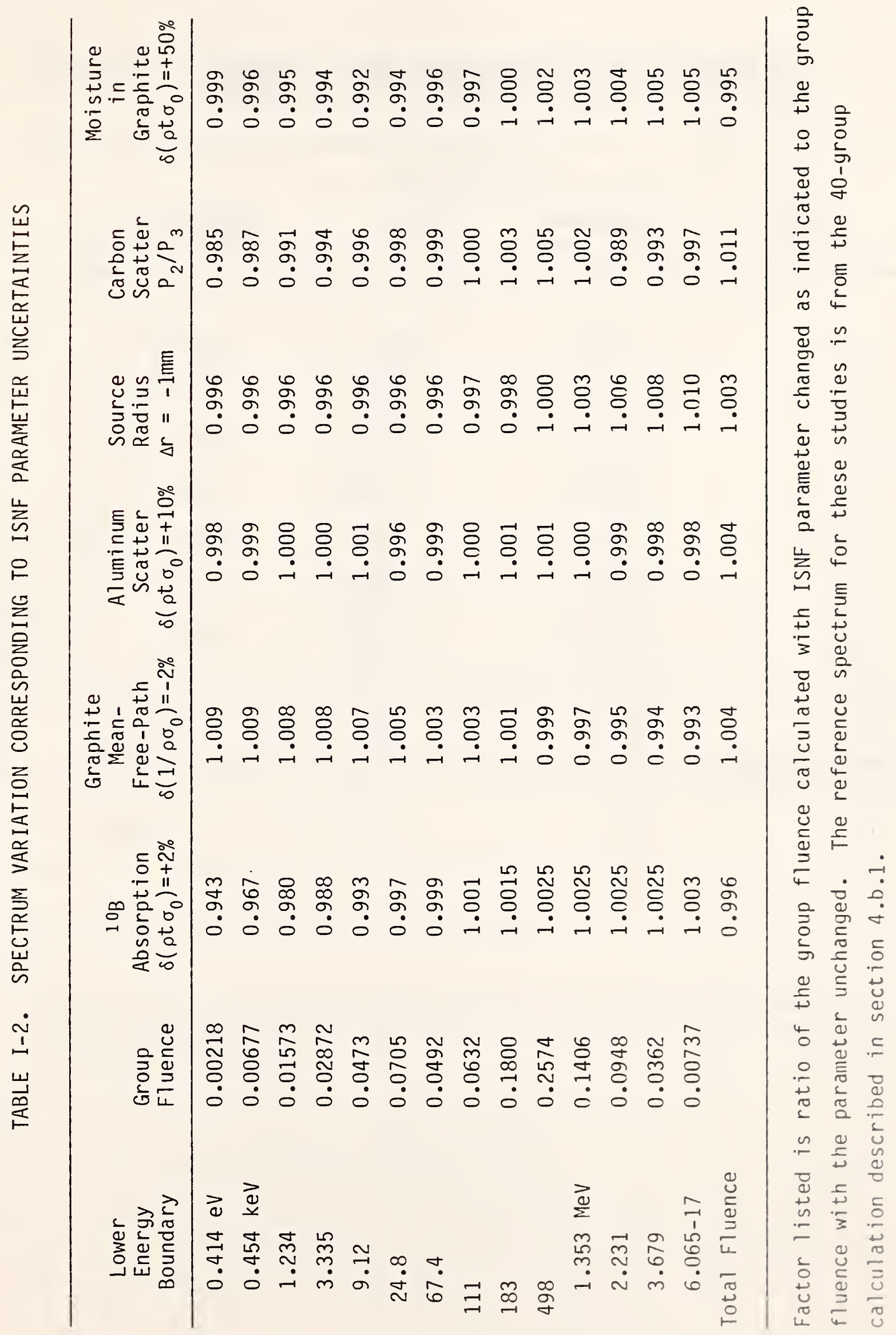


TABLE I-3. 235U FISSION SPECTRUM ERRORS FROM PART IA COMPARED TO DIFFERENCE BETWEEN NBS AND ENDF/B-V EVALUATED FISSION SPECTRA

\begin{tabular}{|c|c|c|c|}
\hline \multirow{2}{*}{$\begin{array}{l}\text { Lower } \\
\text { Energy } \\
\text { Bound }\end{array}$} & \multicolumn{2}{|c|}{ NBS Evaluated } & \multirow[b]{2}{*}{$\frac{\text { ENDF/B-V }}{\text { NBS EvaT. }}$} \\
\hline & Spectrum & $\begin{array}{l}\text { *Error } \\
(1 \sigma)\end{array}$ & \\
\hline 0.0 & 0.054 & $\pm 16 \%$ & 0.992 \\
\hline 0.25 & 0.197 & $\pm \quad 4.1 \%$ & 0.992 \\
\hline 0.8 & 0.229 & $\pm 3.0 \%$ & 1.00 \\
\hline 1.5 & 0.195 & $\pm 3.1 \%$ & 1.026 \\
\hline 2.3 & 0.192 & $\pm 2.0 \%$ & 1.047 \\
\hline 3.7 & 0.127 & $\pm \quad 4.8 \%$ & 1.039 \\
\hline 8 & 0.0056 & $\pm 5.3 \%$ & 1.089 \\
\hline
\end{tabular}

*Error based on departures of measured data sets - see Section $4 . b .3$ and Table $X-5$ in Part IA. 
TABLE I-4. ASSIGNED ISNF SPECTRUM AND ERRORS

\begin{tabular}{llll}
\hline $\begin{array}{c}\text { Lower } \\
\text { Energy } \\
\text { Boundary }\end{array}$ & Spectrum & $\begin{array}{c}\text { Physical (a) } \\
\text { and Nuclear } \\
\text { Parameters }\end{array}$ & $\begin{array}{c}\text { Fission }(b) \\
\text { Spectrum }\end{array}$ \\
\hline & & & \\
$0.414 \mathrm{eV}$ & 0.00218 & $\pm 6.3 \%$ & $\pm 0.6 \%$ \\
$0.454 \mathrm{keV}$ & 0.00677 & \pm 3.7 & $\pm 0.6 \%$ \\
1.234 & 0.01573 & $\pm 2.5 \%$ & $\pm 0.8 \%$ \\
3.355 & 0.0287 & $\pm 2.7 \%$ & $\pm 0.9 \%$ \\
9.12 & 0.0473 & $\pm 1.4 \%$ & $\pm 1.0 \%$ \\
24.8 & 0.0705 & $\pm 1.0 \%$ & $\pm 1.2 \%$ \\
67.4 & 0.0492 & $\pm 0.7 \%$ & $\pm 1.5 \%$ \\
111 & 0.0632 & $\pm 0.5 \%$ & $\pm 1.8 \%$ \\
183 & 0.1800 & $\pm 0.41 \%$ & $\pm 1.5 \%$ \\
498 & 0.2574 & $\pm 0.6 \%$ & $\pm 0.4 \%$ \\
$1.353 \mathrm{MeV}$ & 0.1406 & $\pm 0.6 \%$ & $\pm 1.6 \%$ \\
2.231 & 0.0948 & $\pm 1.4 \%$ & $\pm 2.4 \%$ \\
3.679 & 0.0362 & $\pm 1.4 \%$ & $\pm 2.1 \%$ \\
6.065 to 17 & 0.00737 & $\pm 1.4 \%$ & $\pm 1.4 \%$ \\
\hline
\end{tabular}

(a) Quadrature sum of errors listed in Table I-2.

(b)

Error taken as one half of ISNF spectrum difference corresponding to changing the source spectrum from the NBS evaluated $235 \mathrm{U}$ fission spectrum to that of ENDF/B-V. 
TABLE I-5. INTEGRAL DETECTOR RESPONSE PARAMETERS

Spectrum: Intermediate-Energy Standard Neutron Field (ISNF): Thick Shell

Cross Sections: ENDF/B-V Dosimetry File [EN79a]
Designation: ISNF(5)-1-L1

Entry Date: June, 1983

Revised:

\begin{tabular}{|c|c|c|c|c|c|c|}
\hline $\begin{array}{l}\text { Detector } \\
\text { Reaction }\end{array}$ & $\begin{array}{r}\text { Cross } \\
\sigma(> \\
E_{p}=0.4 e V\end{array}$ & $\begin{array}{l}\text { ction }(a) \\
\frac{p}{p(p=0.95)}\end{array}$ & \begin{tabular}{|} 
Spectrum \\
Fraction \\
$\psi\left(>\mathrm{E}_{95}\right)$
\end{tabular} & $\begin{array}{l}\text { Median }(c) \\
\text { Response } \\
\text { Energy } \\
E_{p}(p=0.5)\end{array}$ & $\frac{\text { Response }}{E_{p}(p=0.95)}$ & $\frac{\operatorname{Range}^{(c)}}{E_{p}(p=0.05)}$ \\
\hline Spectrum Check & (barns) & (barns) & & $(\mathrm{MeV})$ & $(\mathrm{MeV})$ & \\
\hline $\begin{array}{l}\text { Constant } \\
\text { recip. vel., (1/v) (d) }\end{array}$ & $\begin{array}{l}1.000 \\
0.348\end{array}$ & $\begin{array}{l}1.000 \\
0.332\end{array}$ & $\begin{array}{l}0.950 \\
0.998\end{array}$ & $\begin{array}{ll}1.59 \\
2.47 & E-2\end{array}$ & $\begin{array}{l}8.3 E-3 \\
4.9 E-4\end{array}$ & $\begin{array}{l}3.49 \\
1.6\end{array}$ \\
\hline 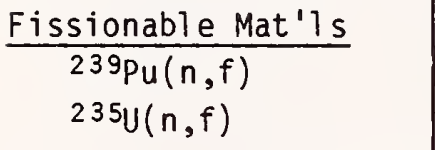 & $\begin{array}{l}1.821 \\
1.602\end{array}$ & $\begin{array}{l}1.750 \\
1.531\end{array}$ & $\begin{array}{l}0.989 \\
0.994\end{array}$ & $\begin{array}{l}0.58 \\
0.26\end{array}$ & $\begin{array}{l}1.5 E-3 \\
9.0 E-4\end{array}$ & $\begin{array}{l}3.5 \\
3.1\end{array}$ \\
\hline $\begin{array}{l}233 U(n, f) \\
238 U(n, f)\end{array}$ & $\begin{array}{l}2.373 \\
0.1416\end{array}$ & $\begin{array}{l}2.275 \\
0.507\end{array}$ & $\begin{array}{l}0.991 \\
0.266\end{array}$ & $\begin{array}{l}0.33 \\
2.33\end{array}$ & $\begin{array}{l}1.2 E-3 \\
1.4\end{array}$ & $\begin{array}{l}3.1 \\
5.9\end{array}$ \\
\hline $\begin{array}{l}237 \mathrm{~Np}(n, f) \\
232 \operatorname{Th}(n, f)\end{array}$ & $\begin{array}{l}0.803 \\
3.37 E-2\end{array}$ & $\begin{array}{l}1.464 \\
0.1221\end{array}$ & $\begin{array}{l}0.521 \\
0.2617\end{array}$ & $\begin{array}{l}1.54 \\
2.4\end{array}$ & $\begin{array}{l}0.53 \\
1.4\end{array}$ & $\begin{array}{l}4.5 \\
6.6\end{array}$ \\
\hline $\begin{array}{l}240 \mathrm{Pu}(n, f) \\
24 \mathrm{lpu}(n, f)\end{array}$ & $\begin{array}{l}0.824 \\
2.102\end{array}$ & $\begin{array}{l}1.338 \\
2.010\end{array}$ & $\begin{array}{l}0.585 \\
0.994\end{array}$ & $\begin{array}{l}1.52 \\
0.26\end{array}$ & $\begin{array}{l}0.39 \\
9.4 \quad E-4\end{array}$ & $\begin{array}{l}4.4 \\
3.0\end{array}$ \\
\hline $\begin{array}{l}238 U(n, \gamma) \\
232 \operatorname{Th}(n, \gamma)\end{array}$ & $\begin{array}{l}0.2215 \\
0.2517\end{array}$ & $\begin{array}{l}0.2107 \\
0.2394\end{array}$ & $\begin{array}{l}0.999 \\
0.999\end{array}$ & $\begin{array}{l}0.023 \\
0.031\end{array}$ & $\begin{array}{l}3.4 \quad E-4 \\
3.2 E-4\end{array}$ & $\begin{array}{l}1.3 \\
1.5\end{array}$ \\
\hline $\begin{array}{c}\text { Capture Reactions } \\
23 \mathrm{Na} \\
45 \mathrm{SC} \\
58 \mathrm{Fe}\end{array}$ & $\begin{array}{l}1.886 E-3 \\
2.707 E-2 \\
7.06 \quad E-3\end{array}$ & $\begin{array}{ll}1.815 & E-3 \\
2.631 & E-2 \\
6.717 & E-3\end{array}$ & $\begin{array}{l}0.987 \\
0.977 \\
0.999\end{array}$ & $\begin{array}{l}2.9 E-3 \\
1.75 \quad E-2 \\
1.03 \quad E-2\end{array}$ & $\begin{array}{ll}1.7 & E-3 \\
3.0 & E-3 \\
3.4 & E-4\end{array}$ & $\begin{array}{l}0.71 \\
0.88 \\
1.3\end{array}$ \\
\hline $\begin{array}{l}{ }^{59} \mathrm{Co} \\
{ }^{63} \mathrm{Cu}\end{array}$ & $\begin{array}{ll}4.09 & E-2 \\
5.13 & E-2\end{array}$ & $\begin{array}{l}3.883 E-2 \\
4.89 E-2\end{array}$ & $\begin{array}{l}1.000 \\
0.997\end{array}$ & $\begin{array}{l}1.42 E-4 \\
5.3 E-3\end{array}$ & $\begin{array}{l}1.23 E-4 \\
5.8 E-4\end{array}$ & $\begin{array}{l}0.94 \\
1.06\end{array}$ \\
\hline $\begin{array}{l}115 \mathrm{In} \\
197 \mathrm{Au}\end{array}$ & $\begin{array}{l}0.2838 \\
0.3924\end{array}$ & $\begin{array}{l}0.2702 \\
0.3733\end{array}$ & $\begin{array}{l}0.998 \\
0.999\end{array}$ & $\begin{array}{l}5.2 \mathrm{E}-2 \\
6.5 \mathrm{E}-3\end{array}$ & $\begin{array}{l}4.7 E-4 \\
3.3 E-4\end{array}$ & $\begin{array}{l}1.8 \\
0.97\end{array}$ \\
\hline
\end{tabular}


TABLE I-5. INTEGRAL DETECTOR RESPONSE PARAMETERS (Continued)

Spectrum: Intermediate-Energy Standard Neutron Field (ISNF): Thick Shell

Cross Sections: ENDF/B-V Dosimetry File [EN79a]
Designation: $\operatorname{ISNF}(5)-1-L 1$

Entry Date: June, 1983

Revised:

\begin{tabular}{|c|c|c|c|c|c|c|}
\hline \multirow[t]{2}{*}{$\begin{array}{l}\text { Detector } \\
\text { Reaction }\end{array}$} & \multicolumn{2}{|c|}{$\begin{array}{c}\text { Cross } \operatorname{Section}(a) \\
\sigma\left(>E_{p}\right)\end{array}$} & \multirow{2}{*}{$\begin{array}{l}\text { Spectrum }(b) \\
\text { Fraction } \\
\psi\left(>E_{95}\right)\end{array}$} & \multirow[t]{2}{*}{$\begin{array}{l}\text { Median }(c) \\
\text { Response } \\
\text { Energy } \\
E_{p}(p=0.5)\end{array}$} & Response & \multirow{2}{*}{$\frac{\operatorname{Range}^{(c)}}{E_{p}(p=0.05)}$} \\
\hline & $E_{p}=0.4 e V$ & $E_{p}(p=0.95)$ & & & $E_{p}(p=0.95)$ & \\
\hline Helium Production & (barns) & (barns) & & $(\mathrm{MeV})$ & $(\mathrm{MeV})$ & $(\mathrm{MeV})$ \\
\hline${ }^{10} B(n, \alpha)$ & 1.667 & 1.587 & 0.998 & $2.03 E-2$ & $4.7 E-4$ & 1.8 \\
\hline${ }^{6} \operatorname{Li}(n, \alpha)$ & 0.7887 & 0.7535 & 0.995 & 0.205 & $8.4 E-4$ & 3.3 \\
\hline Threshold Reactions & & & & & & \\
\hline${ }^{115} \operatorname{In}\left(n, n^{\prime}\right)$ & $8.66 E-2$ & 0.2283 & 0.360 & 2.28 & 1.0 & 5.3 \\
\hline${ }^{47} \mathrm{Ti}(\mathrm{n}, \mathrm{p})$ & $8.62 E-3$ & $3.816 \mathrm{E}-2$ & 0.2145 & 3.33 & 1.7 & 7.0 \\
\hline${ }^{32} S(n, p)$ & $2.552 \mathrm{E}-2$ & 0.1707 & 0.1403 & 3.69 & 2.2 & 7.0 \\
\hline${ }^{58} \mathrm{Ni}(\mathrm{n}, \mathrm{p})$ & $3.80 \mathrm{E}-2$ & 0.1954 & 0.1845 & 3.73 & 1.9 & 7.2 \\
\hline${ }^{54} \mathrm{Fe}(n, p)$ & $2.845 \mathrm{E}-2$ & 0.1840 & 0.1469 & 3.87 & 2.2 & 7.3 \\
\hline${ }^{46} \mathrm{Ti}(n, p)$ & $3.37 \mathrm{E}-3$ & $7.50 \mathrm{E}-2$ & $4.27 E-2$ & 5.6 & 3.7 & 9.2 \\
\hline${ }^{27} A \ell(n, p)$ & $1.293 \mathrm{E}-3$ & $2.333 \mathrm{E}-2$ & $5.27 \mathrm{E}-2$ & 5.6 & 3.4 & 9.2 \\
\hline${ }^{56} \mathrm{Fe}(\mathrm{n}, \mathrm{p})$ & $2.963 E-4$ & $2.353 \mathrm{E}-2$ & $1.196 \mathrm{E}-2$ & 7.3 & 5.4 & 11 \\
\hline${ }^{63} \mathrm{Cu}(n, \alpha)$ & $1.608 \mathrm{E}-4$ & $7.13 E-3$ & $2.143 \mathrm{E}-2$ & 7.3 & 4.7 & 11 \\
\hline${ }^{27} \mathrm{Al}(\mathrm{n}, \alpha)$ & $2.016 E-4$ & $3.51 \quad E-2$ & $5.46 \quad E-3$ & 8.4 & 6.5 & 12 \\
\hline${ }^{48} \mathrm{Ti}(\mathrm{n}, \mathrm{p})$ & $7.95 E-5$ & $8.93 E-3$ & $8.46 E-3$ & 8.1 & 5.9 & 12 \\
\hline Additions (e) & & & & & & \\
\hline${ }^{60} \mathrm{Ni}(n, p)$ & 7.53 E-4 & $3.79 E-2$ & $1.890 \mathrm{E}-2$ & 6.9 & 4.8 & 10 \\
\hline${ }^{55} \operatorname{Mn}(n, 2 n)$ & $5.64 \mathrm{E}-5$ & 3.54 E-1 & $1.513 \mathrm{E}-4$ & 13 & 11 & 16 \\
\hline $103 R h\left(n, n^{\prime}\right)$ & 0.390 & 0.689 & 0.538 & 1.76 & 0.49 & 4.9 \\
\hline${ }^{93} \mathrm{Nb}\left(n, n^{\prime}\right)$ & 0.0810 & 0.1793 & 0.429 & 2.18 & 0.79 & 4.9 \\
\hline dpa & 500 & 667 & 0.712 & 1.8 & 0.19 & 5.3 \\
\hline
\end{tabular}


(a) The value given in column 2 is the full-spectrum-averaged cross section above a cadmium cut-off of $0.4 \mathrm{eV}$. The truncated cross section in column 3 is for a truncation energy (column 6) above which $95 \%$ of the detector response occurs. A spectrum-averaged cross section truncated at energy $E_{p}$ is given by

$$
\sigma\left(>E_{p}\right)=\int_{E_{p}}^{\infty} \sigma(E) \psi(E) d E / \int_{E_{p}}^{\infty} \psi(E) d E
$$

(b) The fraction of the spectrum above $E_{95} \equiv E_{p}(p=0.95): \psi\left(>E_{95}\right)=$ $\int_{E_{95}}^{\infty} \psi(E) d E$. The full-spectrum-averaged cross section $\sigma(>0.4 \mathrm{eV})$ is equal
to $\sigma\left(>E_{95}\right) \cdot \psi\left(>E_{95}\right) / 0.95$.

(c) The fractions $p=0.95,0.5$, and 0.05 define energies above which $95 \%, 50 \%$ (median), and $5 \%$ of the detector response occurs, respectively. $E_{p}$ is defined by the relation

$$
\int_{E_{p}}^{\infty} \sigma(E) \psi(E) d E=p \cdot[\sigma(>0.4 \mathrm{eV})]
$$

where $E_{p}(p=1)=0.4 \mathrm{eV}$, and $E(p=0)=20 \mathrm{MeV} ;$ and $\int_{0.4 \text { eV }}^{\infty} \psi(E) d E=1$.

(d) Normal ization is $\int_{0.4 \mathrm{ev}}^{18} \sigma_{1 / \mathrm{v}} \mathrm{MeV} \psi(E) \mathrm{dE}=1$

(e) Cross sections not taken from the ENDF/BV Dosimetry File:

- $\mathrm{Nb}\left(n, n^{\prime}\right)$ is from the IRDF Dosimetry File [Cu80a].

- $\operatorname{Rh}\left(n, n^{\prime}\right)$ is from data reported in Ref. [Pa80a].

- dpa, the atom displacement cross section, is from ASTM Standard Practice E693-79 [An83a]. 


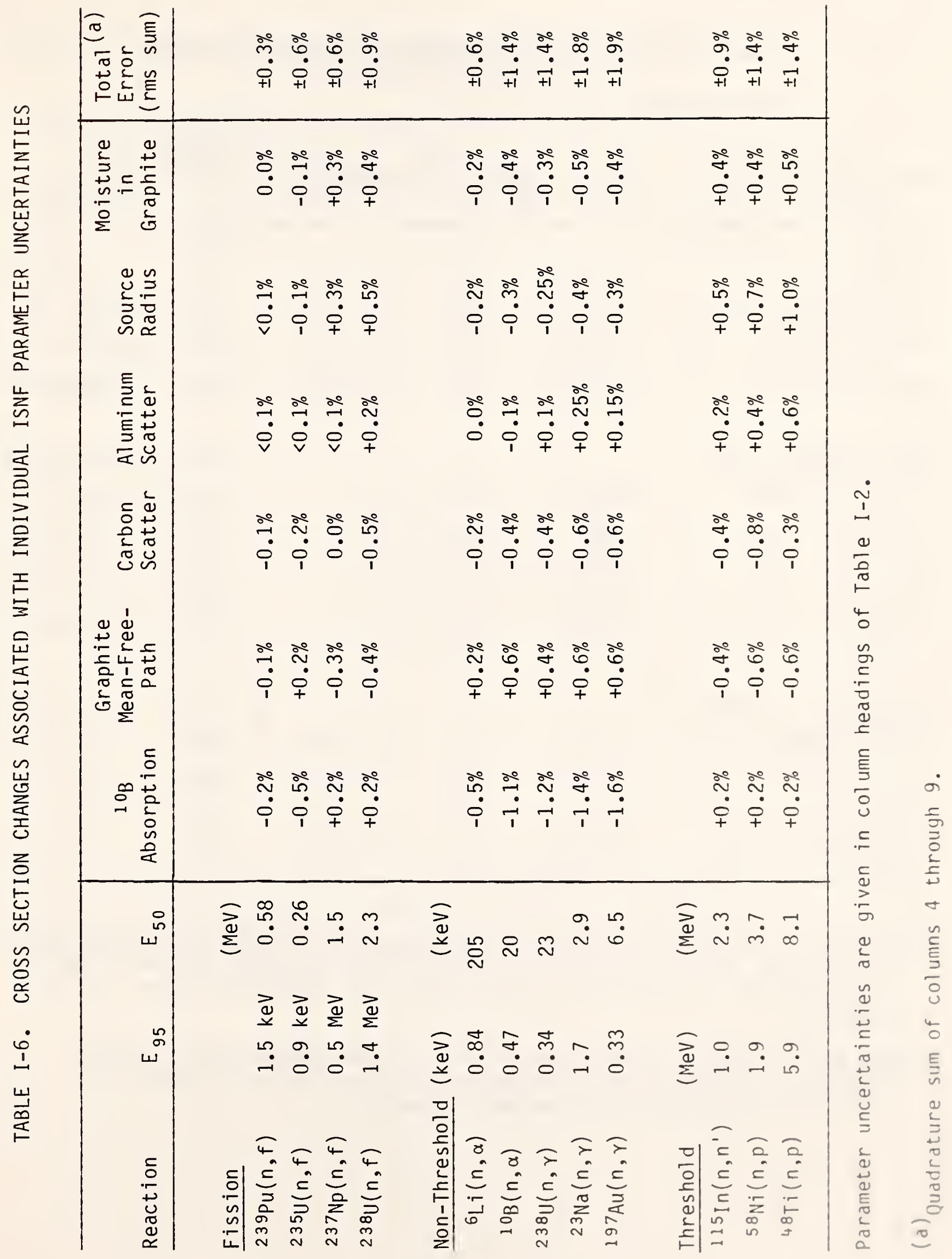


TABLE I-7. CROSS SECTION CHANGES ASSOCIATED WITH 235U

FISSION SPECTRUM UNCERTAINTY

\begin{tabular}{|c|c|c|c|c|c|c|}
\hline \multirow[b]{2}{*}{ Reaction } & \multirow[b]{2}{*}{$E_{50}$} & \multicolumn{3}{|c|}{ Cross Section Change } & \multicolumn{2}{|c|}{$\begin{array}{l}\text { Response Fraction } \\
\text { Above } 0.25 \mathrm{MeV} \\
\end{array}$} \\
\hline & & $\operatorname{ISNF}(a)$ & $\begin{array}{l}\text { Fission(D) } \\
\text { Spectrum }\end{array}$ & $\frac{\text { ISNF }}{\text { Fiss. Spec. }}$ & ISNF & $\begin{array}{l}\text { Fission } \\
\text { Spectrum }\end{array}$ \\
\hline Fission & $(\mathrm{MeV})$ & & & & & \\
\hline $239 \mathrm{pu}(\mathrm{n}, f)$ & 0.6 & $+0.1 \%$ & $+0.1 \%$ & $<0.1 \%$ & 0.64 & 0.95 \\
\hline $235 U(n, f)$ & 0.26 & $-0.2 \%$ & $0.0 \%$ & $-0.2 \%$ & 0.51 & 0.93 \\
\hline $237 \mathrm{~Np}(n, f)$ & 1.5 & $+1.0 \%$ & $+0.9 \%$ & $<0.2 \%$ & 0.99 & 1.00 \\
\hline $238 U(n, f)$ & 2.3 & $+1.7 \%$ & $+1.8 \%$ & $<0.2 \%$ & 1.00 & 1.009 \\
\hline \multicolumn{7}{|c|}{ Non-Threshold (keV) } \\
\hline${ }^{6} \operatorname{Li}(n, \alpha)$ & 205 & -0.7 & $(-1.1 \%)$ & & 0.39 & 0.83 \\
\hline $10 B(n, \alpha)$ & 20 & -0.6 & $(-0.9 \%)$ & & 0.19 & 0.80 \\
\hline $238 U(n, \gamma)$ & 23 & $-0.5 \%$ & $(-1.3 \%)$ & & 0.25 & 0.86 \\
\hline $197 \mathrm{Au}(\mathrm{n}, \gamma)$ & 6.5 & $-0.6 \%$ & $(-1.7 \%)$ & & 0.14 & 0.79 \\
\hline${ }^{23} \mathrm{Na}(\mathrm{n}, \gamma)$ & 2.9 & $-0.5 \%$ & $(-1.2 \%)$ & & 0.097 & 0.75 \\
\hline Threshold & $(\mathrm{MeV})$ & & & & & \\
\hline${ }^{115} \operatorname{In}\left(n, n^{\prime}\right)$ & 2.3 & $+1.6 \%$ & $+1.7 \%$ & $<0.2 \%$ & 1.00 & 1.00 \\
\hline${ }^{58} \mathrm{Ni}(n, p)$ & 3.7 & $+1.8 \%$ & $+2.0 \%$ & $-0.2 \%$ & 1.00 & 1.00 \\
\hline${ }^{46} \mathrm{Ti}(\mathrm{n}, \mathrm{p})$ & 5.6 & $+1.4 \%$ & $+1.6 \%$ & $-0.2 \%$ & 1.00 & 1.00 \\
\hline${ }^{48} \mathrm{Ti}(n, p)$ & 8.1 & $+1.6 \%$ & $+1.7 \%$ & $<0.2 \%$ & 1.00 & 1.00 \\
\hline
\end{tabular}

(a) One half of departure from unity in percent of the cross section obtained with an ISNF calculation using the ENDF/B-V fission spectrum divided by the cross section obtained with a calculation using the NBS-evaluated fission spectrum.

(b) One half of departure from unity of the fission spectrum cross section obtained using the ENDF/B-V fission spectrum evaluation divided by the cross section obtained with the NBS evaluation. 
TABLE I-8. TOTAL PROPAGATED ERROR FOR CALCULATED ISNF CROSS SECTIONS ( 1 S.D.)

\begin{tabular}{|c|c|c|c|c|}
\hline Reaction & $\begin{array}{l}\text { Median } \\
\text { Response } \\
\text { Energy } \\
\left(E_{50}\right)\end{array}$ & $\begin{array}{l}\text { Total Error } \\
\text { Component From } \\
\text { ISNF Parameters } \\
(\text { Table I-6) }\end{array}$ & $\begin{array}{l}\text { Total Pro } \\
\text { Absolute } \\
\text { Cross Section }\end{array}$ & $\begin{array}{l}\text { gated Error } \\
\text { Cross Section (b) } \\
\text { Rel ative to } \\
\text { Fission Spectrum }\end{array}$ \\
\hline Fission & $(\mathrm{MeV})$ & & & \\
\hline $239 \mathrm{pu}$ & 0.6 & $\pm 0.3 \%$ & $\pm 0.3 \%$ & $\pm 0.3 \%$ \\
\hline $235 \mathrm{U}, 233 \mathrm{U}$ & 0.3 & $\pm 0.6 \%$ & $\pm 0.6 \%$ & $\pm 0.6 \%$ \\
\hline $237 \mathrm{~Np}, \quad 240 \mathrm{Pu}$ & 1.5 & $\pm 0.6 \%$ & $\pm 1.2 \%$ & $\pm 0.6 \%$ \\
\hline${ }^{238} \mathrm{U}, \quad 232 \mathrm{Th}$ & 2.3 & $\pm 1.0 \%$ & $\pm 2.0 \%$ & $\pm 1.0 \%$ \\
\hline \multicolumn{5}{|c|}{ Non-Threshold (keV) } \\
\hline${ }^{6} \operatorname{Li}(n, \alpha)$ & 205 & $\pm 0.6 \%$ & $\pm 0.9 \%$ & - \\
\hline${ }^{10} B(n, \alpha)$ & 20 & $\pm 1.4 \%$ & $\pm 1.5 \%$ & - \\
\hline $238 U(n, \gamma)$ & 23 & $\pm 1.4 \%$ & $\pm 1.5 \%$ & - \\
\hline${ }^{197} \mathrm{Au}(\mathrm{n}, \gamma)$ & 6.5 & $\pm 1.9 \%$ & $\pm 2.0 \%$ & - \\
\hline${ }^{23} \mathrm{Na}(\mathrm{n}, \gamma)$ & 2.9 & $\pm 1.8 \%$ & $\pm 1.9 \%$ & - \\
\hline Threshold & $(\mathrm{MeV})$ & & & \\
\hline${ }^{115} \operatorname{In}\left(n, n^{\prime}\right)$ & 2.3 & $\pm 0.9 \%$ & $\pm 1.8 \%$ & $\pm 0.9 \%$ \\
\hline${ }^{58} \mathrm{Ni}(n, p)$ & 3.7 & $\pm 1.4 \%$ & $\pm 2.3 \%$ & $\pm 1.4 \%$ \\
\hline${ }^{46} \mathrm{Ti}(n, p)$ & 5.6 & & & \\
\hline${ }^{48} \mathrm{Ti}(\mathrm{n}, \mathrm{p})$ & 8.1 & $\pm 1.4 \%$ & $\pm 2.1 \%$ & $\pm 1.4 \%$ \\
\hline
\end{tabular}


TABLE I-9. CALCULATED SPECTRAL INDEXES

Spectrum: Intermediate-Energy Standard Neutron Field (ISNF): Thick Shell

Designation: $\operatorname{ISNF}(5)-1-L 1$ Entry Date: June, 1983

Spectral Index: Fu1 1-Spectrum Cross Sections from

Table I-5 (ENDF/B-V Dosimetry File) Revised:

$$
\left[s_{\alpha / \beta}\right]_{\text {calc. }} \equiv\left[\bar{\sigma}_{\alpha} / \bar{\sigma}_{\beta}\right]_{c a l c .}
$$

\begin{tabular}{|c|c|c|c|c|}
\hline$\alpha \beta$ & $\begin{array}{l}235 u(n, f) \\
=1.602 b)\end{array}$ & $\begin{array}{c}238 U(n, f) \\
(\bar{\sigma}=0.1416 b)\end{array}$ & $\alpha \beta$ & $\begin{array}{c}238 \mathrm{U}(\mathrm{n}, \mathrm{f}) \\
(\bar{\sigma}=0.1416 \mathrm{~b})\end{array}$ \\
\hline \multicolumn{3}{|l|}{ Fission } & \multicolumn{2}{|l|}{ Threshold } \\
\hline $239 \mathrm{Pu}$ & 1.137 & 12.86 & ${ }^{115} \operatorname{In}\left(n, n^{\prime}\right)$ & 0.612 \\
\hline $235 u$ & 1.000 & 11.31 & ${ }^{47} T i(n, p)$ & 0.0609 \\
\hline $233 U$ & 1.481 & 16.76 & ${ }^{58} \mathrm{Ni}(n, p)$ & 0.268 \\
\hline $238 \mathrm{U}$ & 0.0884 & 1.000 & ${ }^{54} \mathrm{Fe}(n, p)$ & 0.2009 \\
\hline $237 \mathrm{~Np}$ & 1.501 & 5.67 & ${ }^{46} T i(n, p)$ & 0.02380 \\
\hline $240 \mathrm{pu}$ & 0.514 & 5.82 & ${ }^{56} \mathrm{Fe}(\mathrm{n}, \mathrm{p})$ & 0.002093 \\
\hline \multicolumn{3}{|l|}{ Non-Threshold } & ${ }^{63} \mathrm{Cu}(n, \alpha)$ & 0.001136 \\
\hline${ }^{6} \operatorname{Li}(n, \alpha)$ & 0.492 & 5.57 & ${ }^{48} T i(n, p)$ & $5.61 E-4$ \\
\hline${ }^{10} B(n, \alpha)$ & 1.041 & 11.77 & ${ }^{27} \mathrm{Al}(n, \alpha)$ & 0.001424 \\
\hline $238 U(n, \gamma)$ & 0.1383 & 1.564 & Additions & \\
\hline${ }^{232} \operatorname{Th}(n, \gamma)$ & 0.1571 & 1.778 & $10{ }^{3} \operatorname{Rh}\left(n, n^{\prime}\right)$ & 2.754 \\
\hline${ }^{197 \mathrm{Au}(n, \gamma)}$ & 0.2449 & 2.771 & ${ }^{93} \mathrm{Nb}\left(n, n^{\prime}\right)$ & 0.572 \\
\hline${ }^{59} \operatorname{Co}(n, \gamma)$ & 0.02553 & 0.2888 & & \\
\hline${ }^{58} \mathrm{Fe}(\mathrm{n}, \gamma)$ & 0.00441 & 0.0499 & & \\
\hline${ }^{23} \mathrm{Na}(n, \gamma)$ & 0.001178 & 0.01332 & & \\
\hline
\end{tabular}


TABLE I-10. CALCULATED SPECTRAL INDEXES -- TRUNCATED

Spectrum: Intermediate-Energy Standard Neutron Field (ISNF): Thick Shell

Spectral Index: Truncated Cross Sections from

Designation: $\operatorname{ISNF}(5)-1-L 1$

Entry Date: June, 1983

Table I-5 (ENDF/B-V Dosimetry File) Revised:

$\left[S_{\alpha / \beta}\right]_{c a l c .} \equiv\left[\bar{\sigma}_{\alpha}\left(>E_{95}\right) / \bar{\sigma}_{\beta}\left(>E_{95}\right)\right]_{c a l c}$.

\begin{tabular}{|c|c|c|c|c|}
\hline$\beta$ & $\begin{array}{c}238 U(n, f) \\
\bar{\sigma}\left(>E_{95}\right)=0.507 b\end{array}$ & $\begin{array}{c}{ }^{237} \mathrm{~Np}(\mathrm{n}, \mathrm{f}) \\
\bar{\sigma}\left(>\mathrm{E}_{95}\right)=1.464 \mathrm{~b}\end{array}$ & $\alpha$ & $\begin{array}{c}238 U(n, f) \\
\bar{\sigma}\left(>E_{95}\right)=0.507 b\end{array}$ \\
\hline Fission & & & Threshold & \\
\hline $239 \mathrm{Pu}$ & 3.452 & 1.195 & ${ }^{115} \operatorname{In}\left(n, n^{1}\right)$ & 0.450 \\
\hline $235 U$ & 3.020 & 1.046 & ${ }^{47} \mathrm{Ti}(\mathrm{n}, \mathrm{p})$ & 0.0753 \\
\hline $233 U$ & 4.49 & 1.553 & ${ }^{58} \mathrm{Ni}(n, p)$ & 0.385 \\
\hline $238 \mathrm{U}$ & 1.000 & 0.346 & ${ }^{54} \mathrm{Fe}(\mathrm{n}, \mathrm{p})$ & 0.363 \\
\hline $237 \mathrm{~Np}$ & 2.888 & 1.000 & ${ }^{46} \mathrm{Ti}(n, p)$ & 0.1479 \\
\hline $232 \mathrm{Th}$ & 0.2408 & 0.0833 & ${ }^{56} \mathrm{Fe}(n, p)$ & 0.0464 \\
\hline \multirow[t]{6}{*}{$240 \mathrm{Pu}$} & 2.639 & 0.913 & ${ }^{63} \mathrm{Cu}(n, \alpha)$ & 0.01406 \\
\hline & & & ${ }^{48} \mathrm{Ti}(\mathrm{n}, \mathrm{p})$ & 0.01761 \\
\hline & & & ${ }^{27} \mathrm{Al}(\mathrm{n}, \alpha)$ & 0.0692 \\
\hline & & & Additions & \\
\hline & & & $103 R h\left(n, n^{\prime}\right)$ & 1.359 \\
\hline & & & ${ }^{93} \mathrm{Nb}\left(n, n^{\prime}\right)$ & 0.354 \\
\hline
\end{tabular}


TABLE I-11. CALCULATED SPECTRAL INDEX ERRORS ASSOCIATED WITH ISNF SPECTRUM UNCERTAINTIES (1 S.D.)

Propagated Error(a)

Spectral Index

Without

Normalization to

Fission Spectrum
With

Normalization to

Fission Spectrum

\section{Fission}

$235 \mathrm{U} / \mathrm{B}$
$(\beta=239 \mathrm{Pu}, 233 \mathrm{U})$

$235 \mathrm{U} / \mathrm{B}$

$\left(\beta=238 \mathrm{U},{ }^{232} \mathrm{Th}\right)$

$235 \mathrm{U} / 237 \mathrm{~Np}$

$237 \mathrm{~Np} / 238 \mathrm{U}$

Non-Threshold

$$
\begin{aligned}
& { }^{6} \operatorname{Li}(n, \alpha) / 235 U(n, f) \\
& { }^{10} B(n, \alpha) / 235 U(n, f) \\
& 238 U(n, \gamma) / B \\
& (\beta=235 U, 239 P u, 233 U \text { fission) } \\
& { }^{197} \mathrm{Au}(n, \gamma) / B \\
& (B=235 U, 239 P u, 233 U \text { fission) } \\
& 238 U(n, \gamma) / 238 U(n, f)
\end{aligned}
$$

Threshold

$$
\begin{array}{llr}
{ }^{115} \operatorname{In}\left(n, n^{\prime}\right) / 238 U(n, f) & \pm 0.3 \% & \pm 0.2 \% \\
{ }^{58} \mathrm{Ni}(n, p) / 238 U(n, f) & \pm 0.5 \% & \pm 0.5 \% \\
{ }^{58} \mathrm{Ni}(n, p) / 239 \mathrm{Pu}(n, f) & \pm 2.1 \% & \pm 1.3 \% \\
{ }^{48} \mathrm{Ti}(n, p) / 238 U(n, f) & \pm 0.7 \% & \pm 0.7 \%
\end{array}
$$

(a) Algebraic sum of corresponding errors in Table I-6 and I-7 added in quadrature--see Section 5.a.2. 
TABLE I-12. OBSERVED INTEGRAL CROSS SECTIONS

Spectrum: Intermediate-Energy Standard Neutron Field (ISNF): Thick Shell
Designation: $\operatorname{ISNF}(5)-1-L 1$

Entry Date: June, 1983

Revised: July, 1985

\begin{tabular}{cccc}
\hline \multirow{3}{*}{ Reaction } & $\begin{array}{c}\text { Median } \\
\text { Response } \\
\text { Energy, } \\
\mathrm{E}_{50}(\mathrm{MeV})\end{array}$ & $\begin{array}{c}\text { Cross Section } \\
\text { Value } \\
\left(\times 10^{27} \mathrm{~cm}^{2}\right)\end{array}$ & $\begin{array}{c}\text { Calculated-(b) } \\
\text { to-0bserved }\end{array}$ \\
\hline
\end{tabular}

\section{Fission}

\begin{tabular}{|c|c|c|c|c|c|}
\hline $235 \mathrm{U}$ & 0.26 & 1606 & $\pm 2.2 \%$ & $0.998 \pm 2.3 \%$ & {$[$ Gr83a], [Wa81a] } \\
\hline $233 \mathrm{U}$ & 0.33 & 2424 & $\pm 2.7 \%$ & $0.980 \pm 2.8 \%$ & {$[\operatorname{Gr} 83 a]$} \\
\hline $238 U$ & 2.33 & 149.0 & $\pm 2.4 \%$ & $0.950 \pm 3.1 \%$ & {$[$ Gr83a], [Wa81a] } \\
\hline${ }^{237} \mathrm{~Np}$ & 1.54 & 829 & $\pm 2.7 \%$ & $0.969 \pm 3.0 \%$ & {$[$ Gr83a], [Wa81a] } \\
\hline $232 \mathrm{Th}$ & 2.4 & 38.4 & $\pm 3.2 \%$ & $0.878 \pm 3.8 \%$ & [Gr83a] \\
\hline $240 \mathrm{Pu}$ & 1.52 & 824 & $\pm 2.8 \%$ & $1.000 \pm 3.0 \%$ & {$[\mathrm{Gr} 83 \mathrm{a}],[\mathrm{Mc} 82 \mathrm{a}]$} \\
\hline $241 \mathrm{Pu}$ & 0.26 & 2152 & $\pm 5.0 \%$ & $0.977 \pm 5.0 \%$ & [Gr83a] \\
\hline
\end{tabular}

Non-Threshold

\begin{tabular}{|c|c|c|c|c|c|}
\hline $10^{0}(n, H e)$ & 0.020 & 1831 & $\pm 3.3 \%$ & $0.910 \pm 3.6 \%$ & {$[$ Gr85a], [0184a] } \\
\hline${ }^{6} \mathrm{Li}(\mathrm{n}, \mathrm{He})$ & 0.205 & 831 & $\pm 3.0 \%$ & $0.949 \pm 3.1 \%$ & {$[\operatorname{Gr} 85 a],[0184 a]$} \\
\hline $238 U(n, \gamma)$ & 0.023 & 227 & $\pm 2.6 \%$ & $0.976 \pm 3.0 \%$ & [Gi79a] \\
\hline${ }^{232} \operatorname{Th}(n, \gamma)$ & 0.031 & 265 & $\pm 3.6 \%$ & $0.950 \pm 3.9 \%$ & {$[\mathrm{Gi} 79 \mathrm{a}]$} \\
\hline${ }^{197} \mathrm{Au}(\mathrm{n}, \gamma)$ & 0.0065 & 398 & $\pm 3.4 \%$ & $0.986 \pm 3.7 \%$ & [Fa79a] \\
\hline
\end{tabular}

Threshold
${ }^{115} \operatorname{In}\left(n, n^{\prime}\right)$
2.3
$91.8 \pm 3.0 \%$
$0.943 \pm 3.5 \%$
$[\mathrm{Fa79a}]$
(a) Derived from cross section ratios of Table I-13 and fluence transfer from ${ }^{252} \mathrm{Cf}$ Fission Neutron Irradiation Facility. The fluence transfer error is $\pm 2.1 \%$--see Section 4. .

(b) Calculated cross section from Table I-5 (ENDF/B-V) with error from column 4 of Table I-8 combined in quadrature with cross section error in column 3. 
TABLE I-13. OBSERVED SPECTRAL INDEXES

Spectrum: Intermediate-Energy Standard Neutron Field (ISNF): Thick Shel 1
Designation: $\operatorname{ISNF}(5)-1-L 1$

Entry Date: June, 1983

Revised: July, 1985

$\left[s_{\alpha / \beta}\right]_{\text {obs }}=\left[\bar{\sigma}_{\alpha} / \bar{\sigma}_{\beta}\right]_{\text {obs }}$

\begin{tabular}{|c|c|c|c|}
\hline Reaction & $\begin{array}{l}\text { Spectral }(a) \\
\text { Index } \\
{\left[S_{\alpha / \beta}\right]_{\text {ISNF }}}\end{array}$ & $\begin{array}{l}\text { Spectral Index }(\mathrm{b}) \\
\text { Relative to } 235 \mathrm{U} \\
\text { Fission Spectrum } \\
{\left[\mathrm{S}_{\alpha / \beta}\right]_{\text {ISNF }} /\left[\mathrm{S}_{\alpha / \beta}\right]_{\mathrm{X}_{235}}}\end{array}$ & References \\
\hline \multicolumn{4}{|l|}{ Fission } \\
\hline $235 \mathrm{U} /{ }^{239} \mathrm{Pu}$ & $0.866 \pm 1.0 \%$ & $1.312 \pm 1.4 \%$ & [Wa81a] \\
\hline $233 U / 235 U$ & $1.509 \pm 1.6 \%$ & $0.930 \pm 2.1 \%$ & {$[\mathrm{Gr} 83 \mathrm{a}]$} \\
\hline $238 \mathrm{U} / 235 \mathrm{U}$ & $0.0928 \pm 1.1 \%$ & $0.357 \pm 1.2 \%$ & [Wa81a] \\
\hline $237 \mathrm{~Np} / 235 \mathrm{U}$ & $0.516 \pm 1.6 \%$ & $0.456 \pm 1.8 \%$ & [Wa81a] \\
\hline $240 \mathrm{Pu} / 235 \mathrm{U}$ & $0.513 \pm 1.8 \%$ & $0.463 \pm 2.1 \%$ & [Mc82a] \\
\hline $241 \mathrm{Pu} / 235 \mathrm{U}$ & $1.340 \pm 4.6 \%$ & - & [Gr83a] \\
\hline \multicolumn{4}{|l|}{ Non-Threshold } \\
\hline${ }^{10} \mathrm{~B}(\mathrm{n}, \mathrm{He}) /{ }^{235} U(n, f)$ & $1.141 \pm 2.7 \%$ & - & {$[\mathrm{Gr} 85 \mathrm{a}]$} \\
\hline${ }^{6} \mathrm{Li}(\mathrm{n}, \mathrm{He}) /{ }^{235} U(\mathrm{n}, \mathrm{f})$ & $0.518 \pm 2.3 \%$ & - & {$[\mathrm{Gr} 85 \mathrm{a}]$} \\
\hline${ }^{238} U(n, \gamma) / 235 U(n, f)$ & $0.1412 \pm 1.7 \%$ & - & [Gi79a] \\
\hline${ }^{23} \operatorname{Th}(n, \gamma) /{ }^{235} U(n, f)$ & $0.165 \pm 3 \%$ & - & [Gi79a] \\
\hline${ }^{197} \mathrm{Au}(n, \gamma) /{ }^{235} U(n, f)$ & $0.248 \pm 2.9 \%$ & - & [Fa79a $]$ \\
\hline \multicolumn{4}{|l|}{ Threshold } \\
\hline${ }^{115} I n\left(n, n^{\prime}\right) / 238 U(n, f)$ & $0.616 \pm 2.1 \%$ & $1.011 \pm 2.9 \%$ & {$[\mathrm{Fa} 79 \mathrm{a}]$} \\
\hline
\end{tabular}

(a) Observed values obtained with activation detectors or an NBS double fission chamber.

(b) Spectral indexes for the $235 \mathrm{U}$ fission spectrum are from Table $\mathrm{X}-18$, Part IB. 
TABLE I-14 CALCULATED-TO-OBSERVED RATIOS OF SPECTRAL INDEXES

Spectrum: Intermediate-Energy Standard Neutron Field (ISNF): Thick Shell

Designation: $\operatorname{ISNF}(5)-1-L 1$

Entry Date: June 1983

Revised: July, 1985

\begin{tabular}{|c|c|c|c|c|}
\hline \multirow[b]{2}{*}{ Reactions } & \multicolumn{2}{|c|}{ Calcul ated-to-0bserved } & \multirow{2}{*}{\multicolumn{2}{|c|}{$\begin{array}{c}\text { Spectrum-Associated } \\
\text { Error in } \\
\text { Calculated Index }\end{array}$}} \\
\hline & $\begin{array}{c}\text { (a) Without } \\
\text { Normal ization to } \\
235 \mathrm{U} \\
\text { Fission Spectrum }\end{array}$ & $\begin{array}{c}\text { (b) With } \\
\text { Normalization to } \\
235 \mathrm{U} \\
\text { Fission Spectrum }\end{array}$ & & \\
\hline Fission & & & w/o norm & w/norm \\
\hline $235 \mathrm{U} / 239 \mathrm{pu}$ & $1.015 \pm 1.1 \%$ & $0.969 \pm 1.5 \%$ & $< \pm 0.5 \%$ & $< \pm 0.5 \%$ \\
\hline $233 U / 235 U$ & $0.981 \pm 1.7 \%$ & $1.029 \pm 2.2 \%$ & $< \pm 0.5 \%$ & $< \pm 0.5 \%$ \\
\hline $238 U / 235 U$ & $0.953 \pm 2.5 \%$ & $1.039 \pm 1.9 \%$ & $\pm 2.3 \%$ & $\pm 1.4 \%$ \\
\hline $237 \mathrm{~Np} / 235 \mathrm{U}$ & $0.971 \pm 2.1 \%$ & $1.027 \pm 2.1 \%$ & $\pm 1.4 \%$ & $\pm 1.0 \%$ \\
\hline $240 \mathrm{Pu} / 235 \mathrm{U}$ & $1.002 \pm 2.3 \%$ & $1.035 \pm 2.3 \%$ & $\pm 1.4 \%$ & $\pm 1.0 \%$ \\
\hline $\begin{array}{l}24 \mathrm{lPu} / 235 \mathrm{U} \\
\text { Non-Threshold } \\
\end{array}$ & $0.979 \pm 4.6 \%$ & - & $< \pm 0.5 \%$ & \\
\hline${ }^{10} B(n, \alpha) / 235 U(n, f)$ & $0.912 \pm 2.9 \%$ & - & $\pm 0.9 \%$ & - \\
\hline${ }^{6} \operatorname{Li}(n, \alpha) /{ }^{235} U(n, f)$ & $0.950 \pm 2.4 \%$ & - & $\pm 0.5 \%$ & - \\
\hline${ }^{238} U(n, \gamma) /{ }^{235} U(n, f)$ & $0.979 \pm 2.0 \%$ & - & $\pm 1.0 \%$ & - \\
\hline${ }^{23}{ }^{2} T h(n, \gamma) / 235 U(n, f)$ & $0.952 \pm 3.2 \%$ & - & $\pm 1.0 \%$ & - \\
\hline${ }^{197} \mathrm{Au}(n, \gamma) / 235 U(n, f)$ & $0.988 \pm 3.2 \%$ & - & $\pm 1.4 \%$ & - \\
\hline Threshold & & & & \\
\hline${ }^{115} I n\left(n, n^{\prime}\right) / 238 U(n, f)$ & $0.994 \pm 2.1 \%$ & $1.029 \pm 3.7 \%$ & $\pm 0.3 \%$ & $\pm 0.2 \%$ \\
\hline
\end{tabular}

(a) Calculated spectral index from Table I-9; observed value from Table I-13, column 2. Includes ISNF spectrum error from column 4.

(b) Calculated spectral indexes from Table I-9 (ISNF) and Table $X-14(B 5)$, Part IB; observed spectral indexes from Table I-13, column 3. Includes spectrum error from column 5 . 


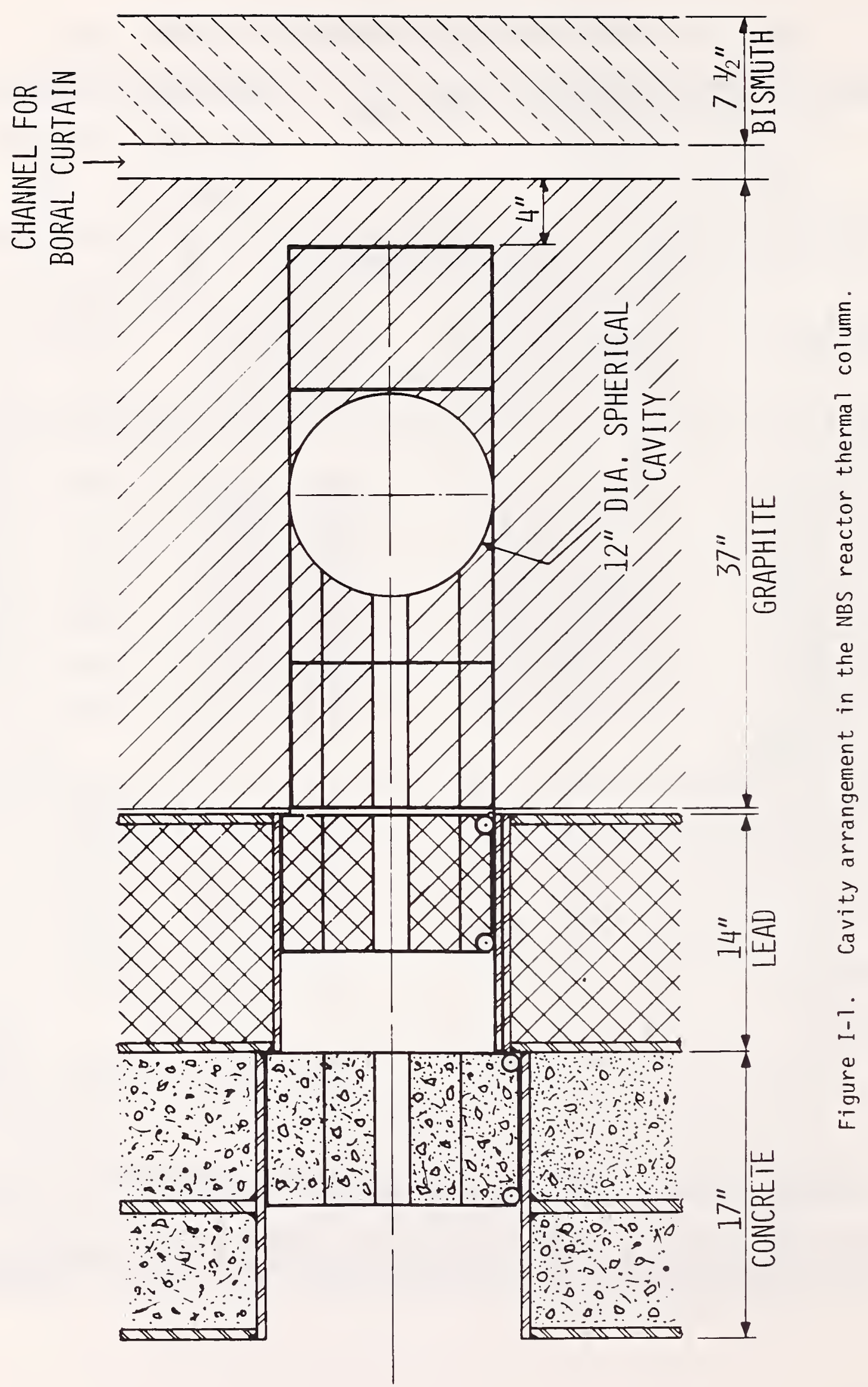



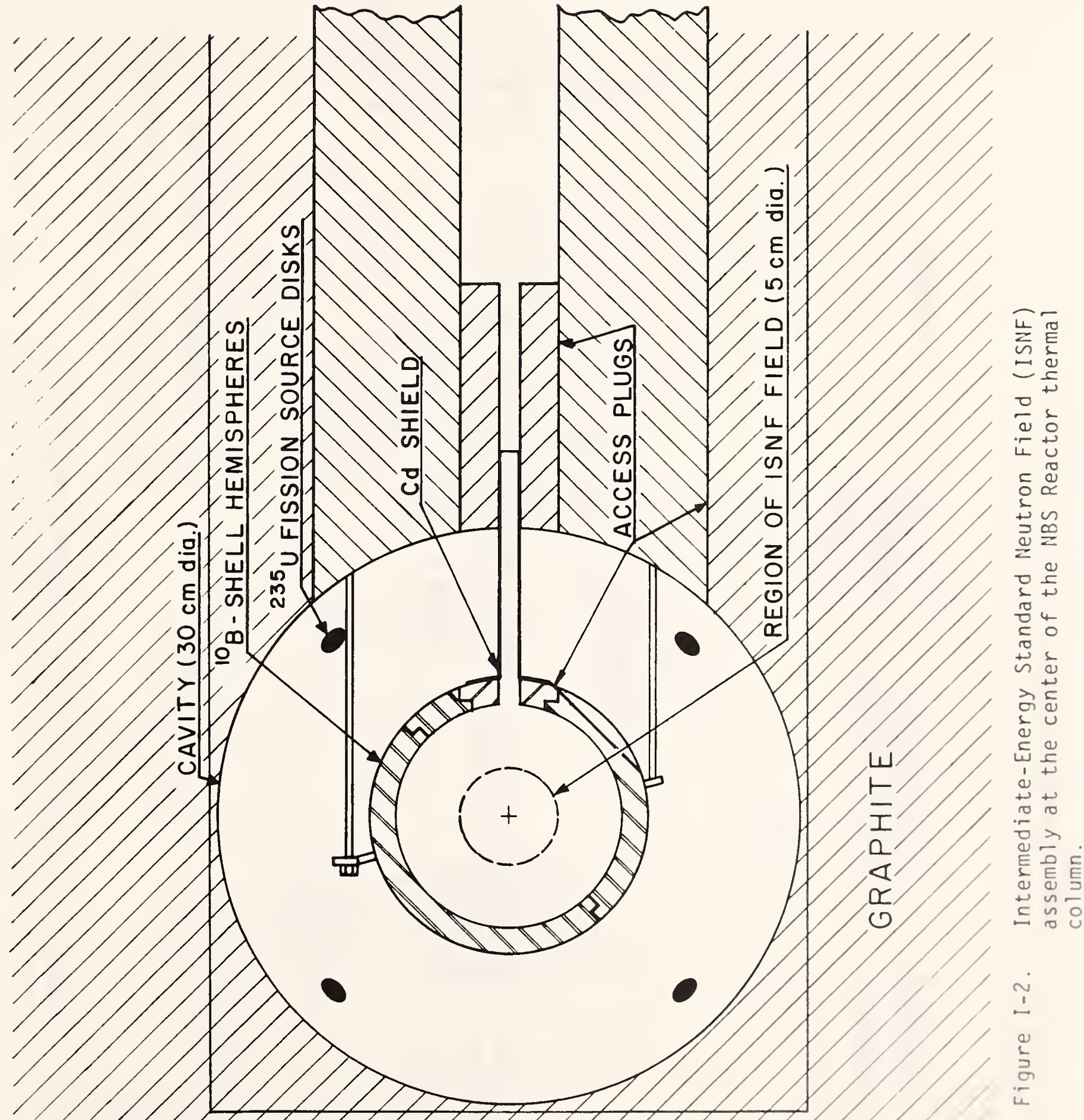


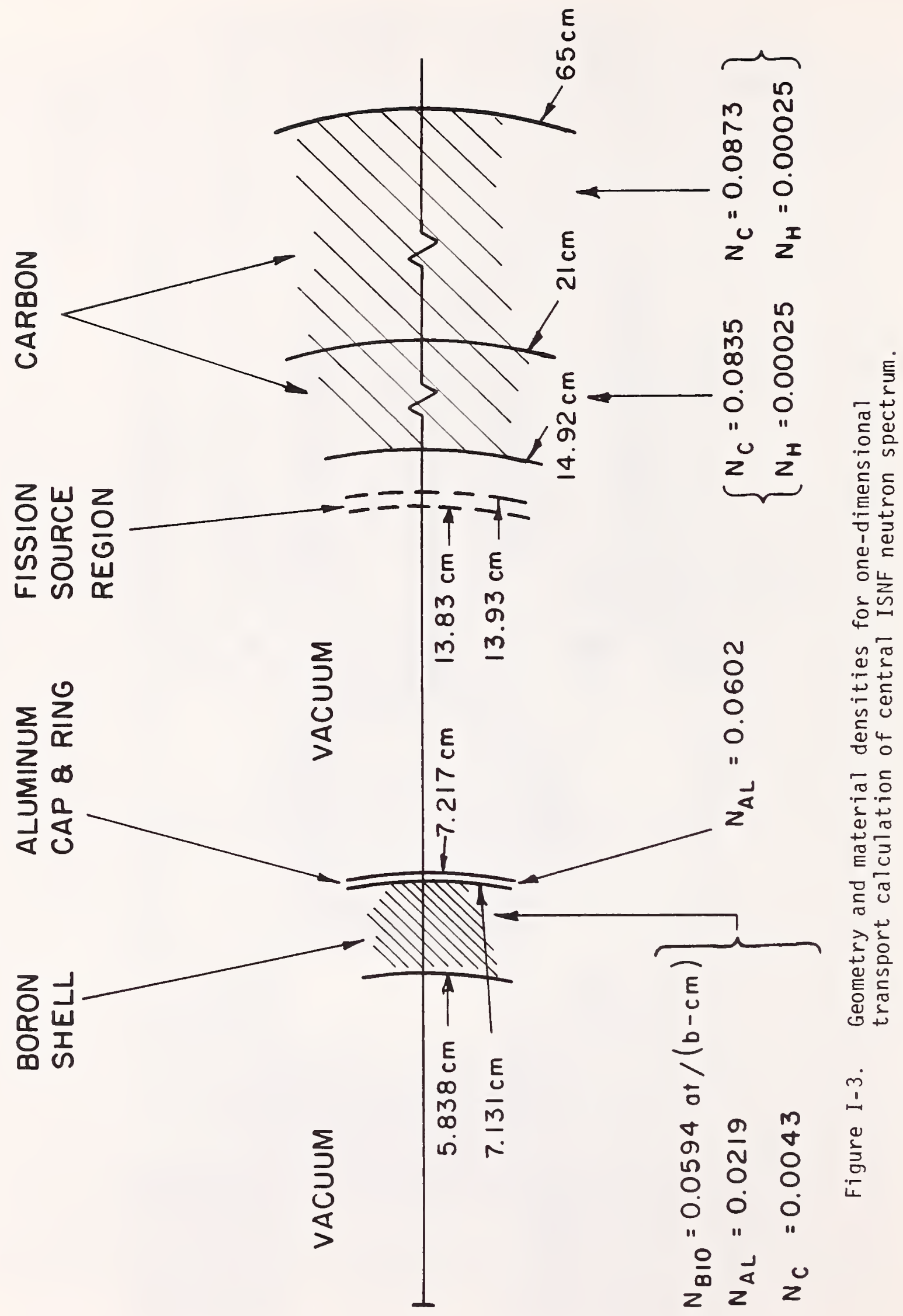




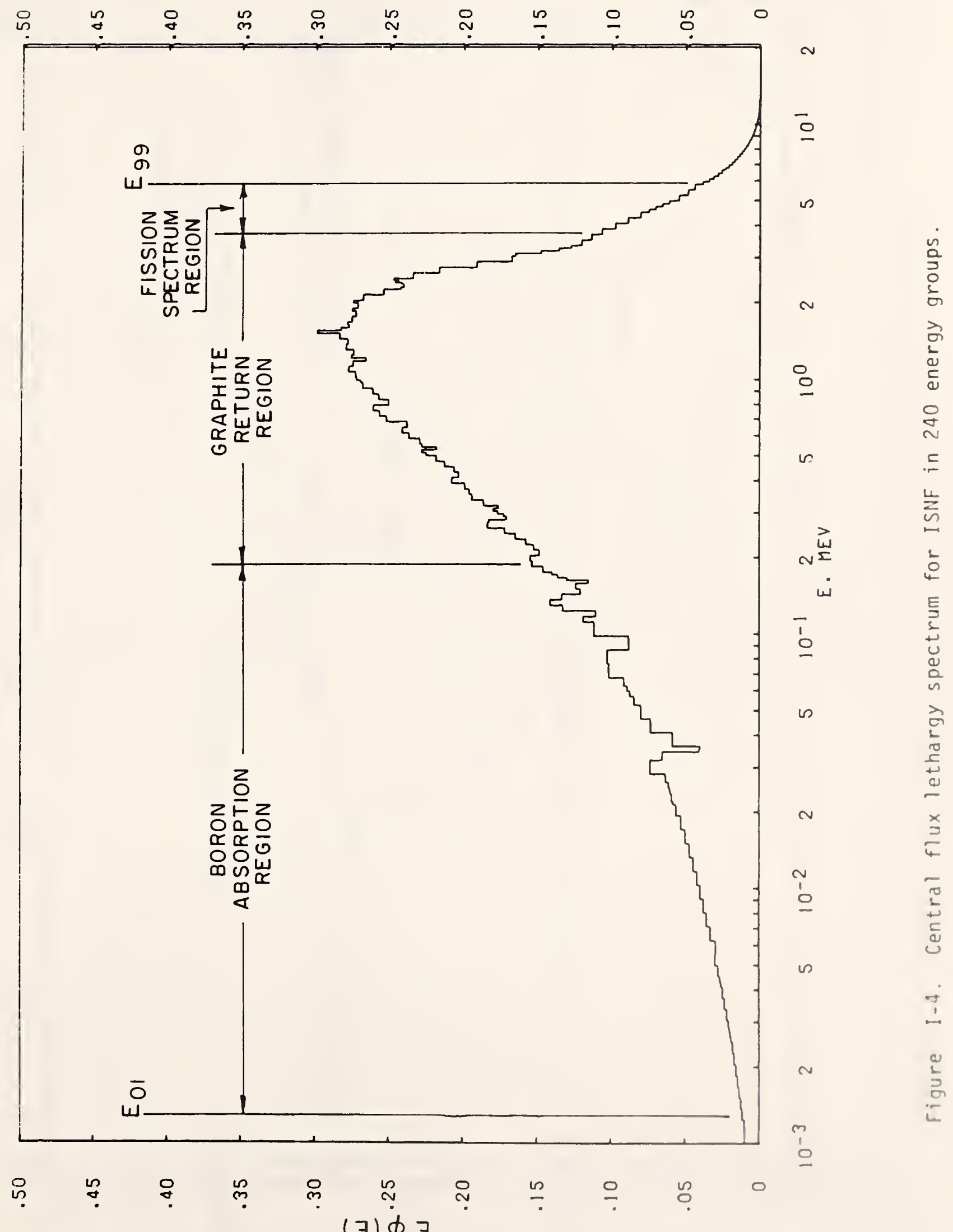




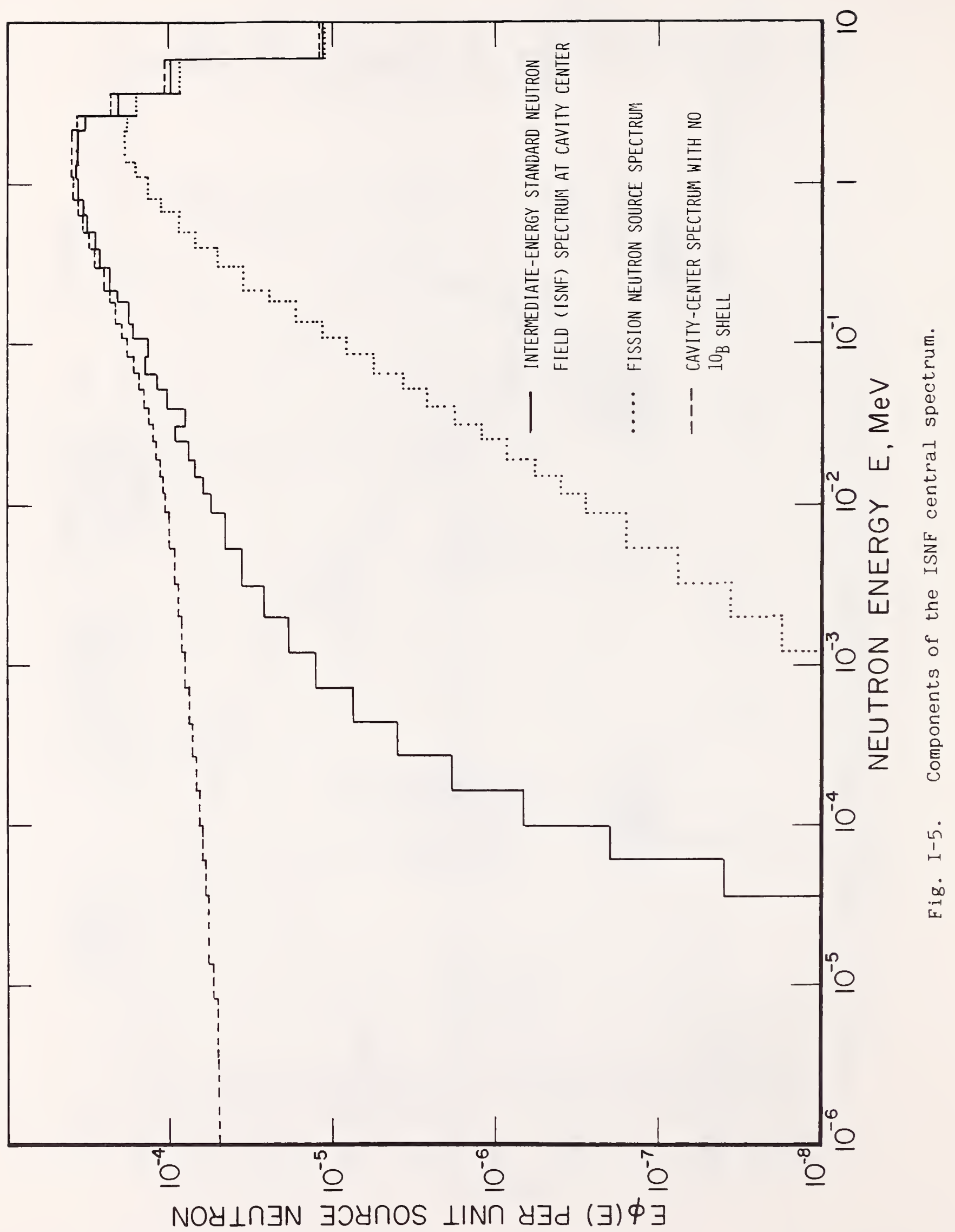




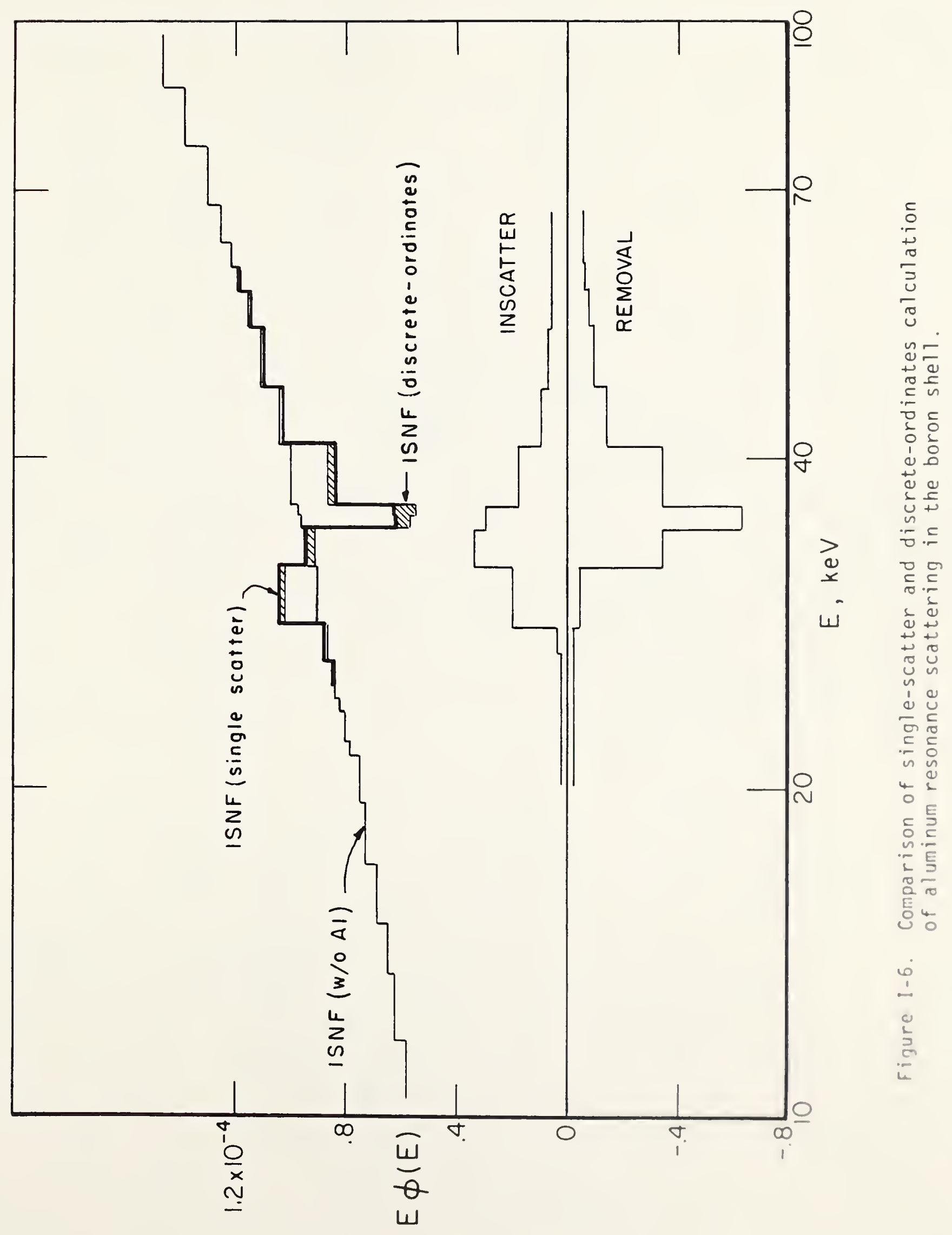





[Ac81a] Acquah, I., Glumac, B., Remec, I., and Najzer, M., "Spectral Indices of Some Threshold Reactions Measured in Uranium-235 Fission Spectrum," Proc. Advisory Group Meeting on Nuclear Data for Radiation Damage Assessment and Related Safety Aspects, IAEA-TECDOC-263, Vienna (Oct. 1981).

[Ad77a] Adamov, V. M., Alexandrov, B. M., Alkhazov, I. D., Drapchinsky, L. V., Kovalenko, S. S., Kostochkin, 0. I., Kudriavzev, G. Yu., Malkin, L.Z., Petrzhak, K. A., Pleskachevsky, L. A., Fomichev, A. V., and Shapakov, V. I., "Absolute $235 \mathrm{U}, 238 \mathrm{U},{ }^{237} \mathrm{~Np}$ Fast Neutron Fission Cross-Section Measurements," Proc. Symposium on Neutron Standards and Applications, March 1977, Washington, D.C., NBS Special Publication 493.

[A1 75a] Alberts, W. G., Bortfeldt, J., Gunther, E., Knauf, K., Matzke, M., Rassl, G., Siegel, V., and Walz, K. F., "Measurement of Cross Sections for Threshold Reaction Induced by ${ }^{252} \mathrm{Cf}$ Spontaneous Fission Neutrons," Proceedings Nuclear Cross Sections and Technology Conference, NBS Special Publication 425, Vol. I, p. 273 (1975).

[A175b] Alberts, W. G., Günther, E., Matzke, M., and Rass1, G., "Measurements of Integral Cross Sections in the $252 \mathrm{Cf}$ Fission Spectrum," Proc. of First ASTM-EURATOM Symposium on Reactor Dosimetry, EUR $5667 \mathrm{e} / \mathrm{f}$, Part II, p. 131, Petten, (September 22-26, 1975).

[A182a] Alberts, W. G., Hollnagel, R., Knauf, K., Matzke, M., and Pebara, W., "Measurements with the Niobium Neutron Fluence Detector at the PTB," Proc. Fourth ASTM-EURATOM Symposium on Reactor Dosimetry, Washington, D.C., NUREG/CP-0029, Vol. 1, 433 (March 1982).

[An83a] 1983 Annual Book of ASTM Standards, Section 12, Vol. 12.02, Nuclear (II), Solar, and Geothermal Energy, Standard Practice E693-79; American Society for Testing and Materials, Philadelphia, PA (1983).

[Br70a] Bresesti, A., Bresesti, M., Rota, A., and Rydin, R., "Threshold Reaction Excitation Functions Intercalibrated in a Pure Fission Spectrum," Nucl. Sci. Eng. 40, 331 (1970).

[Br79a] Broadhead, B. L. and Wagschal, J., "The NBS Intermediate-Energy Standard Neutron Field (ISNF) Revisited," Trans. Am. Nucl. Soc. 33 (November, 1979).

[Br79b] Broadhead, B., Private communication (February, 1979).

[Br80a] Broadhead, B. L. and Wagschal, J. J., "The ISNF: A New CSEWG Dosimetry Benchmark (Computational Problems and Their Solution)," Trans. Am. Nucl. Soc. 35, p. 466 (1980).

[Br81a] Brandon, W. E., Cogburn, C. 0., Culp, R. R., Sallee, W. W., and Williams, J. G., "Wall Return of 252 Cf Neutrons at the SEFOR Calibration Center," Trans. Am. Nucl. Soc. 38, 579 (June, 1981). 
[Br81b] Brandon, W. E., Cogburn, C. 0., Culp, R. R., Sallee, W. W., and Witliams, J. G., "Calibration of Neutron Dosimetry Techniques For Use in Reactor Buildings," Proc. Eighth DOE Workshop on Personnel Neutron Dosimetry, PNL-SA-9950, Louisville, Kentucky (June, 1981).

[Ca84a] Carlson, A. D., "Standard Cross-Section Data," Progress in Nuclear Energy, 13, №. 2/3, 95-102 (1984).

[Cr56a] Cranberg, L., Frye, G., Nereson, N., and Rosen, L., Phys. Rev. 103, 662 (1956); see also, Barnard, E., et al., Nucl. Phys. 71, $228(1965)$; Watt, R., Phys. Rev. 87, 1037 (1952).

[Cu80a] Cullen, D. E., Kucherov, N., and McLaughlin, P. M., "International Reactor Dosimetry File-82," IAEA-NDS-41/R. See al so Strohmaier, B., Tagesen, S., and Vonach, H., Physics Datem-Physics Data 13 (1980).

[Da78a] Davis, M. C., and Knol7, G. F., "Fission Cross Sections of 235U and

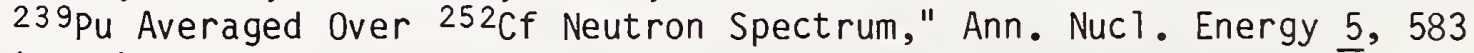
(1978).

[Ei77a] Eisenhauer, C. M. and Grund7, J. A., "Neutron Transport Calculations for the Intermediate-Energy Standard Neutron Field (ISNF) at the National Bureau of Standards," Proceedings International Symposium on Neutron Standards and Applications, NBS Special Publication 493, U. S. Dept. of Commerce, Washington, D.C. (March, 1977).

[EN79a] ENDF/B-V, Report BNL-17541 (ENDF-201), edited by R. Kinsey, available from the National Nuclear Data Center, Brookhaven National Laboratory, Upton, NY (July 1979); See also Magurno, B. A., "ENDF/B-IV Dosimetry Fi 1e," Report BNL-NCS-50446 (1975).

[Fa72a] Fabry, A., "Evaluation of Microscopic Integral Cross Section Averaged in a 235 U Fission Neutron Spectrum," Report BTg 465, CEN/SCK, Mol (1972).

[Fa72b] Fabry, A. and Jenkins, J. D., "Wall Return Neutron Fluxes for High and Intermediate-Energy Cavity Neutron Sources," Trans. Am. Nucl. Soc. 15, 2 (1972).

[Fa74a] Fabry, A. and Czock, K., "Measurements of Integral Cross Section Ratios in Two Dosimetry Benchmark Neutron Fields," INCD(IAEA)-005/G, IAEA/RL/27, IAEA Publ. (1974).

[Fa74b] Fabry, A., "Computational Support to the Development and Characterization of High and Intermediate-Energy Cavity Standard Neutron Sources," Unpublished Report (1974).

[Fa75a] Fabry, A., Ceulemans, H., Vandeplas, P., McElroy, W. N., and Lippincott, E. P., "Reactor Dosimetry Integral Reaction Rate Data," Proceedings of the First International ASTM EURATOM Symposium on Reactor Dosimetry, Proc. EUR 5667 e/f Supplement, p. 23, (September 22-26, 1975). 
[Fa75b] Fabry, A. (CEN/SCK, Mol, Belgium); Grundl, J. A., and Eisenhauer, C. (NBS, USA); "Fundamental Integral Cross Section Measurements in the Thermal-Neutron-Induced Uranium 235 Fission Neutron Spectrum," Proceedings of a Conference on Nuclear Cross Sections and Technology, NBS Special Publications 425, U.S. Dept. of Commerce, Washington, D.C., p. 254 (March, 1975).

[Fa75C] Fabry, A., DeLeeuw, G., and DeLeeuw, S., CEN/SCK, Mol, Belgium, "The Secondary Intermediate-Energy Standard Neutron Field at the Mol- $\Sigma \Sigma$ Facility," Nucl. Tech. 25, 349 (February, 1975).

[Fa78a] Fabry, A., CEN/SCK, Mol, Belgium; McElroy, W. N., Kellogg, L. S., Lippincott, E. P., HEDL; Grund1, J. A., Gilliam, D. M., NBS; and Hansen, G. E., LASL; "Review of Microscopic Intergral Cross Section Data in Fundamental Reactor Dosimetry Benchmark Neutron Fields," Consultants' Meeting on Integral Cross-Section Measurements in Standard Neutron Fields; International Atomic Energy Agency, Vienna, November 15-19, 1976. IAEA 208, Neutron Cross Sections for Reactor Dosimetry (1978).

[Fa79a] Fabry, A., Gilliam, D., Eisenhauer, E., and Slater, W., "The Gold Capture Cross Section in the ISNF," Interlaboratory Reaction Rate Program, 12th Progress Report, HEDL-TME 79-58, (October, 1979).

[Fa82a] Fabry, A., Minsart, G., Cops, F., and Deleeuw, S., "The Mol Cavity Fission Spectrum Standard Neutron Field and Its Applications," Proc. Fourth ASTM-EURATOM Symposium on Reactor Dosimetry, Washington, D.C., NUREG/CP-0029, Vol. 2, 665 (March, 1982).

[Fl77a] Fleming, R., and Spiegel, V., "Measurement of Threshold Reaction Cross Section Ratios in Fission Neutron Fields," Proc. Second ASTM EURATOM Symposium on Reactor Dosimetry, NUREG/CP-0004, Palo Alto, CA (October, 1977).

[Gi75a] Gilliam, D. M., Eisenhauer, C., Heaton II, H. T., and Grundl, J. A., "Fission Cross Section Ratios in the ${ }^{252} \mathrm{Cf}$ Neutron Spectrum ( ${ }^{235} \mathrm{U}$, $\left.{ }^{238} \mathrm{U},{ }^{239} \mathrm{Pu},{ }^{237} \mathrm{~Np}\right)$," Proceedings of a Conference on Nuclear Cross Sections and Technology, Washington, D.C. NBS Special Publication 425, Vol. I, March 1975. p. 270 .

[Gi77a] Gilliam, D. M., "Integral Measurement Results in Standard Fields," Proceedings International Symposium on Neutron Standards and Applications, NBS Special Publication 493, U.S. Dept. of Commerce, Washington, D.C. (March, 1977).

[Gi79a] Gilliam, D. M., Grund1, J., Spiegel, V., Eisenhauer, C., NBS; Maddison, C., and Carpenter, S., ANL; "238U Capture/235U Fission Cross Section in ISNF," Interlaboratory Reaction Rate Program 12th Progress Report, HEDL-TME 79-58 (October, 1979).

[Gi 84a] Gilliam, D. M., Grund1, J., Lamaze, G., and McGarry, E. D., "Cross Section Measurements in the 235 U Fission Spectrum Neutron Field," Proceedings Fifth ASTM-EURATOM Symposium on Reactor Dosimetry, Geesthacht, Germany (September, 1984). 
[Gr62a] Grundl, J., "Cavity Fission Spectra," Trans. Am. Nucl. Soc. $\underline{5}, 381$ (1962).

[Gr70a] Grundl, J. A., "Fission-Neutron Spectra: Macroscopic and Integral Results," Proc. Symposium on Neutron Standards and Flux Normalization, ANL, CONF-701002 (October, 1970).

[Gr72a] Grund1, J. A., "Measurement of the Average Fission Cross-Section $\bar{\sigma}_{f}(238 \mathrm{U})$ for $235 \mathrm{U}$ and $239 \mathrm{Pu}$ Fission Neutrons," IAEA Consultants Meetings on Prompt Fission Neutron Spectra, Vienna, Austria (August 1971). IAEA Proceedings (1972).

[Gr73a] Green, L., et a1., Nucl. Sci. Eng., 50, 257 (1973); see also, Knitter, H., et al., Atomkernenergie, Bd. 22, p. 84 (1973); Meyer, W., et al., Trans. Am. Nucl. Soc., 19, 480 (1974); Jeki, L., et al., Proc. Meeting on Prompt Fission Neutron Spectra, Vienna (1971).

[Gr75a] Green, L., "Absorption Cross Section Measurements for ${ }^{252} \mathrm{Cf}$ Spontaneous Fission Neutron," Nucl. Sci. Eng. 58, 361 (1975).

[Gr75b] Grundl, J. and Eisenhauer, C., "Fission Rate Measurements for Materials Neutron Dosimetry in Reactor Environments," Proceedings First ASTM-EURATOM Symposium on Reactor Dosimetry, EUR5667 e/f, Commission of the European Committees, Petten (September, 1975).

[Gr75c] Grundl, J. A. and Eisenhauer, C. M., "Fission Spectrum Neutrons for Cross Section Validation and Neutron Flux Transfer," Proceedings of a Conference on Nuclear Cross Sections and Technology, NBS Special Publication 425, U.S. Dept. of Commerce, Washington, D.C. (March, 1975).

[Gr76a] Green, N. M., Ford III, W. E., et al., "AMPX: A Modular code System for Generating Coupled Multigroup Neutron-Gamma Libraries from ENDF/B," ORNL/TM-3706 (March, 1976).

[Gr77a] Grund1, J. A., Spiegel, V., Eisenhauer, C. M., Heaton II, H. T., Gilliam, D. M. NBS; and Bigelow, J., ORNL; "A Californium-252 Fission Spectrum Irradiation Facility for Neutron Reaction Rate Measurements," Nucl. Tech. 32, 315 (March, 1977).

[Gr77b] Grund1, J. and Eisenhauer, C., "Fission Reaction Rate Standards and Applications," Proc. Inter. Symp. on Neutron Standards and Applications, NBS Special Publication 493, U.S. Department of Commerce, Washington, D.C. (March, 1977).

[Gr77c] Grund1, J. and Eisenhauer, C. M., "The Intermediate-Energy Standard Neutron Field (ISNF) at the National Bureau of Standards - Design Specifications and Spectrum Calculations," NBS Internal Document (March, 1977).

[Gr78a] Grundl, J. and Eisenhauer, C., "Benchmark Neutron Fields for Reactor Dosimetry," IAEA Consultants Meeting on Neutron Cross Sections for Reactor Dosimetry, Proc: IAEA-208 (1978). 
[Gr82a] Grund1, J. A., Eisenhauer, C. M., and McGarry, E. D., "Neutron Flux Measurements in the Pressure Vessel Cavity of an Operating U.S. Power Reactor," LWR Pressure Vessel Irradiation Surveillance Dosimetry Progress Report, HEDL-Time 8218, NUREG/CR-2805 Vol. I. (March, 1982).

[Gr82b] Grundl, J., Gilliam, D., McGarry, E. D., Eisenhauer, C., and Soran, P., "Revised Value for the ${ }^{252} \mathrm{Cf}$ Fission-Spectrum-Averaged Cross Section for $235 \mathrm{U}$ Fission," private communication to CSEWG (May, 1982).

[Gr83a] Grund1, J., and Gilliam, D., "Fission Cross Section Ratio Measurements in Reactor Physics and Dosimetry Benchmarks," Trans. Am. Nucl. Soc., 44, June (1983).

[Gr85a] Grund1, J., "Examination of ${ }^{10} \mathrm{~B}(\mathrm{n}, \mathrm{He})$ and ${ }^{6} \mathrm{Li}(\mathrm{n}, \mathrm{He})$ Cross Section Measurements in Reactor Physics Benchmarks," Proc. Conf. on Nuclear Data for Basic and Applied Science, Santa Fe, NM (May, 1985).

[Ha76a] Hale, G. M., Stewart, L., and Young, P. C., "Light Element Standard Cross Sections for ENDF/B Version IV," LA6518-MS (October, 1976).

[Ha78a] Hannan, A. and Williams, J. G., "Activation Foil Data for NISUS, Mol- $-\Sigma \Sigma$, and $235 U$ Fission Spectra," IAEA Consultants Meeting on Neutron Cross Sections for Reactor Dosimetry, Vienna (Nov. 1976), IAEA Proceedings (1978).

[He75a] Heaton II, H. T., Grund1, J. A., Spiegel Jr., V., Gilliiam, D. M., and Eisenhauer, C., "Absolute ${ }^{235}$ U Fission Cross Section for ${ }^{252} \mathrm{Cf}$ Spontaneous Fission Neutrons," Proceedings of a Conference on Nuclear Cross Sections and Technology, March 1975, Washington, D.C., NBS Special Publication 425.

[He75b] Heaton II, H. T., Menke, J. L., Schrack, R. A., and Schwartz, R. B., "Total Neutron Cross Section of Carbon from 1 keV to $15 \mathrm{MeV}$," Nucl. Sci. Eng. 56, 27 (1975).

[He76a] Heaton II, H. T., Gilliam, D. M., Spiegel, V., Eisenhauer, C., and Grund1, J. A., "Fission Cross Sections of $235 \mathrm{U}, 238 \mathrm{U}$, and $239 \mathrm{Pu}$ Averaged Over the ${ }^{252} \mathrm{Cf}$ Fission Neutron Spectron," Proceedings of the NEANDC/NEACRP Specialist Meeting on Fast Neutron Fission Cross Sections of U-233, U-235, U-238, and Pu-239, Argonne, Iliinois. ANL76-90, ERDA-NDC-5/L, NEANDC(US)-199/L. p. 333, June 1976 .

[Hi75a] Hill, T. R., "ONETRAN: A Discrete Ordinates Finite Element Code for the Solution of the One-Dimensional Multigroup Transport Equation," LA-5990-MS, Los Alamos Scientific Lab. (June, 1975).

[Is73a] Islam, N. and Knitter, H., Nucl. Sci. Eng. 50, 108 (1973); see also, Johansson, P., et al., Nucl. Data for Reactors (Proc. Conf.), Helsinki (1970); and Ref. [Gr75c] above. 
[Ki78a] Kimura, I., Kobayashi, K., Hayashi, Shu A., Yamamoto, S., Gotoh, H., and Yagi, H., "Measurement and Evaluation of Threshold Reaction Cross Section in Standard Neutron Fields," IAEA Consultants Meeting on Neutron Cross Sections for Reactor Dosimetry, Vienna (Nov. 1976) IAEA Proceedings, Vol. II, p. 265 (1978).

[K080a] Kobayashi, K. and Kimura, I., "Fission Spectrum Averaged Cross Sections with Standard Neutron Fields," Proc. Third ASTM-EURATOM Symp. on Reactor Dosimetry (Ispra, 1979), ISBN 92-825-1903-1; Commission of European Communities, EUR6813, Luxembourg (1980).

[LA78a] LASL Group TD 6, "MCNP-A General Monte Carlo Code for Neutron and Photon Transport," LA-7396-M, Los Alamos Scientific Lab. (July, 1978).

[La80a] LaBauve, R. J., George, D. C., Muir, D. W., Soran, P. D., and Eisenhauer, C. M., "Data Development Work in Support of the National Bureau of Standards ISNF Project," Los Alamos National Lab. Report LA-8638-SR (1980).

[La82a] Lamaze, G. P., McGarry, E.D., and Schima, F. J., "Integral Reaction Rate Measurements in 252Cf and 235U Fission Spectra," Proc. Int. Conf. on Nuclear Data for Science and Technology, Antwerp, Belgium (September, 1982).

[La84a] Lamaze, G. P., Gilliam, D.M., and McGarry, E.D., "Neutron Fluence and Cross Section Measurents for Fast Neutron Dosimetry," Proc. Conf. Nuclear Methods and Environmental and Energy Research, Puerto Rico, (April, 1984).

[Le57a] Leachman, R. B. and Schmitt, H. W., "Cross Section for $238 \mathrm{U}$ Fission by Fission Neutrons," J. Nucl. Energy 4, 38 (1957).

[Li84a] Li Linpei, "Reflection of ${ }^{252}$ Cf Fission Neutrons from a Concrete Floor," Radiation Protection Dosimetry, 5, 237 (1984).

[Ma78a] Mannhart, W., "Integral Cross Section Measurement With Regard to the Low and High Energy Part of the ${ }^{252} \mathrm{Cf}$ Neutron Spectrum," IAEA Consultants Meeting on Neutron Cross Sections for Reactor Dosimetry, Vienna (November 1976), IAEA Proceedings (1978).

[Ma79a] Mannhart, W. and Alberts, W. G., "Measurement and Calculation of Average Activation Cross Section in the Spontaneous Fission Neutron Field of ${ }^{252}$ Cf," Nucl. Sci. Eng. 69, 333 (1979).

[Ma80a] Mannhart, W. and Perey, F., "Covariance Matrices of Cf-252 SpectrumAveraged Cross Sections," Proc. Third ASTM-EURATOM Symp. on Reactor Dosimetry, EUR 6813, Vol. 2, 1016 (1980).

[Ma81a] Mannhart, W., "Progress In Integral Data and Their Accuracy: Average Neutron Cross Sections in the Californium-252 Benchmark Field," Nucl. Sci. Eng. 77, 40 (1981). 
[Ma82a] Mannhart, W., "Average Neutron Cross Sections in the Cf-252 Benchmark Field," Proc. Fourth ASTM-EURATOM Symposium on Reactor Dosimetry, Washington, D.C., NUREG/CP-0029, Vol. 2, 637 (March, 1982).

[Ma82b] Madland, D. G., and Nix, J. R., "New Calculation of Prompt Fission Neutron Spectra and Average Prompt Neutron Multiplicities," Nucl. Sci. Eng. 81, 213-271 (1982).

[Ma83a] Mannhart, W., "Measurement and Evaluation of Integral Data in the Cf-252 Neutron Field," p. 429 in Nuclear Data for Science and Technology," K. H. Bơckhoff, Ed., D. Reidel PubT. Co., Dordrech, Hol1 and (1983).

[Ma84a] Mannhart, W., "Recent Experiments on Cf-252 Spectrum-Averaged Neutron Cross Sections," Proc. Fifth ASTM-EURATOM Symp. on Reactor Dosimetry, Geesthacht, Germany (September, 1984).

[Ma84b] Mannhart, W., "Spectrum-Averaged Neutron Cross Sections Measured in the U-235 Fission-Neutron Field in MOL," Proc. Fifth ASTM-EURATOM Symp. on Reactor Dosimetry, Geesthacht, Germany (September, 1984).

[Ma84c] Madland, D. G., LaBauve, R. J., and Nix, J. R., "Differential and Integral Comparisons of Three Representations of the Prompt Neutron Spectrum for the Spontaneous Fission of ${ }^{252} \mathrm{Cf}$, "LOS Alamos National Lab. Report LA-UR-84-3557 (1984).

[Mc82a] McGarry, E. D., Lamaze, G. P., Eisenhauer, C. M., Gi17iam, D. M., and Schima, F. J., "NBS ISNF and Cavity Fission U-235 Standard Neutron Fields," Proc. Fourth ASTM-EURATOM Symposium on Reactor Dosimetry, Washington, D.C., NUREG/CP-0029, Vol. 2, 597 (March, 1982).

[Mc84a] McGarry, E. D., Eisenhauer, C. M., Gilliam, D. M., Grundl, J., and Lamaze, G. P., "The U.S. U-235 Fission Spectrum Standard Neutron Field Revisited," Proceedings Fifth ASTM-EURATOM Symposium on Reactor Dosimetry, Geesthacht, Germany (September, 1984).

[0182a] 01iver, B. M., Farrar, H., Lippincott, E. P., and Fabry, A., "Spectrum-Integrated Helium Generation Cross Sections for ${ }^{6} \mathrm{Li}$ and ${ }^{10 B}$ in the Sigma Sigma and Fission Cavity Standard Neutron Fields," Proceedings Fourth ASTM-EURATOM Symposium on Reactor Dosimetry, Washington, D.C., NUREG/CP-0029, Vol. 2, 889 (March, 1982).

[0184a] 01 iver, B. M., Farrar, H., Gi17iam, D. M., and Lippincott, E. P., "Spectrum-Integrated Helium-Generation Cross Sections for $6 \mathrm{Li}$ and ${ }^{10 \mathrm{~B}}$ in the Intermediate-Energy Standard Neutron Field," Proceedings Fifth ASTM-EURATOM Symposium on Reactor Dosimetry, Geesthacht, Germany (September, 1984).

[Pa80a] Paulsen, A., Widera, R., Vaninbrouckx, R., and Liskien, H., "CrossSection Measurement for the Reaction $103 R h\left(n, n^{\prime}\right)$," Nucl. Sci. Eng. 76, $331-335$ (1980).

[Sm78a] Smith, A., Holt, R., and Whalen, J., "Neutron Scattering from ${ }^{12} \mathrm{C}$ in the Few-MeV Region," Argonne National Laboratory Report ANL/NDM-43 (September, 1978). 
[Sm79a] Smith A., Guenther, P., Winkler, G., and Mcknight, R., "PromptFission-Neutron Spectra of ${ }^{233} \mathrm{U},{ }^{239} \mathrm{Pu}$, and $240 \mathrm{Pu}$ Relative to that of ${ }^{252}$ Cf," Argonne National Lab. Report ANL/NDM 50 (September, 1979).

[So79a] Soran, P. D., LaBauve, R. J., and George, D. C. (LASL); and Eisenhauer, C. M. (NBS); "Neutronic Analysis of the NBS IntermediateEnergy Standard Neutron Field (ISNF)," Trans. Am. Nucl. Soc. 32 (June, 1979).

[So79b] Soran, P. D. and LaBauve, R. J. (LASL); Eisenhauer, C. M. (NBS); and George, D. C. (LASL), "Neutronic Analysis of the NBS IntermediateEnergy Standard Neutron Field (INSF)," Trans. Am. Nucl. Soc. 32, p. 777 (June, 1979).

[Sp77a] Spiege1, V., Eisenhauer, C. M., and Grund1, J. A. (NBS), and Martin Jr., G. C. (GE), "Reaction Rate Measurements and Integral Cross Sections Using the NBS ${ }^{252}$ Cf Fission Neutron Indoor Irradiation Facility," Proc. Second ASTM EURATOM Symposium on Reactor Dosimetry, NUREG/CP-0004, Palo Alto, CA (October, 1977).

[Sp80a] Spiegel, V., Eisenhauer, C. M., Gilliam, D. M., Grundl, J. A., McGarry, E. D., Schroder, I., Slater, W. E., and Schwartz, R. B., " $235 \mathrm{U}$ Cavity Fission Neutron Field Calibration via the ${ }^{252} \mathrm{Cf}$ Spontaneous Fission Neutron Field," IAEA Consultant's Meeting on Neutron Source Properties, Debrecen, Hungary (March, 1980).

[Wa77a] Wattecamps, E., "Review of $1{ }^{10} B(n, \alpha){ }^{7} L i$ Cross-Section Measurements in the Energy Range from $10 \mathrm{keV}$ to $1 \mathrm{MeV}, "$ Symp. on Neutron Standards and Applications, NBS Special Publication 493 (March, 1977).

[Wa79a] Wagschal, J. J., Maerker, R. E., ORNL; and Gilliam, D. M., NBS; "Detailed Error Analysis of Average Fission Cross-Section Measurements in NBS Standard Neutron Fields," Trans. Am. Nucl. Soc. 33, 823 (November, 1979).

[Wa81a] Wagschal, J. J. and Broadhead, B. L., "Evaluation of the New ISNF OneDimensional Model," Trans. Am. Nucl. Soc. 39, p. 887 (November, 1981).

[We72a] Werle, H. and Bluhm, H., J. Nucl. Energy 26, 165 (1972); see also, Meadows, J., Phys. Rev. 157, 1076 (1967); Conde, H., and During, G., Arkiv for Fysik, 29, 313 (1965); Yu. S. Zamyatin, et al., Nucl. Data for Reactors (Proc. Conf.), Helsinki (1970).

[Wi70a] Wiedling, T., Proc. Symp. on Neutron Standards and Flux Normalization, ANL (1970); see also Smith, A. B., Proc. Consultants Meeting on Prompt Fission Neutron Spectra, Vienna (1971); Koster, A., Ibid., Vienna (1971).

[Wi81a] Winkler, C., Spiegel, V., Eisenhauer, C. M., and Smith, D. L., "Measurement of the Average Activation Cross Section for the Reaction ${ }^{63} \mathrm{Cu}(n, \alpha){ }^{60} \mathrm{Co}$ in the Spontaneous Fission Neutron Field of Californium252," Nuc1. Sci. Eng. 78, (1981). 

